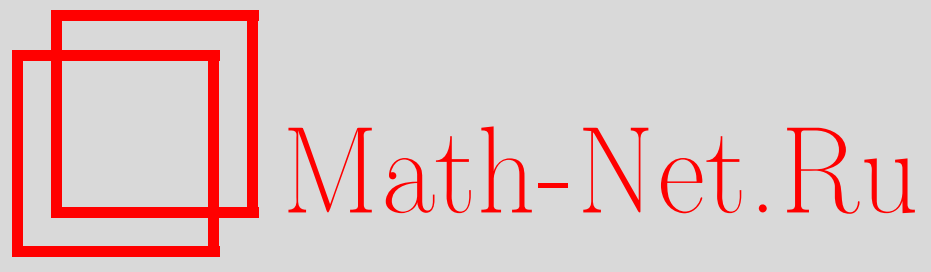

М. З. Гараев, Суммы и произведения множеств и оценки рациональных тригонометрических сумм в полях простого порядка, УМН, 2010, том 65, выпуск 4, 5-66

DOI: https://doi.org/10.4213/rm9367

Использование Общероссийского математического портала Math-Net.Ru подразумевает, что вы прочитали и согласны с пользовательским соглашением http://www . mathnet.ru/rus/agreement

Параметры загрузки:

IP: 3.89 .185 .249

26 апреля 2023 г., 17:05:39

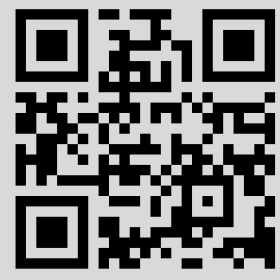


УДК $511+519.1$

\section{Суммы и произведения множеств \\ и оценки рациональных тригонометрических сумм в полях простого порядка}

\section{3. Гараев}

В настоящей работе дается обзор основных результатов по проблеме сумм и произведений множеств в полях простого порядка и их применений к оценкам рациональных тригонометрических сумм.

Библиография: 85 названий.

Ключевые слова: сумма множеств, произведение множеств, поле простого порядка, тригонометрические суммы.

\section{СОДЕРЖАНИЕ}

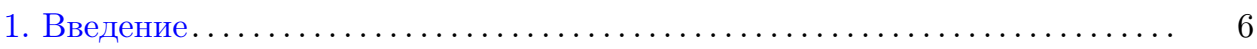

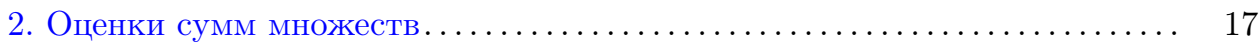

2.1. Неравенство треугольника Ружи ..................... 17

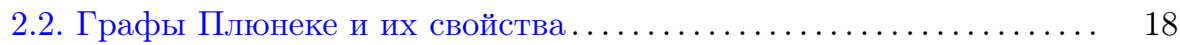

2.3. Неравенство Ружи-Плюнеке ........................... 21

2.4. Оценка типа Балога-Семереди-Гауэрса.................. 22

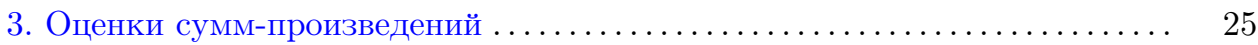

3.1. Доказательство оценки сумм-произведений.............. 27

3.2. Вариант проблемы сумм-произведений с разными множествами 28

4. Применения к тригонометрическим суммам .................. 39

4.1. Формулировка результатов.......................... 39

4.2. Основное утверждение . . . . . . . . . . . . . . . . . . . 41

4.3. Вывод оценок полилинейных тригонометрических сумм ....... 44

4.4. Тригонометрические суммы Морделла .................... 47

4.5. Случай составных модулей........................ 53

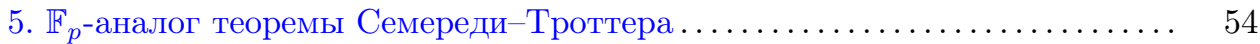

5.1. Аналог теоремы Семереди-Троттера .................... 55

5.2. Растягивающиеся отображения от двух переменных ......... 56

5.3. Оценки некоторых двойных тригонометрических сумм........ 58

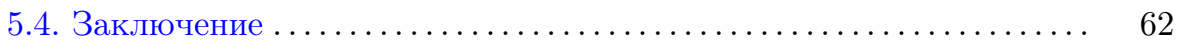

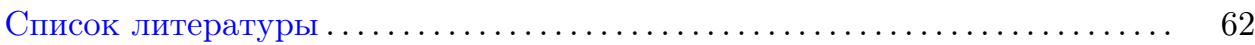

(C) M. 3. ГАРАЕв, 2010 


\section{1. Введение}

На протяжении всей статьи $p$ - большое простое число, $\mathbb{F}_{p}$ - поле классов вычетов по модулю $p$. Каждый класс вычетов часто будем отождествлять с его конкретным представителем. Для конечного множества $X$ через $|X|$ будем обозначать его мощность. Через $\varepsilon$ обозначается маленькая положительная величина.

Оценка сумм-произведений впервые появилась в работе Эрдёша и Семереди [1] для подмножеств кольца целых чисел. Они доказали, что существует положительное число $c$ такое, что для любого конечного непустого множества $A$, состоящего из целых чисел, либо сумма множеств

$$
A+A:=\left\{a_{1}+a_{2}: a_{1} \in A, a_{2} \in A\right\},
$$

либо же произведение множеств

$$
A A:=\left\{a_{1} a_{2}: a_{1} \in A, a_{2} \in A\right\}
$$

имеет мощность не меньшую, чем $|A|^{1+c}$. Если $A$ составляет арифметическую или геометрическую прогрессию, то соответственно будем иметь $|A+A| \leqslant 2|A|$ или $|A A| \leqslant 2|A|$. В частности, результат Эрдёша и Семереди можно интерпретировать как утверждение о несуществовании множества, которое, в некотором смысле, вело бы себя как арифметическая и одновременно как геометрическая прогрессия.

Эрдёш и Семереди выдвинули гипотезу, что для любого положительного числа $\varepsilon>0$ существует $c=c(\varepsilon)>0$ такое, что

$$
\max \{|A+A|,|A A|\} \geqslant c|A|^{2-\varepsilon} .
$$

$\mathrm{K}$ настоящему моменту наилучший результат в этой проблеме принадлежит Шолимоши [2]:

$$
\max \{|A+A|,|A A|\} \geqslant c|A|^{4 / 3-\varepsilon}, \quad c=c(\varepsilon)>0 .
$$

Тао в своей монографии [3] отмечает, что задача о получении аналога результата Эрдёша и Семереди в полях $\mathbb{F}_{p}$ была поставлена в 1999 г. Т. Вольфом (в частной беседе) для подмножеств $A \subset \mathbb{F}_{p}$ мощности порядка $p^{1 / 2}$. Известные к тому моменту методы не проходили для конечных полей, и решение этой задачи требовало новых идей. Вольф был мотивирован связью этой задачи с аналогом проблемы Какея в конечных полях. ${ }^{1}$

Задача сумм-произведений в $\mathbb{F}_{p}$ была решена в работах Бургейна, Глибичука и Конягина [5] и Бургейна, Каца и Тао [6]. Они доказали следующую теорему, которая называется оценкой сумм-произведений в полях простого порядка.

ТЕОРема 1.1. Для любого $\varepsilon>0$ существует $\delta=\delta(\varepsilon)>0$ такое, что если $A \subset \mathbb{F}_{p} u|A|<p^{1-\varepsilon}$, то выполняется оценка ${ }^{2}$

$$
\max \{|A+A|,|A A|\} \geqslant|A|^{1+\delta} .
$$

\footnotetext{
${ }^{1}$ Аналог проблемы Какея в конечных полях недавно был решен Двиром [4] с помощью других идей.

${ }^{2}$ Работа [6] предполагала дополнительное условие $|A|>p^{\varepsilon}$, которое было снято в [5] с помощью аргументов, основанных на методе Степанова [7].
} 
В условии теоремы требование $|A|<p^{1-\varepsilon}$ является естественным, так как если $|A|$ имеет порядок близкий к $p$, то обе величины $|A+A|$ и $|A A|$ будут по порядку близки к $|A|$.

Оценка сумм-произведений и ее варианты нашли много замечательных применений в разных областях математики. С ее помощью Бургейн, Глибичук и Конягин получили не имевшие до этого аналогов оценки рациональных тригонометрических сумм. Прежде чем сформулировать их результаты, напомним некоторые хорошо известные факты из теории.

Пусть $m \geqslant 2$ - целое число. Будем рассматривать рациональные тригонометрические суммы

$$
S:=\sum_{x} e^{2 \pi i x / m}
$$

где $x$ пробегает некоторую систему из $N$ целых чисел. Так как $\left|e^{2 \pi i x / m}\right|=1$, то тривиальная оценка дает $|S| \leqslant N$. Центральной задачей теории является получение оценки вида $|S| \leqslant \Delta N$ с как можно меньшим значением $\Delta=\Delta(N)$. Особенно важными являются те оценки, в которых $\Delta=\Delta(N) \rightarrow 0$ при $N \rightarrow \infty$.

Рациональные тригонометрические суммы и их оценки являются основным инструментом при решении ряда задач теории чисел и имеют приложения во многих разделах математики. Укажем следующую связь между оценками рациональных тригонометрических сумм и аддитивными сравнениями, которую можно найти в ранних работах Виноградова (см. [8]).

Лемма 1.1. Пусть $m \geqslant 2$ - целое число, и пусть числа и и $v$, независимо друг от друга, пробегают систему цельх значений

$$
u=u_{1}, \ldots, u_{N} ; \quad v=v_{1}, \ldots, v_{M},
$$

причем

$$
\left|\sum_{u} e^{2 \pi i a u / m}\right| \leqslant R
$$

для любого иелого числа а, не делящегося на $m, u$

$$
\sum_{a=1}^{m-1}\left|\sum_{v} e^{2 \pi i a v / m}\right| \leqslant D
$$

Тогда число Т решений сравнения

$$
u_{x} \equiv v_{y}(\bmod m), \quad 1 \leqslant x \leqslant N, \quad 1 \leqslant y \leqslant M,
$$

можно представить в виде

$$
T=\frac{N M}{m}+\theta \frac{R D}{m}, \quad|\theta| \leqslant 1 .
$$

Действительно, так как

$$
\frac{1}{m} \sum_{a=0}^{m-1} e^{2 \pi i a(u-v) / m}= \begin{cases}1, & \text { если } u \equiv v(\bmod m), \\ 0, & \text { если } u \neq \equiv(\bmod m),\end{cases}
$$


то имеет место равенство

$$
T=\sum_{u} \sum_{v} \frac{1}{m} \sum_{a=0}^{m-1} e^{2 \pi i a(u-v) / m}=\frac{1}{m} \sum_{a=0}^{m-1} \sum_{u} \sum_{v} e^{2 \pi i a(u-v) / m} .
$$

Выделяя член, соответствующий значению $a=0$, получим

$$
\left|T-\frac{N M}{m}\right| \leqslant \frac{1}{m} \sum_{a=1}^{m-1}\left|\sum_{u} e^{2 \pi i a u / m}\right|\left|\sum_{v} e^{2 \pi i a v / m}\right| \leqslant \frac{R D}{m},
$$

откуда следует утверждение леммы.

Таким образом, имея нужные оценки тригонометрических сумм, можно выводить асимптотические формулы для числа решений соответствующих аддитивных сравнений. Одной из простейших оценок рациональных тригонометрических сумм является оценка билинейной суммы (рассмотрим только случай простого модуля $p$ ), утверждающая, что для любого целого числа $a$ такого, что $a \not \equiv 0(\bmod p)$, и для любых непустых подмножеств $X, Y$ поля $\mathbb{F}_{p}$ имеет место оценка

$$
W:=\sum_{x \in X}\left|\sum_{y \in Y} e^{2 \pi i a x y / p}\right| \leqslant(p|X||Y|)^{1 / 2} .
$$

Действительно, применив неравенство Коши-Буняковского к сумме по переменной $x \in X$, затем распространив суммирование по $x$ до полной системы вычетов, получим

$$
W^{2} \leqslant|X| \sum_{x=0}^{p-1}\left|\sum_{y \in Y} e^{2 \pi i a x y / p}\right|^{2}=|X| \sum_{y_{1} \in Y} \sum_{y_{2} \in Y} \sum_{x=0}^{p-1} e^{2 \pi i a x\left(y_{1}-y_{2}\right) / p}=p|X||Y|,
$$

откуда следует оценка (1).

Оценка (1) содержится в учебнике Виноградова [9] в качестве упражнения (даже в более общей форме, см. ниже лемму 4.1). Несмотря на простоту доказательства, она имеет многочисленные применения в изучении широкого класса аддитивных сравнений. Из этой оценки видно, что если величины $|X|$ и $|Y|$ по порядку не меньше, чем $p^{1 / 2+\varepsilon}$, то $W$ оценивается нетривиально.

В качестве простого применения вышеописанной техники, установим следующее предложение, из которого следует оценка сумм-произведений для множеств $A$ сравнительно большой мощности.

ПреДЛОЖеНИЕ 1.1. Для любого непустого множества $A \subset \mathbb{F}_{p}$ имеет место оценка

$$
\max \{|A+A|,|A A|\}>c \min \left\{\frac{|A|^{2}}{p^{1 / 2}}, p^{1 / 2}|A|^{1 / 2}\right\},
$$

где с - положительная абсолютная константа.

Оценка (2) получена в работе [10] и улучшает некоторые результаты работ [11] и [12]. Из нее следует утверждение теоремы 1.1 в случае $|A|>p^{1 / 2+\varepsilon}$. Кроме того, при $|A|>p^{2 / 3}$ из оценки (2) следует, что

$$
\max \{|A+A|,|A A|\}>c p^{1 / 2}|A|^{1 / 2} .
$$


Интересно, что последняя оценка в общей формулировке является наилучшей с точностью до постоянного множителя. Действительно, пусть $g$ - первообразный корень по модулю $p$. Из принципа Дирихле следует, что для любых целых чисел $N \in[1, p]$ и $M \approx p^{1 / 2} N^{1 / 2}$ существует целое число $L$ такое, что

$$
\left|\left\{g^{x}: 1 \leqslant x \leqslant M\right\} \cap\{\{L+1, L+2, \ldots, L+M\}(\bmod p)\}\right|>\frac{M^{2}}{2 p} \approx N
$$

(здесь $x \approx y$ означает, что для некоторых положительных констант $c_{1}, c_{2}$ выполняется $\left.c_{1} x \leqslant y \leqslant c_{2} x\right)$. Следовательно, если

$$
A \subset\left\{g^{x}: 1 \leqslant x \leqslant M\right\} \cap\{\{L+1, L+2, \ldots, L+M\}(\bmod p)\}, \quad|A| \approx N,
$$

то $\max \{|A+A|,|A A|\} \leqslant 2 M \approx p^{1 / 2}|A|^{1 / 2}$. Отсюда следует, что если $N \in[1, p]$, то существует множество $A \subset \mathbb{F}_{p}$ такое, что $|A|=N$ и в то же время

$$
\max \{|A+A|,|A A|\}<c p^{1 / 2}|A|^{1 / 2} .
$$

Это наблюдение свидетельствует о точности оценки (2) для подмножеств $A \subset \mathbb{F}_{p}$ мощности $|A|>p^{2 / 3}$.

Для доказательства предложения 1.1 можно предположить, что $\{0\} \notin A$. Рассмотрим в поле $\mathbb{F}_{p}$ уравнение

$$
x a_{1}^{-1}=y-a_{2}, \quad\left(x, a_{1}, a_{2}, y\right) \in(A A) \times A \times A \times(A+A) .
$$

Для любого упорядоченного набора $\left(a_{1}, a_{2}, a_{3}\right) \in A \times A \times A$ набор

$$
\left(a_{1} a_{3}, a_{1}, a_{2}, a_{3}+a_{2}\right) \in(A A) \times A \times A \times(A+A)
$$

является решением уравнения (3). Разным наборам $\left(a_{1}, a_{2}, a_{3}\right) \in A \times A \times A$ соответствуют разные наборы $\left(a_{1} a_{3}, a_{1}, a_{2}, a_{3}+a_{2}\right)$. Следовательно, число $T$ решений уравнения (3) удовлетворяет условию $T \geqslant|A|^{3}$. Теперь оценим $T$ сверху с помощью тригонометрических сумм. Из оценки $(1)$ при $n \not \equiv 0(\bmod p)$ следует, что

$$
\left|\sum_{x \in A A} \sum_{a_{1} \in A} e^{2 \pi i n x a_{1}^{-1} / p}\right| \leqslant \sqrt{p|A A||A|} .
$$

Кроме того, с помощью неравенства Коши-Буняковского получаем

$$
\begin{aligned}
& \sum_{n=0}^{p-1}\left|\sum_{a_{2} \in A} e^{2 \pi i n a_{2} / p}\right|\left|\sum_{y \in A+A} e^{2 \pi i n y / p}\right| \\
& \quad \leqslant\left(\sum_{n=0}^{p-1}\left|\sum_{a_{2} \in A} e^{2 \pi i n a_{2} / p}\right|^{2}\right)^{1 / 2}\left(\sum_{n=0}^{p-1}\left|\sum_{y \in A+A} e^{2 \pi i n y / p}\right|^{2}\right)^{1 / 2}=p \sqrt{|A||A+A|} .
\end{aligned}
$$

Поэтому применение леммы 1.1 с нужным выбором системы чисел $u$ и $v$ влечет, что

$$
T \leqslant \frac{|A A||A|^{2}|A+A|}{p}+\sqrt{p|A A||A|} \sqrt{|A||A+A|} .
$$

Так как $T \geqslant|A|^{3}$, то для $\max \{|A+A|,|A A|\}$ получаем требуемую оценку. 
Одной из важнейших тригонометрических сумм в теории чисел является сумма Гаусса

$$
S_{n}(a, m)=\sum_{x=0}^{m-1} e^{2 \pi i a x^{n} / m}, \quad(a, m)=1 .
$$

С ее основными свойствами можно ознакомиться в монографиях [13], [14]. Из результатов Гаусса известно, что

$$
\left|S_{2}(a, m)\right|= \begin{cases}\sqrt{m} & \text { при } m \equiv 1(\bmod 2) ; \\ \sqrt{2 m} & \text { при } m \equiv 0(\bmod 4) ; \\ 0 & \text { при } m \equiv 2(\bmod 4) .\end{cases}
$$

При изучении сумм Гаусса центральным является случай, когда $m=p$ простое число, так как многие принципиальные вопросы, связанные с суммами Гаусса, сводятся к этому случаю. Нетрудно видеть, что если $d=(n, p-1)$, то $S_{n}(a, p)=S_{d}(a, p)$. Поэтому при оценке суммы $S_{n}(a, p)$ можно предположить, что $n$ является делителем числа $p-1$. Если $x$ пробегает полную систему вычетов по модулю $p$, то для любого $\xi \not \equiv 0(\bmod p)$ система $\xi x$ также пробегает полную систему вычетов по модулю $p$. Поэтому

$$
S_{n}(a, p)=\sum_{x=0}^{p-1} e^{2 \pi i a \xi^{n} x^{n} / p}
$$

Пусть $g$ - первообразный корень по модулю $p$. Так как числа $g^{0}, g^{n}, \ldots, g^{(t-1) n}$, где $t=(p-1) / n$, принадлежат разным ненулевым классам вычетов по модулю $p$, то

$$
t\left|S_{n}(a, p)\right|^{2}=\sum_{\ell=0}^{t-1}\left|\sum_{x=0}^{p-1} e^{2 \pi i a g^{\ell n} x^{n} / p}\right|^{2} \leqslant \sum_{u=0}^{p-1}\left|\sum_{x=0}^{p-1} e^{2 \pi i a u x^{n} / p}\right|^{2}-p^{2}=p J-p^{2},
$$

где $J$ - число решений сравнения

$$
x^{n} \equiv y^{n}(\bmod p), \quad 0 \leqslant x, y \leqslant p-1 .
$$

Если в этом сравнении $y=0$, то $x=0$. Если же $y$ зафиксировать из промежутка $1 \leqslant y \leqslant p-1$, то для $x$ будем иметь не более $n$ возможностей (так как $n$ является делителем числа $p-1$, то на самом деле для $x$ будем иметь в точности $n$ возможностей). Таким образом,

$$
J \leqslant 1+n(p-1) .
$$

Поэтому

$$
\left|S_{n}(a, p)\right| \leqslant \sqrt{n(n-1) p} \leqslant n p^{1 / 2} .
$$

А если еще учитывать равенство

$$
\sum_{u=0}^{p-1} \sum_{x=0}^{p-1} e^{2 \pi i a u x^{n} / p}=p
$$


то можно прийти к классической оценке Харди-Литтлвуда [15] тригонометрических сумм Гаусса: ${ }^{3}$

$$
\left|S_{n}(a, p)\right| \leqslant(n-1) p^{1 / 2} .
$$

Эта оценка является нетривиальной при $n<p^{1 / 2}$. Проблема получения нетривиальной оценки при больших значениях $n$ была темой многих исследований. Так, используя результаты работы [16], Шпарлинский [17] установил оценку

$$
\left|S_{n}(a, p)\right| \ll n^{7 / 12} p^{2 / 3}
$$

являющуюся нетривиальной при $n$ порядка $o\left(p^{4 / 7}\right)$ (здесь и далее будем пользоваться известным обозначением Виноградова: $x \ll y$ означает, что $|x| \leqslant c y$ для некоторой абсолютной константы $c$ ).

Оценка Шпарлинского была улучшена в работе Хиз-Брауна и Конягина [18] на основе метода Степанова [7] (как отмечено в [18], метод работы [16] также близок к методу Степанова). Они доказали оценку

$$
\left|S_{n}(a, p)\right| \ll \min \left\{n^{5 / 8} p^{5 / 8}, n^{3 / 8} p^{3 / 4}\right\},
$$

которая является нетривиальной при $n$ порядка $o\left(p^{2 / 3}\right)$. Этот результат далее был улучшен Конягиным [19], который получил нетривиальную оценку при $n \leqslant p^{3 / 4-\varepsilon}$ (тоже на основе метода Степанова).

Заметим, что множество

$$
H:=\left\{x^{n}(\bmod p): 1 \leqslant x \leqslant p-1\right\}
$$

является подгруппой ${ }^{4}$ мультипликативной группы $\mathbb{F}_{p}^{*}$ порядка $(p-1) / n$. При этом каждый элемент $h \in H$ ровно $n$ способами представляется в виде

$$
h \equiv x^{n}(\bmod p), \quad 1 \leqslant x \leqslant p-1 .
$$

Поэтому сумму Гаусса можно представить в виде

$$
S_{n}(a, p)=1+n \sum_{h \in H} e^{2 \pi i a h / p} .
$$

Следовательно, задача получения нетривиальной оценки для $S_{n}(a, p)$ эквивалентна задаче получения нетривиальной оценки для суммы

$$
S(a ; H):=\sum_{x \in H} e^{2 \pi i a x / p} .
$$

Упомянутая оценка Конягина, таким образом, означает нетривиальную оценку для $S(a ; H)$ при $|H|>p^{1 / 4+\varepsilon}$.

\footnotetext{
${ }^{3}$ Еще один способ вывода этой оценки основан на представлении

$$
S_{n}(a, p)=\sum_{b=1}^{n-1} \sum_{t=1}^{p-1} e^{2 \pi i\left(a g^{t} / p+b t / n\right)} .
$$

Внутренняя сумма по $t$ также называется суммой Гаусса и по абсолютной величине равна $p^{1 / 2}$.

${ }^{4}$ Ввиду цикличности группы $\mathbb{F}_{p}^{*}$, любая подгруппа $\mathbb{F}_{p}^{*}$ совпадает с $H$ при некотором $n$.
} 
Далее, сумму $S(a ; H)$ можно представить в виде полилинейной суммы

$$
S(a ; H)=\frac{1}{|H|^{k-1}} \sum_{x_{1} \in H} \cdots \sum_{x_{k} \in H} e^{2 \pi i a x_{1} \cdots x_{k} / p} .
$$

В этом равенстве можно взять $k=2$ и получить представление $S(a ; H)$ в виде билинейной тригонометрической суммы, к которой можно применить неравенство (1). Однако это неравенство не позволяет получить для $S(a ; H)$ нетривиальную оценку в случае, когда подгруппа $H$ имеет мощность $|H|<p^{1 / 2}$.

Используя оценку билинейной тригонометрической суммы, мы установили оценку сумм-произведений для подмножеств поля $\mathbb{F}_{p}$ сравнительно больших мощностей. Несравненно более значимым является то, что, используя оценки сумм-произведений для подмножеств поля $\mathbb{F}_{p}$ небольших мощностей, удается получить неизвестные ранее оценки полилинейных тригонометрических сумм, которые имеют важные приложения к суммам Гаусса. Первый такой результат был получен в работе Бургейна, Глибичука и Конягина [5]. Они доказали следующую теорему.

ТеОРема 1.2. Для любого $\varepsilon>0$ существуют $\delta=\delta(\varepsilon)>0$ и натуральное число $k=k(\varepsilon)$ такие, что если $X \subset \mathbb{F}_{p} u|X|>p^{\varepsilon}$, то

$$
\max _{(a, p)=1}\left|\sum_{x_{1} \in X} \cdots \sum_{x_{k} \in X} e^{2 \pi i a x_{1} \cdots x_{k} / p}\right|<|X|^{k} p^{-\delta} .
$$

Из теоремы 1.2 , в частности, следует, что если $H$ является подгруппой мультипликативной группы $\mathbb{F}_{p}^{*}$ порядка $|H|>p^{\varepsilon}$, то при $(a, p)=1$ имеет место неравенство

$$
|S(a ; H)|<|H|^{1-\delta}, \quad \delta=\delta(\varepsilon)>0 .
$$

Иначе говоря, для любой сколь угодно малой фиксированной положительной константы $\varepsilon>0$, если $n<p^{1-\varepsilon}$, то сумма Гаусса $S_{n}(a, p)$ допускает нетривиальную оценку.

С момента появления оценки сумм-произведений эта тема стала объектом интенсивных исследований, приведших к новым результатам и приложениям. Недавно Бургейн [20] доказал, что для любого фиксированного числа $k$ и для любого $\varepsilon>0$ существует $\delta=\delta(\varepsilon)>0$ такое, что если $|X|>p^{1 / k+\varepsilon}$, то выполняется неравенство (4). Для сумм Гаусса Бургейн доказал, что существует такая константа $c$, что если $H$ является подгруппой мультипликативной группы $\mathbb{F}_{p}^{*}$ порядка $|H|>e^{c \log p / \log \log p}$, то при $p \rightarrow \infty$ имеет место оценка $^{5}$

$$
\max _{(a, p)=1}|S(a ; H)|=o(|H|) .
$$

Этот результат Бургейна (так же как и вышеприведенный результат Бургейна, Глибичука и Конягина) будет доказан в разделе 4.

$\mathrm{K}$ настоящему моменту неизвестно, можно ли условие $|H|>e^{c \log p / \log \log p}$ заменить на более слабое $|H|>e^{f(p)}$ с некоторой функцией

$$
f(p)=o(\log p / \log \log p)
$$

\footnotetext{
${ }^{5}$ Из этой оценки следует свойство равномерного распределения подгруппы $H$ в поле $\mathbb{F}_{p}$.
} 
так, чтобы оценка (5) оставалась справедливой. Однако известно, что если с ростом $p$ величина $|H|$ растет слишком медленно, то оценка (5) не будет иметь места. Так, нижние оценки для $S(a, H)$ при $|H|$ намного меньших, чем $\log p$, имеются в работе Карацубы [21]. А из результатов Монтгомери [22] (или же см. [23]) по проблеме Турана следует, что для любой константы $B>0$, если $|H|<B \log p$, то

$$
\max _{(a, p)=1}|S(a ; H)|>c|H|, \quad c=c(B)>0 .
$$

Действительно, пусть $|H|=t<B \log p$. Для любого целого числа $2 \leqslant m \leqslant t / 2$ величина

$$
T_{m}:=\frac{1}{p} \sum_{a=0}^{p-1}\left|\sum_{x \in H} e^{2 \pi i a x / p}\right|^{2 m}
$$

равна числу решений уравнения

$$
x_{1}+\cdots+x_{m}=x_{m+1}+\cdots+x_{2 m}, \quad x_{1}, \ldots, x_{2 m} \in H .
$$

Далее, существует $t(t-1) \cdots(t-m+1)$ различных упорядоченных наборов $\left(x_{1}, \ldots, x_{m}\right) \in H^{m}$ с попарно различными координатами. Для каждого такого набора существует $m$ ! различных упорядоченных наборов $\left(x_{m+1}, \ldots, x_{2 m}\right)$, получающихся из набора $\left(x_{1}, \ldots, x_{m}\right)$ перестановкой координат. Следовательно, $T_{m} \geqslant t(t-1) \cdots(t-m+1) m ! \geqslant(t m / 6)^{m}$. Поэтому для достаточно большой константы $C=C(B)$, если $m=[t / C]$, то

$$
\frac{1}{p} \sum_{a=1}^{p-1}\left|\sum_{x \in H} e^{2 \pi a i x / p}\right|^{2 m}=T_{m}-\frac{t^{2 m}}{p}>\left(\frac{t m}{6}\right)^{m}-\frac{t^{2 m}}{p}>\frac{1}{2}\left(\frac{t m}{6}\right)^{m}>\left(\frac{t}{10 C}\right)^{2 m} .
$$

Отсюда следует искомая оценка $\max _{(a, p)=1}|S(a ; H)|>(10 C)^{-1}|H|$.

Таким образом, для того чтобы оценка (5) имела место, необходимо, чтобы выполнялось условие $|H| / \log p \rightarrow \infty$ при $p \rightarrow \infty$. С другой стороны, можно ожидать, что это условие является достаточным. Это вытекало бы в случае справедливости очень смелой гипотезы Монтгомери, Вона и Вули [24]. Из справедливости же гипотезы Конягина [25] вытекало бы, что если для некоторой фиксированной положительной константы $\varepsilon$ выполняется условие $|H|>(\log p)^{1+\varepsilon}$, то имеет место оценка (5).

Рассмотрим теперь тригонометрические суммы со степенями первообразного корня $g$ по модулю $p$. Хорошо известно, что при $N<p$ имеет место оценка

$$
\max _{(a, p)=1}\left|\sum_{x=1}^{N} e^{2 \pi i a g^{x} / p}\right| \ll p^{1 / 2} \log p,
$$

которая является нетривиальной, если $N\left(p^{1 / 2} \log p\right)^{-1} \rightarrow \infty$ при $p \rightarrow \infty$. На самом деле легко показать, что если $N p^{-1 / 2} \rightarrow \infty$ при $p \rightarrow \infty$, то имеет место нетривиальная оценка

$$
\max _{(a, p)=1}\left|\sum_{x=1}^{N} e^{2 \pi i a g^{x} / p}\right|=o(N)
$$


Действительно, пусть $M=\left[N^{1 / 2} p^{1 / 4}\right]$ (таким образом, $M=o(N)$ и $M p^{-1 / 2} \rightarrow \infty$ при $p \rightarrow \infty)$. Для любого целого $1 \leqslant y \leqslant M$ имеем

$$
\sum_{x=1}^{N} e^{2 \pi i a g^{x} / p}=\sum_{x=1}^{N} e^{2 \pi i a g^{x+y} / p}+2 \theta M, \quad|\theta| \leqslant 1 .
$$

Суммируя по множеству $1 \leqslant y \leqslant M$, получим

$$
\sum_{x=1}^{N} e^{2 \pi i a g^{x} / p}=\frac{1}{M} \sum_{x=1}^{N} \sum_{y=1}^{M} e^{2 \pi i a g^{x} g^{y} / p}+o(N) .
$$

Так как двойная сумма в правой части по абсолютной величине, согласно (1), не превосходит $\sqrt{p N M}$, то отсюда получаем оценку (6).

Из результата Бургейна, Глибичука и Конягина (теорема 1.2) легко следует, что для любой сколь угодно малой фиксированной положительной константы $\varepsilon>0$ нетривиальная оценка (6) имеет место при $N>p^{\varepsilon}$. Из упомянутой работы Бургейна [20] следует, что эта оценка справедлива и при $N>e^{c \log p / \log \log p}$ для некоторой абсолютной константы $c>0$ (см. раздел 4, следствие 4.2). Так же как и в случае тригонометрической суммы по подгруппе $H$, для того, чтобы оценка (6) имела место, необходимо, чтобы $N / \log p \rightarrow \infty$ при $p \rightarrow \infty$.

Из теоремы 1.1 можно вывести различные аддитивные свойства произведений множеств. Например, если $H$ является подгруппой мультипликативной группы $\mathbb{F}_{p}^{*}$ порядка $|H|>p^{\varepsilon}$, то из теоремы 1.1 следует, что существует натуральное число $k=k(\varepsilon)$ такое, что любой класс вычетов $\lambda \in \mathbb{F}_{p}$ представляется в виде

$$
x_{1}+\cdots+x_{k}=\lambda
$$

для некоторых $x_{1}, \ldots, x_{k} \in H$. Приведенная в теореме 1.2 оценка тригонометрических сумм позволяет не только получить разрешимость уравнения (7), но также вывести асимптотическую формулу для количества решений этого уравнения.

Конечно же, в отличие от $H$, множество $G:=\left\{g^{x}: 1 \leqslant x \leqslant N\right\}$ не является группой по умножению. Однако при $n=N^{o(1)}$ множество $G$ содержит в себе $n$-кратное произведение множества $G_{1}=\left\{g^{x}: 1 \leqslant x \leqslant N / n\right\}$ мощности $\left|G_{1}\right| \approx$ $N / n=N^{1+o(1)}$. Поэтому из теоремы 1.1 также без труда следует, что для любого $\varepsilon>0$ существует натуральное число $k=k(\varepsilon)$ такое, что любой класс вычетов $\lambda \in \mathbb{F}_{p}$ представляется в виде

$$
g^{x_{1}}+\cdots+g^{x_{k}}=\lambda
$$

для некоторых $1 \leqslant x_{1}, \ldots, x_{k} \leqslant p^{\varepsilon}$. С помощью теоремы 1.2 удается также вывести асимптотическую формулу для количества таких представлений.

Следует отметить, что сильный результат по аддитивным свойствам произведений множеств получен в работе Глибичука и Конягина [26]. Применяя комбинаторные аргументы, Глибичук [27] доказал, что если $A, B \subset \mathbb{F}_{p}$ и $|A||B|>2 p$, то

$$
8 A B:=\left\{a_{1} b_{1}+\cdots+a_{8} b_{8}: a_{i} \in A, b_{i} \in B\right\}=\mathbb{F}_{p} .
$$


Результат работы [26] основан на варианте рассуждений Тао и Ву [28], что для любых множеств $A, B \subset \mathbb{F}_{p}$ с $|B| \geqslant 2$ либо имеет место

$$
\frac{A-A}{B-B}:=\left\{\frac{a_{1}-a_{2}}{b_{1}-b_{2}}: a_{1}, a_{2} \in A, b_{1}, b_{2} \in B, b_{1} \neq b_{2}\right\}=\mathbb{F}_{p},
$$

либо же для любого $\xi \in \mathbb{F}_{p}^{*}$ имеем

$$
\frac{A-A}{B-B}+\xi \not \subset \frac{A-A}{B-B} .
$$

Образец применения этого наблюдения читатель найдет в последующих разделах (см., например, доказательства лемм 3.2 и 3.3).

$\mathrm{K}$ настоящему моменту имеются сравнительно более простые доказательства оценки сумм-произведений, которые также позволяют получать некоторые удовлетворительные явные оценки. В работе [12] в самом важном случае $|A|<p^{1 / 2}$ была получена оценка

$$
|A+A|+|A A| \gg|A|^{15 / 14-\varepsilon} .
$$

Экспонента 15/14 была улучшена Кацем и Шеном [29] до 14/13, а в [30] они получили явную оценку сумм-произведений для подмножеств произвольных конечных полей (т. е. полей не обязательно простого порядка). В работе [31] получен специальный вариант оценки сумм-произведений с привлечением множества $A-A$. В частности, при $|A|<p^{1 / 2}$ была получена оценка

$$
|A-A|+|A A| \gg|A|^{13 / 12-\varepsilon} .
$$

Явные варианты теоремы 1.1 приводят к явным оценкам тригонометрических сумм. В работе [31] было доказано, что для любых подмножеств $X, Y, Z$ множества $\mathbb{F}_{p}^{*}$ имеет место оценка

$$
\left|\sum_{x \in X} \sum_{y \in Y} \sum_{z \in Z} e^{2 \pi i x y z / p}\right|<(|X||Y||Z|)^{13 / 16} p^{5 / 18}(\log p)^{O(1)} .
$$

Если $|X|=|Y|=|Z|=N \approx p^{1 / 2}$, то отсюда получим

$$
\left|\sum_{x \in X} \sum_{y \in Y} \sum_{z \in Z} e^{2 \pi i x y z / p}\right|<N^{3-1 / 144+o(1)} .
$$

Отметим, что из работы Бургейна [20] известно, что нетривиальная оценка со степенным понижением имеет место при оптимальном условии

$$
|X||Y||Z|>p^{1+\varepsilon}
$$

Приведем еще одну явную оценку из работы [31]. Было доказано, что если $g$ является первообразным корнем по модулю $p$ и если $1 \leqslant N<p^{1 / 2}$, то число решений сравнения

$$
g^{x}+g^{y} \equiv g^{z}+g^{t}(\bmod p), \quad 1 \leqslant x, y, z, t \leqslant N,
$$


по порядку не превосходит величины $N^{3-1 / 24+o(1)}$. Отсюда с помощью стандартных методов выводится, что при $N \approx p^{1 / 2}$ имеет место оценка

$$
\max _{(a, p)=1}\left|\sum_{x \leqslant N} e^{2 \pi i a g^{x} / p}\right|<N^{215 / 217+o(1)} .
$$

Присутствие сумм и произведений множеств в полилинейных тригонометрических суммах можно нащупать следующим образом. Для простоты рассмотрим трилинейную сумму

$$
S_{3}=\sum_{x \in X} \sum_{y \in X} \sum_{z \in X} e^{2 \pi i x y z / p} .
$$

В этой сумме уже присутствуют элементы произведения множеств

$$
X X=\{x y: x \in X, y \in X\} .
$$

Если модуль этой суммы возвести в квадрат и воспользоваться неравенством Коши-Буняковского по переменным $y, z$, то получим

$$
\left|S_{3}\right|^{2} \leqslant|X|^{2} \sum_{y \in X} \sum_{z \in X}\left|\sum_{x_{1} \in X} \sum_{x_{2} \in X} e^{2 \pi i\left(x_{1}+x_{2}\right) y z}\right| .
$$

В последнем выражении присутствуют уже элементы суммы множеств

$$
X+X=\left\{x_{1}+x_{2}: x_{1} \in X, x_{2} \in X\right\} .
$$

Так как, согласно оценке сумм-произведений, одно из множеств $X X$ и $X+X$ имеет мощность намного большую, чем $|X|$, то можно ожидать, что в перспективе удалось бы заменить трилинейную сумму на некоторую билинейную, в котором одно из множеств будет иметь мощность значительно большую, чем мощность множества $X$. Такая замена дала бы возможность воспользоваться оценкой билинейной суммы (1). При попытке реализовать эту идею возникают проблемы, касающиеся мощностей так называемых условных сумм множеств. Эти проблемы решаются с помощью оценок типа Балога-Семереди-Гауэрса. Этими вопросами мы займемся в последующих разделах.

ОБознАчЕНИя. Всюду в статье $\log x$ означает максимум между 1 и натуральным логарифмом от $x$. Будем пользоваться сокращением

$$
e_{m}(z):=e^{2 \pi i z / m}
$$

Если $X$ является подмножеством некоторой группы по сложению и $k$ - натуральное число, то будем полагать $k X:=\left\{x_{1}+\cdots+x_{k}: x_{1}, \ldots, x_{k} \in X\right\}$. Если $X \subset \mathbb{F}_{p}, z \in \mathbb{F}_{p}$, то воспользуемся обозначением

$$
z X:=\{z x: x \in X\}
$$

Эти обозначения будут понятны по контексту и к недоразумениям привести не должны. Также временами можем для краткости писать $X^{2}$ вместо $X \times X$, $X^{3}$ вместо $X \times X \times X$ и т. д. 


\section{2. Оценки сумм множеств}

При доказательстве оценки сумм-произведений используется ряд утверждений из аддитивной комбинаторики по оценкам сумм множеств. В настоящем разделе мы даем обзор некоторых результатов по этому направлению. Мы ограничимся рассмотрением только подмножеств абелевых групп (по сложению), хотя некоторые из утверждений имеют место и для произвольных групп.

2.1. Неравенство треугольника Ружи. Одним из самых простых и полезных инструментов в исследовании ряда задач аддитивной комбинаторики является следующее утверждение, принадлежащее Ружи [32].

Лемма 2.1. Пусть $X, Y, Z$ - непустые конечные подмножества некоторой абелевой группы. Тогда

$$
|X-Z| \leqslant \frac{|X-Y||Y-Z|}{|Y|} .
$$

Неравенство в лемме 2.1 называется неравенством треугольника Ружи. Оно доказывается следующим образом. Каждому фиксированному элементу $t \in$ $X-Z$ сопоставляется фиксированное представление вида

$$
t=x(t)-z(t), \quad x(t) \in X, \quad z(t) \in Z .
$$

Затем рассматривается отображение

$$
f:(X-Z) \times Y \rightarrow(X-Y) \times(Y-Z),
$$

определяемое следующим образом: если $(t, y) \in(X-Z) \times Y$, то полагаем

$$
f((t, y))=(x(t)-y, y-z(t)) .
$$

Построенное отображение является инъекцией. Действительно, по заданной паре $(x(t)-y, y-z(t))$ однозначно определяется элемент

$$
t=(x(t)-y)+(y-z(t)) .
$$

Последнее, в свою очередь, однозначно определяет элементы $x(t)$ и $z(t)$. Следовательно, элемент $y$ также определяется однозначно.

Таким образом, мощность множества $(X-Z) \times Y$ не превосходит мощности множества $(X-Y) \times(Y-Z)$, откуда следует искомое неравенство

$$
|X-Z||Y| \leqslant|X-Y||Y-Z| \text {. }
$$

Отметим, что имеется еще одно неравенство треугольника Ружи,

$$
|X+Z| \leqslant \frac{|X+Y||Y+Z|}{|Y|},
$$

которое, однако, не так просто доказывается. Это неравенство было высказано в виде предположения в вышеупомянутой работе Ружи [32] и было доказано им 
в работе [33]. Оно выводится из неравенства Ружи-Плюнеке, которое, в свою очередь, основано на свойствах графов Плюнеке.

Одним из следствий неравенств треугольника Ружи является то, что если для множества $A$ выполняется оценка $|A-A|<|A|^{1+\varepsilon}$, то $|A+A|<|A|^{1+2 \varepsilon}$. Другими словами, множества, имеющие маленькие разности, также имеют маленькие удвоения. Обратное тоже справедливо: множества, имеющие маленькие удвоения, также имеют маленькие разности. Из неравенств треугольника Ружи также следует, что если $|A|=|B|=N$, причем $|A+B|<N^{1+\varepsilon}$, то каждое из множеств $A+A, A-A, B+B, B-B$ имеет мощность, не превосходящую $N^{1+2 \varepsilon}$.

2.2. Графы Плюнеке и их свойства. В последующих разделах мы будем многократно пользоваться неравенством Ружи-Плюнеке сумм множеств. Как уже было отмечено, вывод этого неравенства основан на графах Плюнеке и их свойствах.

Графом уровня $n$ называется граф $G=(V, E)$, в котором множество вершин $V$ является объединением непересекающихся множеств $V_{0}, V_{1}, \ldots, V_{n}$ таких, что каждое ребро $e \in E$ состоит из упорядоченной пары $\left(v, v^{\prime}\right)$, где $v \in V_{i-1}, v^{\prime} \in V_{i}$ для некоторого $1 \leqslant i \leqslant n$. Последовательность ребер вида $\left(v_{i-1}, v_{i}\right),\left(v_{i}, v_{i+1}\right), \ldots,\left(v_{j-1}, v_{j}\right)$ называется путем, соединяющим вершину $v_{i-1} \in V_{i-1}$ с вершиной $v_{j} \in V_{j}$. Для данного графа $G$ уровня $n$ определяется $i$-й коэффициент растяжения

$$
D_{i}=D_{i}(G)=\min _{X \subset V_{0}, X \neq \varnothing} \frac{\left|\operatorname{im}_{i}(X)\right|}{|X|},
$$

где $\operatorname{im}_{i}(X)$ - это множество всех вершин $v_{i} \in V_{i}$ таких, что существует путь, соединяющий некоторую вершину $v_{0} \in X$ с вершиной $v_{i}$.

Если $G=(V(G), E(G))$ и $H=(V(H), G(H))$ являются графами уровня $n$ с соответствующими множествами вершин

$$
V(G)=\bigcup_{i=0}^{n} V_{i}, \quad V(H)=\bigcup_{i=0}^{n} W_{i},
$$

то их произведение $G \times H$ определим следующим образом: за множество вершин графа $G \times H$ берем множество

$$
V(G \times H)=\left(V_{0} \times W_{0}\right) \cup\left(V_{1} \times W_{1}\right) \cup \cdots \cup\left(V_{n} \times W_{n}\right),
$$

а за множество ребер берем

$$
E(G \times H)=\left\{\left(\left(v_{i-1}, w_{i-1}\right),\left(v_{i}, w_{i}\right)\right):\left(v_{i-1}, v_{i}\right) \in E(G),\left(w_{i-1}, w_{i}\right) \in E(H)\right\} .
$$

В частности, произведение двух графов уровня $n$ также будет графом уровня $n$.

Простым и полезным свойством коэффициента растяжения является его мультипликативность. Именно, для любых двух графов $G$ и $H$ уровня $n$ имеет место равенство

$$
D_{i}(G \times H)=D_{i}(G) D_{i}(H) .
$$

Графом Плюнеке уровня $n$ называется граф $G=(V, E)$ уровня $n$ со следующими дополнительными свойствами. 
- Если $u, v, w_{1}, \ldots, w_{k} \in V$ таковы, что $(u, v) \in E$ и $\left(v, w_{j}\right) \in E$ для всех $1 \leqslant j \leqslant k$, то существуют $k$ различных вершин $v_{1}, \ldots, v_{k}$ таких, что $\left(u, v_{j}\right) \in E$ и $\left(v_{j}, w_{j}\right) \in E$ для всех $1 \leqslant j \leqslant k$.

- Если $u_{1}, \ldots u_{k}, v, w \in V$ таковы, что $\left(u_{j}, v\right) \in E$ для всех $1 \leqslant j \leqslant k$ и $(v, w) \in E$, то существуют $k$ различных вершин $v_{1}, \ldots, v_{k}$ таких, что $\left(u_{j}, v_{j}\right) \in E$ и $\left(v_{j}, w\right) \in E$ для всех $1 \leqslant j \leqslant k$.

Заметим, что произведение двух графов Плюнеке также является графом Плюнеке. Основным результатом теории графов Плюнеке является неравенство Плюнеке.

Теорема 2.1. Пусть $G$ - граф Плюнеке уровня $n$. Тогда выполняются неравенства

$$
D_{1}(G) \geqslant D_{2}(G)^{1 / 2} \geqslant \cdots \geqslant D_{n}(G)^{1 / n} .
$$

Важнейшим примером графа Плюнеке $G=(V, E)$ служит следующая конструкция. Пусть $A, B$ являются непустыми конечными подмножествами некоторой абелевой группы (по сложению). Положим $V=V_{0} \cup V_{1} \cup \cdots \cup V_{n}$, где $V_{0}=A \times\{0\}$ и

$$
V_{i}:=(A+i B) \times\{i\}=\left\{a+b_{1}+\cdots+b_{i}: a \in A, b_{j} \in B\right\} \times\{i\} .
$$

Дополнительные координаты $\{i\}$ вводятся для того, чтобы при $j \neq k$ множества $V_{j}$ и $V_{k}$ были непересекающимися. Множество ребер $E$ строится по принципу, что две вершины

$$
v_{j}=x_{j} \times\{j\} \in V_{j}, \quad v_{j+1}=x_{j+1} \times\{(j+1)\} \in V_{j+1}
$$

составляют ребро $\left(v_{j}, v_{j+1}\right) \in E$ тогда и только тогда, когда $x_{j+1}-x_{j} \in B$. Легко проверить, что построенный таким образом граф является графом Плюнеке.

Теперь заметим, что если $X \subset A$, то в приведенном примере будем иметь

$$
\operatorname{im}_{i}(X \times\{0\})=(X+i B) \times\{i\}=\left\{x+b_{1}+\cdots+b_{i}: x \in X, b_{j} \in B\right\} \times\{i\} .
$$

Таким образом, по определению коэффициента растяжения,

$$
D_{i}=\min _{X \subset A, X \neq \varnothing} \frac{|X+i B|}{|X|} .
$$

В частности,

$$
D_{i} \leqslant \frac{|A+i B|}{|A|} .
$$

Следовательно, согласно теореме 2.1 , при $k \geqslant i$ будем иметь

$$
\left(\frac{|A+i B|}{|A|}\right)^{1 / i} \geqslant\left(\inf _{X \subset A, X \neq \varnothing} \frac{|X+k B|}{|X|}\right)^{1 / k} .
$$

Предполагая, что при данном $k$ минимум в правой части достигается при $X=$ $X_{1} \subset A$, получим следующее утверждение. 
СледствиЕ 2.1. Пусть $A$ и $B$ - непустые конечные подмножества некоторой абелевой группь, $i$ и $k$ - фиксированные натуральные числа, $i \leqslant k$. Тогда существует непустое подмножество $X_{1} \subset A$ такое, что

$$
\left|X_{1}+k B\right| \leqslant\left(\frac{|A+i B|}{|A|}\right)^{k / i}\left|X_{1}\right| \text {. }
$$

В частности, так как $1 \leqslant\left|X_{1}\right| \leqslant|A|$, то, взяв $i=1$, получим

$$
|k B| \leqslant \frac{|A+B|^{k}}{|A|^{k-1}} .
$$

Доказательство самой теоремы 2.1 основано на следующей лемме.

Лемма 2.2. Пусть $G=(V, E)$ - граф Плюнеке уровня $n, V=\bigcup_{i=0}^{n} V_{i}$. Если $D_{n} \geqslant 1$, то существуют $\left|V_{0}\right|$ попарно непересекающихся путей из $V_{0}$ в $V_{n}$.

Из леммы 2.2 легко вывести, что если $D_{n} \geqslant 1$, то $D_{i} \geqslant 1$ при всех $1 \leqslant i \leqslant n$. При выводе теоремы 2.1 из леммы 2.2 помимо самого графа $G$ также используется следующий граф Плюнеке уровня $n$, который зависит от параметра $m$. Пусть $V_{0}=\{0\}$, и пусть $V_{1}$ состоит из $m$ натуральных чисел таких, что всевозможные суммы вида

$$
x_{1}+\cdots+x_{n}, \quad 0 \leqslant x_{1} \leqslant \cdots \leqslant x_{n}, \quad x_{i} \in V_{1} \cup\{0\},
$$

являются попарно различными. При $1 \leqslant i \leqslant n$ полагаем

$$
V_{i}=i V_{1}=\left\{v_{11}+\cdots+v_{1 i}: v_{1 j} \in V_{1}\right\}
$$

Нетрудно видеть, что

$$
\left|V_{i}\right|=\frac{m(m+1) \cdots(m+i-1)}{i !} .
$$

Две вершины $v_{i} \in V_{i}$ и $v_{i+1} \in V_{i+1}$ составляют ребро $\left(v_{i}, v_{i+1}\right) \in E$ тогда и только тогда, когда $v_{i+1}-v_{i} \in V_{1}$. Обозначим этот граф через $I_{m, n}$. Ясно, что

$$
\left|\operatorname{im}_{i}\left(V_{0}\right)\right|=\left|V_{i}\right|
$$

Поэтому

$$
m^{i} \geqslant \frac{m(m+1) \cdots(m+i-1)}{i !}=D_{i}\left(I_{m, n}\right) \geqslant \frac{m^{i}}{i !} .
$$

Для того, чтобы из леммы 2.2 вывести теорему 2.1, достаточно показать, что при $i<n$ выполняется неравенство $D_{i}(G) \geqslant D_{n}(G)^{i / n}$. Пусть сначала $0<D_{n}<1$. Тогда при любых натуральных числах $r$ и $m$ имеем

$$
D_{n}\left(G^{r} \times I_{m, n}\right)=D_{n}(G)^{r} D_{n}\left(I_{m, n}\right) \geqslant D_{n}(G)^{r} \frac{m^{n}}{n !} .
$$

Если $m=\left[(n !)^{1 / n} D_{n}(G)^{-r / n}\right]+1$, то будем иметь $D_{n}\left(G^{r} \times I_{m, n}\right) \geqslant 1$. Поэтому из леммы 2.2 следует, что

$$
D_{i}(G)^{r} m^{i} \geqslant D_{i}(G)^{r} D_{i}\left(I_{m, n}\right)=D_{i}\left(G^{r} \times I_{m, n}\right) \geqslant 1 .
$$


Таким образом,

$$
D_{i}(G) \geqslant m^{-i / r} \geqslant\left((n !)^{1 / n} D_{n}(G)^{-r / n}+1\right)^{-i / r} .
$$

Устремляя $r$ к бесконечности, получим требуемое (при $0<D_{n}<1$ ).

Если $D_{n}=1$, то по лемме 2.2 имеем $D_{i} \geqslant 1$, откуда следует требуемый результат.

Случай $D_{n}>1$ аналогичен случаю $0<D_{n}<1$. Но при этом вместо графа $I_{m, n}$ следует применить обратный граф $I_{m, n}^{-1}$, который строим из графа $I_{m, n}$ с помощью замены множества вершин $V_{i}$ на множества $W_{i}=V_{n-i}$, полагая $\left(w_{i}, w_{i+1}\right)$ ребром нового графа тогда и только тогда, когда $\left(w_{i+1}, w_{i}\right)$ является ребром графа $I_{m, n}$. При этом нужно учесть, что

$$
D_{n}\left(I_{m, n}^{-1}\right)=\frac{1}{\left|n V_{1}\right|} \geqslant m^{-n}, \quad D_{i}\left(I_{m, n}^{-1}\right) \leqslant \frac{(n-i) V_{1}}{n V_{1}} \leqslant n ! m^{-i} .
$$

Подробное доказательство теоремы 2.1 можно найти в монографиях [34] и [28]. Отметим только, что доказательство самой леммы 2.2 использует теорему Менгера из теории графов.

2.3. Неравенство Ружи-Плюнеке. В приложении нам будет более удобен следующий вариант следствия 2.1, который был доказан в работах Ружи [33], [35].

Лемма 2.3. Пусть $X, B_{1}, \ldots, B_{k}$ - непустые конечные подмножества некоторой абелевой группы, и пусть

$$
|X|=n, \quad\left|X+B_{i}\right|=\alpha_{i} n \quad(i=1, \ldots, k) .
$$

Тогда существует непустое подмножество $X_{1} \subset X$ mакое, что

$$
\left|X_{1}+B_{1}+\cdots+B_{k}\right| \leqslant \alpha_{1} \cdots \alpha_{k}\left|X_{1}\right| \text {. }
$$

СЛЕДСТВИЕ 2.2. Пусть $X, B_{1}, \ldots, B_{k}$ - непустые конечные подмножества некоторой абелевой группь. Тогда

$$
\left|B_{1}+\cdots+B_{k}\right| \leqslant \frac{\left|X+B_{1}\right| \cdots\left|X+B_{k}\right|}{|X|^{k-1}} .
$$

Взяв $k=2$, получим вышеупомянутый вариант неравенства треугольника Ружи

$$
|X+Z| \leqslant \frac{|X+Y||Y+Z|}{|Z|} .
$$

Приведем доказательство слегка более слабой формы леммы 2.3, с заменой

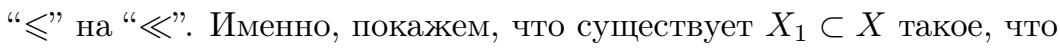

$$
\left|X_{1}+B_{1}+\cdots+B_{k}\right| \ll \frac{\left|X+B_{1}\right| \cdots\left|X+B_{k}\right|}{|X|^{k}}\left|X_{1}\right|,
$$

где константа в знаке « может зависеть от $k$. Такая формулировка на самом деле является достаточной для применений к получению нужных нам оценок сумм-произведений. 
Пусть $R$ - абелева группа, к которой принадлежат множества $X, B_{1}, \ldots, B_{k}$. Возьмем произвольное число $M>10 k\left(\left|X+B_{1}\right|+\cdots+\left|X+B_{k}\right|\right)$ и определим

$$
n_{j}=\left[\frac{M}{\left|X+B_{j}\right|}\right], \quad 1 \leqslant j \leqslant k .
$$

Будем работать в абелевой группе $R \times \mathbb{Z}$. Определим попарно различные целые числа $m_{i j}$, где $1 \leqslant i \leqslant k, 1 \leqslant j \leqslant n_{i}$, так, чтобы всевозможные суммы вида

$$
m_{1 j_{1}}+m_{2 j_{2}}+\cdots+m_{k j_{k}}
$$

были попарно различными. Определим множества

$$
B_{i j}=B_{i} \times\left\{m_{i j}\right\}, \quad 1 \leqslant i \leqslant k, \quad 1 \leqslant j \leqslant n_{i} .
$$

Эти множества являются попарно непересекающимися. Пусть

$$
Y=B_{11} \cup \cdots \cup B_{1 n_{1}} \cup B_{21} \cup \cdots \cup B_{2 n_{2}} \cup \cdots \cup B_{k 1} \cup \cdots \cup B_{k n_{k}} .
$$

Применим следствие 2.1 к множествам $X \times\{0\}$ и $Y$. Тогда получим, что существует подмножество $X_{1} \times\{0\} \subset X \times\{0\}$ такое, что

$$
\left|X_{1} \times\{0\}+k Y\right| \leqslant \frac{|X \times\{0\}+Y|^{k}}{|X|^{k}}\left|X_{1}\right| .
$$

Заметим, что $\left|X \times\{0\}+B_{i j}\right|=\left|X+B_{i}\right|$. Таким образом,

$$
|X \times\{0\}+Y| \leqslant n_{1}\left|X+B_{1}\right|+\cdots+n_{k}\left|X+B_{k}\right| \leqslant k M .
$$

По выбору чисел $m_{i j}$, разным векторам $\left(j_{1}, \ldots, j_{k}\right)$ соответствуют непересекающиеся множества

$$
X_{1} \times\{0\}+B_{1 j_{1}}+\cdots+B_{k j_{k}} .
$$

Количество таких множеств равно $n_{1} \cdots n_{k}$, а так как $M$ выбрано большим, то имеем

$$
n_{1} \cdots n_{k} \geqslant \frac{M^{k}}{2^{k}\left|X+B_{1}\right| \cdots\left|X+B_{k}\right|} .
$$

Мощность каждого из этих попарно непересекающихся множеств равна $\mid X_{1}+$ $B_{1}+\cdots+B_{k} \mid$. Так как все они содержатся в множестве $X_{1} \times\{0\}+k Y$, то получим

$$
\left|X_{1} \times\{0\}+k Y\right| \geqslant \frac{M^{k}}{2^{k}\left|X+B_{1}\right| \cdots\left|X+B_{k}\right|}\left|X_{1}+B_{1}+\cdots+B_{k}\right| .
$$

Требуемая оценка следует из (8)-(10).

2.4. Оценка типа Балога-Семереди-Гауэрса. Для подмножества $E \subset$ $A \times B$ введем обозначения

$$
A \underset{E}{+} B=\{a+b:(a, b) \in E\}, \quad A \underset{E}{A} B=\{a-b:(a, b) \in E\} .
$$


Суть оценок типа Балога-Семереди-Гауэрса заключается, грубо говоря, в том, что если множества $A$ и $B$ имеют соизмеримые по порядку мощности, если подмножество $E \subset A \times B$ - большое, а мощность множества $A+B$ мала, то существуют большие подмножества $A^{\prime} \subset A, B^{\prime} \subset B$ такие, что $A^{\prime}+B^{\prime}$ - маленькое. Результаты такого содержания впервые появились в работе Балога и Семереди [36]. В работе [37] Гауэрс внес существенно новую идею, которая позволила получить сильные оценки в ряде задач.

В литературе имеется ряд оценок типа Балога-Семереди-Гауэрса (см., например, работу Балога [38], работу Судакова, Семереди и Ву [39], монографию Тао и Ву [28]). Одним из удобных в применении к оценкам тригонометрических сумм является вариант, предложенный в работе [31].

Лемма 2.4. Пусть $А$ и $B$ являются конечными подмножествами некоторой абелевой группь (по сложению), и пусть $E \subset A \times B$ таково, что $|E| \geqslant|A||B| / K$. Тогда существует подмножество $A^{\prime} \subset A$ такое, ито $\left|A^{\prime}\right| \geqslant$ $0.1|A| / K u$

$$
|A+\underset{E}{+} B|^{4} \geqslant \frac{\left|A^{\prime}-A^{\prime}\right||A||B|^{2}}{10^{4} K^{5}}, \quad|A-B|^{4} \geqslant \frac{\left|A^{\prime}-A^{\prime}\right||A||B|^{2}}{10^{4} K^{5}} .
$$

Для данного $a \in A$ через $N(a)$ обозначим множество всех элементов $b \in B$, для которых $(a, b) \in E$. Соответственно, для данного элемента $b \in B$ через $N_{1}(b)$ обозначим множество всех элементов $a \in A$, для которых $(a, b) \in E$. В частности, если $b \in N(a)$, то $a+b \in \underset{E}{A+B}, a-b \in \underset{E}{A-B}$. Полезно заметить, что

(i) $a \in N_{1}(b) \Leftrightarrow b \in N(a)$;

(ii) $N\left(a_{1}\right) \cap N\left(a_{2}\right)=\left\{b \in B: a_{1} \in N_{1}(b), a_{2} \in N_{1}(b)\right\}$;

(iii) $\sum_{a \in A}|N(a)|=\sum_{b \in B}\left|N_{1}(b)\right|=|E|$.

Доказательство леммы 2.4 проходит по схеме работ [38], [39] и основано на следующей лемме (которая содержится в [28; лемма 6.19]).

Лемма 2.5. Пусть для некоторого $K \geqslant 1$ подмножество $E \subset A \times B$ имеет мощность $|E| \geqslant|A||B| / K$. Тогда для любого $0<\varepsilon<1$ существует подмножество $A^{\prime} \subset A$ мощности

$$
\left|A^{\prime}\right| \geqslant \frac{|A|}{\sqrt{2} K}
$$

такое, что по менъшей мере для $(1-\varepsilon)\left|A^{\prime}\right|^{2}$ nар $\left(a_{1}^{\prime}, a_{2}^{\prime}\right) \in A^{\prime} \times A^{\prime}$ имеет место оценка

$$
\left|N\left(a_{1}^{\prime}\right) \cap N\left(a_{2}^{\prime}\right)\right| \geqslant \frac{\varepsilon}{2 K^{2}}|B| .
$$

Для доказательства леммы 2.5 положим

$$
\Omega=\left\{\left(a_{1}, a_{2}\right) \in A \times A:\left|N\left(a_{1}\right) \cap N\left(a_{2}\right)\right|<\frac{\varepsilon}{2 K^{2}}|B|\right\} .
$$


Тогда по неравенству Коши-Буняковского

$$
\begin{aligned}
\sum_{b \in B}\left(\left|N_{1}(b)\right|^{2}-\frac{1}{\varepsilon} \sum_{\substack{\left(a_{1}, a_{2}\right) \in \Omega \\
a_{1}, a_{2} \in N_{1}(b)}} 1\right) \geqslant \frac{1}{|B|}\left(\sum_{b \in B}\left|N_{1}(b)\right|\right)^{2} \\
\quad-\frac{1}{\varepsilon} \sum_{\left(a_{1}, a_{2}\right) \in \Omega}\left|N\left(a_{1}\right) \cap N\left(a_{2}\right)\right| \geqslant \frac{|A|^{2}|B|}{K^{2}}-\frac{|\Omega||B|}{2 K^{2}} \geqslant \frac{|A|^{2}|B|}{2 K^{2}} .
\end{aligned}
$$

Таким образом, для некоторого $b=b_{0}$ имеем

$$
\left|N_{1}\left(b_{0}\right)\right|^{2}-\frac{1}{\varepsilon} \sum_{\substack{\left(a_{1}, a_{2}\right) \in \Omega \\ a_{1}, a_{2} \in N_{1}\left(b_{0}\right)}} 1 \geqslant \frac{|A|^{2}}{2 K^{2}} .
$$

Легко проверить, что множество $A^{\prime}=N_{1}\left(b_{0}\right)$ удовлетворяет утверждению леммы.

Теперь покажем, как из леммы 2.5 вывести лемму 2.4. Пару $\left(a_{1}, a_{2}\right) \in A \times A$ будем называть хорошей, если

$$
\left|N\left(a_{1}\right) \cap N\left(a_{2}\right)\right| \geqslant 0.05 \frac{|B|}{K^{2}} .
$$

В лемме 2.5 возьмем $\varepsilon=0.1$. Следовательно, для некоторого подмножества $A^{\prime} \subset A$ мощности $\left|A^{\prime}\right| \geqslant|A| / \sqrt{2} K$ имеется как минимум $0.9\left|A^{\prime}\right|^{2}$ хороших пар $\left(a_{1}^{\prime}, a_{2}^{\prime}\right) \in A^{\prime} \times A^{\prime}$. Для данного $a_{1}^{\prime} \in A^{\prime}$ через $I_{a_{1}^{\prime}}$ обозначим множество всех элементов $a_{2}^{\prime} \in A^{\prime}$, для которых $\left(a_{1}^{\prime}, a_{2}^{\prime}\right)$ является хорошей. Тогда

$$
\sum_{a^{\prime} \in A^{\prime}}\left|I_{a^{\prime}}\right| \geqslant 0.9\left|A^{\prime}\right|^{2}
$$

Пусть $A^{\prime \prime}$ является множеством всех элементов $a^{\prime \prime} \in A^{\prime}$, для которых $\left|I_{a^{\prime \prime}}\right| \geqslant$ $0.7\left|A^{\prime}\right|$. Имеем $\left|A^{\prime \prime}\right| \geqslant 0.2\left|A^{\prime}\right| \geqslant 0.1|A| / K$. Достаточно доказать, что

$$
|A+B|^{4} \geqslant \frac{\left|A^{\prime \prime}-A^{\prime \prime}\right||A||B|^{2}}{10^{4} K^{5}}, \quad|A-B|^{4} \geqslant \frac{\left|A^{\prime \prime}-A^{\prime \prime}\right||A||B|^{2}}{10^{4} K^{5}} .
$$

Так как для любых элементов $a_{1}^{\prime \prime}, a_{2}^{\prime \prime} \in A^{\prime \prime}$ множества $I_{a_{1}^{\prime \prime}}$ и $I_{a_{2}^{\prime \prime}}$ являются подмножествами множества $A^{\prime}$ мощности большей, чем $0.7\left|A^{\prime}\right|$, то по принципу Дирихле имеем

$$
\left|I_{a_{1}^{\prime \prime}} \cap I_{a_{2}^{\prime \prime}}\right| \geqslant 0.4\left|A^{\prime}\right| \geqslant 0.1 \frac{|A|}{K} .
$$

По определению, если $a \in I_{a_{1}^{\prime \prime}} \cap I_{a_{2}^{\prime \prime}}$, то обе пары $\left(a, a_{1}^{\prime \prime}\right)$ и $\left(a, a_{2}^{\prime \prime}\right)$ являются хорошими. Поэтому

$$
\left|N(a) \cap N\left(a_{1}^{\prime \prime}\right)\right| \geqslant 0.05 \frac{|B|}{K^{2}}, \quad\left|N(a) \cap N\left(a_{2}^{\prime \prime}\right)\right| \geqslant 0.05 \frac{|B|}{K^{2}} .
$$

Далее, для любых

$$
a \in I_{a_{1}^{\prime \prime}} \cap I_{a_{2}^{\prime \prime}}, \quad b_{1} \in N(a) \cap N\left(a_{1}^{\prime \prime}\right), \quad b_{2} \in N(a) \cap N\left(a_{2}^{\prime \prime}\right)
$$


запишем

$$
a_{1}^{\prime \prime}-a_{2}^{\prime \prime}=\left(a_{1}^{\prime \prime}+b_{1}\right)-\left(a+b_{1}\right)+\left(a+b_{2}\right)-\left(a_{2}^{\prime \prime}+b_{2}\right) .
$$

Следовательно, согласно (11) и (12), любой заданный элемент $a_{1}^{\prime \prime}-a_{2}^{\prime \prime} \in A^{\prime \prime}-A^{\prime \prime}$ имеет не меньше $|A||B|^{2} /\left(10^{4} K^{5}\right)$ различных представлений в виде $d_{1}-d_{2}+$ $d_{3}-d_{4}$, где $d_{i} \in A \underset{E}{+} B$ (в частности, разным элементам вида $a_{1}^{\prime \prime}-a_{2}^{\prime \prime}$ соответствуют попарно различные множества векторов $\left.\left(d_{1}, d_{2}, d_{3}, d_{4}\right)\right)$. Так как общее число векторов $\left(d_{1}, d_{2}, d_{3}, d_{4}\right)$ не превосходит величины $|A \underset{E}{+} B|^{4}$, то получим, что

$$
|A+\underset{E}{+} B|^{4} \geqslant \frac{\left|A^{\prime \prime}-A^{\prime \prime}\right||A||B|^{2}}{10^{4} K^{5}} .
$$

Заменяя же тождество (13) на

$$
a_{1}^{\prime \prime}-a_{2}^{\prime \prime}=\left(a_{1}^{\prime \prime}-b_{1}\right)-\left(a-b_{1}\right)+\left(a-b_{2}\right)-\left(a_{2}^{\prime \prime}-b_{2}\right),
$$

заключаем, что также выполняется оценка

$$
|A \underset{E}{-} B|^{4} \geqslant \frac{\left|A^{\prime \prime}-A^{\prime \prime}\right||A||B|^{2}}{10^{4} K^{5}} .
$$

\section{3. Оценки сумм-произведений}

Аддитивной энергией множеств $X$ и $Y$ называется величина $E_{+}(X, Y)$, определяемая равенством

$$
E_{+}(X, Y)=\#\left\{\left(x_{1}, x_{2}, y_{1}, y_{2}\right) \in X^{2} \times Y^{2}: x_{1}+y_{1}=x_{2}+y_{2}\right\}
$$

Аналогично, мультипликативной энергией множеств $X$ и $Y$ называется величина $E_{\times}(X, Y)$, определяемая равенством

$$
E_{\times}(X, Y)=\#\left\{\left(x_{1}, x_{2}, y_{1}, y_{2}\right) \in X^{2} \times Y^{2}: x_{1} y_{1}=x_{2} y_{2}\right\} .
$$

Из неравенства Коши-Буняковского легко вывести, что

$$
|X+Y| \geqslant \frac{|X|^{2}|Y|^{2}}{E_{+}(X, Y)}, \quad|X Y| \geqslant \frac{|X|^{2}|Y|^{2}}{E_{\times}(X, Y)} .
$$

Действительно, если $I(\lambda)$ обозначает число решений уравнения

$$
x+y=\lambda, \quad x \in X, \quad y \in Y,
$$

то

$$
E_{+}(X, Y)=\sum_{\lambda \in X+Y} I^{2}(\lambda) \geqslant \frac{1}{|X+Y|}\left(\sum_{\lambda \in X+Y} I(\lambda)\right)^{2}=\frac{|X|^{2}|Y|^{2}}{|X+Y|} .
$$

Аналогично выводится нижняя оценка величины $|X Y|$.

Очевидно, что $E_{+}(X, Y)=E_{+}(Y, X)=E_{+}(X,-Y)$. Поэтому верхняя оценка для $E_{+}(X, Y)$ влечет нижнюю оценку не только для величины $|X+Y|$, но также и для величины $|X-Y|$. 
Лемма 3.1. Пусть $X, Y \subset \mathbb{F}_{p}$. Тогда существует элемент $z \in \mathbb{F}_{p}$ такой, что

$$
|X+z Y| \geqslant \frac{1}{2} \min \{|X||Y|, p\}
$$

ДоКАЗАТЕЛЬСтво. Можно считать, что $|Y| \geqslant 2$. Пусть $I$ - число решений уравнения

$$
x_{1}+z y_{1}=x_{2}+z y_{2}, \quad x_{1}, x_{2} \in X, \quad y_{1}, y_{2} \in Y, \quad z \in \mathbb{F}_{p} .
$$

Если $y_{1}=y_{2}$, то $x_{1}=x_{2}$; в этом случае получаем не более, чем $p|X||Y|$, решений. Если $y_{1} \neq y_{2}$, то по каждому данному вектору $\left(x_{1}, x_{2}, y_{1}, y_{2}\right)$ элемент $z$ определяется однозначно. Поэтому

$$
I \leqslant p|X||Y|+|X|^{2}|Y|^{2} .
$$

Следовательно, для некоторого фиксированного $z \in \mathbb{F}_{p}$ число решений уравнения

$$
x_{1}+z y_{1}=x_{2}+z y_{2}, \quad x_{1}, x_{2} \in X, \quad y_{1}, y_{2} \in Y,
$$

не превосходит величины $|X||Y|+|X|^{2}|Y|^{2} p^{-1}$. Если $z=0$, то указанное уравнение имеет $|X||Y|^{2}$ решений, откуда, ввиду $|Y| \geqslant 2$, получим $|X| \geqslant p / 2$ и, следовательно, в этом случае лемма доказана.

Если же $z \neq 0$, то имеем

$$
E_{+}(X, z Y) \leqslant|X||Y|+\frac{|X|^{2}|Y|^{2}}{p} .
$$

Так как $E_{+}(X, z Y) \geqslant|X|^{2}|Y|^{2} /|X+z Y|$, утверждение леммы следует.

Следующее важное утверждение вытекает из работы Глибичука и Конягина [26].

Лемма 3.2. Пусть $A_{1} \subset \mathbb{F}_{p}$. Тогда существуют элементы $b_{1}, b_{2}, b_{3}, b_{4} \in A_{1}$ такие, что либо

$$
\left|\left(b_{1}-b_{2}\right) A_{1}+\left(b_{3}-b_{4}\right) A_{1}+\left(b_{3}-b_{4}\right) A_{1}\right| \gg\left|A_{1}\right|^{2},
$$

либо же

$$
\left|\left(b_{1}-b_{2}\right) A_{1}+\left(b_{3}-b_{4}\right) A_{1}\right| \gg p .
$$

ДокАЗАТЕЛЬСтво. Можно считать, что $\left|A_{1}\right|>1$. Если

$$
\frac{A_{1}-A_{1}}{A_{1}-A_{1}}+1 \not \subset \frac{A_{1}-A_{1}}{A_{1}-A_{1}},
$$

то для некоторых элементов $b_{1}, b_{2}, b_{3}, b_{4} \in A_{1}$ таких, что $b_{3} \neq b_{4}$, имеем

$$
\frac{b_{1}-b_{2}}{b_{3}-b_{4}}+1 \notin \frac{A_{1}-A_{1}}{A_{1}-A_{1}} \text {. }
$$

Следовательно, отображение $f: A_{1} \times A_{1} \rightarrow \mathbb{F}_{p}$, определяемое условием

$$
f(u, v)=\left(\frac{b_{1}-b_{2}}{b_{3}-b_{4}}+1\right) u+v, \quad u, v \in A_{1},
$$


является инъекцией. Поэтому

$$
\left|\left(\frac{b_{1}-b_{2}}{b_{3}-b_{4}}+1\right) A_{1}+A_{1}\right|=\left|A_{1}\right|^{2},
$$

откуда следует утверждение в этом случае.

Если же

то очевидно имеем

$$
\frac{A_{1}-A_{1}}{A_{1}-A_{1}}+1 \subset \frac{A_{1}-A_{1}}{A_{1}-A_{1}},
$$

$$
\frac{A_{1}-A_{1}}{A_{1}-A_{1}}=\mathbb{F}_{p}
$$

Поэтому, согласно лемме 3.1 , существует элемент $z \in\left(A_{1}-A_{1}\right) /\left(A_{1}-A_{1}\right)$ такой, что

$$
\left|A_{1}+z A_{1}\right| \geqslant \frac{1}{2} \min \left\{p,\left|A_{1}\right|^{2}\right\} .
$$

Следовательно, для некоторого $\left(b_{1}, b_{2}, b_{3}, b_{4}\right) \in A_{1}^{4}$ имеем

$$
\left|\left(b_{1}-b_{2}\right) A_{1}+\left(b_{3}-b_{4}\right) A_{1}\right| \geqslant \frac{1}{2} \min \left\{p,\left|A_{1}\right|^{2}\right\} .
$$

Поэтому либо

$$
\left|\left(b_{1}-b_{2}\right) A_{1}+\left(b_{3}-b_{4}\right) A_{1}\right| \geqslant \frac{1}{2} p
$$

либо же

$$
\left|\left(b_{1}-b_{2}\right) A_{1}+\left(b_{3}-b_{4}\right) A_{1}\right| \geqslant \frac{1}{2}\left|A_{1}\right|^{2} .
$$

Лемма 3.2 доказана.

3.1. Доказательство оценки сумм-произведений. Докажем теорему 1.1. Ввиду предложения 1.1 , теорему достаточно доказать для случая ${ }^{6}$ $|A|<p^{1 / 2}$. Можно считать, что $|A|^{2} \geqslant 10|A A| \log |A|$ и $0 \notin A$. Заметим, что

$$
E_{\times}(A, A)=\sum_{a \in A} \sum_{b \in A}|a A \cap b A| .
$$

Поэтому для некоторого фиксированного элемента $b=b_{0} \in A$ будем иметь

$$
\sum_{a \in A}\left|a A \cap b_{0} A\right| \geqslant \frac{E_{\times}(A, A)}{|A|} .
$$

Распределяя величины $\left|a A \cap b_{0} A\right|$ в двоичные интервалы вида $\left[2^{j-1}, 2^{j}\right)$, легко получим, что для некоторого числа $N \geqslant 1$ и для некоторого подмножества $A_{1} \subset A$ выполняются следующие условия:

$$
\begin{gathered}
N \leqslant\left|a A \cap b_{0} A\right|<2 N \quad \text { для любого } a \in A_{1} ; \\
N\left|A_{1}\right| \geqslant \frac{E_{\times}(A, A)}{5|A| \log |A|} .
\end{gathered}
$$

\footnotetext{
${ }^{6}$ Действительно, если теорема доказана для всех $A$ мощности $|A|<p^{1 / 2}$, то это влечет, что теорема будет справедлива также при $p^{1 / 2}<|A|<p^{1 / 2+\delta_{1}}$, где $\delta_{1}$ - некоторая маленькая положительная константа. Если $p^{1-\varepsilon}>|A|>p^{1 / 2+\delta_{1}}$, то утверждение теоремы следует из предложения 1.1.
} 
В частности, так как $N \leqslant|A|$ и $\left|A_{1}\right| \leqslant|A|$, имеем

$$
N \geqslant \frac{E_{\times}(A, A)}{5|A|^{2} \log |A|}, \quad\left|A_{1}\right| \geqslant \frac{E_{\times}(A, A)}{5|A|^{2} \log |A|} .
$$

Так как $E_{\times}(A, A) \geqslant|A|^{4}|A A|^{-1}$, то $\left|A_{1}\right|>1$. Из неравенств треугольника Ружи и неравенства (14) выводим, что для любого элемента $a \in A_{1}$ и для любого выбора знака "士” имеет место оценка

$$
\left|a A \pm b_{0} A\right| \leqslant \frac{\left|a A+\left(a A \cap b_{0} A\right)\right|\left|\left(a A \cap b_{0} A\right)+b_{0} A\right|}{\left|a A \cap b_{0} A\right|} \leqslant \frac{|A+A|^{2}}{N} .
$$

Так как $A_{1} \subset A$, то, согласно лемме 3.2 , существуют элементы $b_{1}, b_{2}, b_{3}, b_{4} \in A_{1}$ такие, что

$$
\left|\left(b_{1}-b_{2}\right) A+\left(b_{3}-b_{4}\right) A+\left(b_{3}-b_{4}\right) A\right| \gg\left|A_{1}\right|^{2} .
$$

При этом можно считать, что $b_{1} \neq b_{2}$. Применим следствие 2.2 с $k=3$ и

$$
X=B_{1}=B_{2}=\left(b_{3}-b_{4}\right) A, \quad B_{3}=\left(b_{1}-b_{2}\right) A .
$$

Получим

$$
\left|A_{1}\right|^{2} \ll \frac{|A+A|^{2}\left|\left(b_{1}-b_{2}\right) A+\left(b_{3}-b_{4}\right) A\right|}{|A|^{2}} .
$$

Применим следствие 2.2 с $k=4$ и

$$
X=b_{0} A, \quad B_{1}=b_{3} A, \quad B_{2}=-b_{4} A, \quad B_{3}=b_{1} A, \quad B_{4}=-b_{2} A .
$$

Получим

$$
\left|\left(b_{1}-b_{2}\right) A+\left(b_{3}-b_{4}\right) A\right| \leqslant \frac{\left|b_{0} A+b_{3} A\right|\left|b_{0} A-b_{4} A\right|\left|b_{0} A+b_{1} A\right|\left|b_{0} A-b_{2} A\right|}{|A|^{3}} .
$$

Применяя к правой части (18) неравенство (16) и учитывая (17), получим

$$
|A+A|^{10} \gg\left|A_{1}\right|^{2}|A|^{5} N^{4} .
$$

Учитывая (14), а затем (15), получим, что

$$
|A+A|^{10} \gg \frac{E_{\times}(A, A)^{4}}{|A|}(\log |A|)^{-4} .
$$

Так как $E_{\times}(A, A) \geqslant|A|^{4}|A A|^{-1}$, то заключаем, что

$$
|A+A|^{10}|A A|^{4} \gg|A|^{15}(\log |A|)^{-4} .
$$

3.2. Вариант проблемы сумм-произведений с разными множествами. При выводе оценки сумм-произведений мы фактически доказали неравенство

$$
|A+A|+\frac{|A|^{4}}{E_{\times}(A, A)} \gg|A|^{1+\delta},
$$


которое, ввиду оценки $E_{\times}(A, A) \geqslant|A|^{4} /|A A|$, является более сильным утверждением, чем оценка сумм-произведений в оригинальной форме. Формулировка оценки сумм-произведений с привлечением мультипликативной энергии может быть удобна и в некоторых применениях, так как нетривиальная оценка мультипликативной энергии содержит в себе мультипликативную форму оценки типа Балога-Семереди-Гауэрса. Действительно, пусть имеет место нетривиальная оценка

$$
\frac{|A|^{4}}{E_{\times}(A, A)} \geqslant|A|^{1+\delta}, \quad \delta>0,
$$

и пусть $E$ - подмножество $A \times A$ мощности $|E| \geqslant \alpha|A|^{2}$, где $0<\alpha<1$. Так как уравнение

$$
a_{1} a_{2}=a_{3} a_{4}, \quad\left(a_{1}, a_{2}\right) \in E, \quad\left(a_{3}, a_{4}\right) \in E,
$$

имеет не более $E_{\times}(A, A)$ решений, то из неравенства Коши-Буняковского выводим, что

$$
|A \underset{E}{\times} A|=\left|\left\{a_{1} a_{2}:\left(a_{1}, a_{2}\right) \in E\right\}\right| \geqslant \frac{|E|^{2}}{E_{\times}(A, A)} \geqslant \alpha^{2}|A|^{1+\delta} .
$$

Тем самым имеем нижнюю оценку для мощности множеств $A \underset{E}{\times} A$.

Из вышесказанного следует, что было бы полезно иметь оценки сумм-произведений с привлечением мультипликативной энергии вместо произведения множеств. Такие оценки являются еще более значимыми, когда речь идет о мультипликативной энергии множеств, мощности которых между собой по порядку несоизмеримы.

Далее, из неравенств треугольника Ружи в аддитивной и мультипликативной формах следует, что если $A, B, C$ являются подмножествами $\mathbb{F}_{p}^{*}$, то

$$
|A+B|^{2} \geqslant|B||A+A|, \quad|A C|^{2} \geqslant|C||A A| .
$$

Следовательно, если

$$
|A|=|B|=|C|=M<p^{1-\varepsilon},
$$

то оценка сумм-произведений влечет, что

$$
\max \{|A+B|,|A C|\} \gg M^{1+\delta}, \quad \delta=\delta(\varepsilon)>0 .
$$

Учитывая это наблюдение, рассмотрим задачу получения нетривиальной оценки полилинейной тригонометрической суммы

$$
\sum_{x_{1} \in X_{1}} \cdots \sum_{x_{2 k-2} \in X_{2 k-2}} \sum_{x_{2 k-1} \in X_{2 k-1}} \sum_{x_{2 k} \in X_{2 k}} e_{p}\left(x_{1} \cdots x_{2 k-2} x_{2 k-1} x_{2 k}\right),
$$

где все множества $X_{i}$ имеют приблизительно одну и ту же мощность $\left|X_{i}\right| \sim M$. Мы хотим с помощью оценки сумм-произведений, оценок типа Балога-Семереди-Гауэрса и неравенства Коши-Буняковского заменить суммирование по двум переменным, скажем, по переменным $x_{2 k-1} \in X_{2 k-1}, x_{2 k} \in X_{2 k}$, на суммирование по одной переменной $y_{k}$, но по такому множеству $Y_{k}$, которое имеет мощность $\left|Y_{k}\right|>M^{1+\delta}$ с некоторой подходящей константой $\delta>0$. Тогда задача 
сводилась бы к оценке суммы

$$
\sum_{x_{1} \in X_{1}} \cdots \sum_{x_{2 k-2} \in X_{2 k-2}} \sum_{y_{k} \in Y_{k}} e_{p}\left(x_{1} \cdots x_{2 k-2} y_{k}\right)
$$

То же самое мы проделали бы с полученной суммой, заменяя переменные суммирования $x_{2 k-3}, x_{2 k-2}$ на суммирование по одной переменной $y_{k-1} \in Y_{k-1}$, при этом имея $\left|Y_{k-1}\right|>M^{1+\delta}$. Через $k$ шагов дошли бы до суммы вида

$$
\sum_{y_{1} \in Y_{1}} \cdots \sum_{y_{k} \in Y_{k}} e_{p}\left(y_{1} \cdots y_{k}\right)
$$

где все множества $Y_{i}$ уже имеют мощность $\left|Y_{i}\right|>M^{1+\delta}$. Теперь предполагая, что $k$ является степенью двойки, мы могли бы итерировать этот процесс нужное количество раз и в конечном итоге получили бы билинейную сумму по множествам большой мощности, к которой применима оценка (1).

Данному процессу можно придать строгий вид по следующей схеме. Пусть $X, Y$ - подмножества $\mathbb{F}_{p}^{*}$ такие, что $|X| \approx|Y| \approx M<p^{1 / 2}, N$ - натуральное число такое, что $N>p^{\varepsilon}$ для некоторой маленькой константы $\varepsilon>0$, и пусть $s_{1}, \ldots, s_{N}-$ произвольная последовательность ненулевых элементов поля $\mathbb{F}_{p}^{*}$. Пусть сумма

$$
\sum_{x \in X}\left|\sum_{y \in Y} \sum_{n=1}^{N} e_{p}\left(x y s_{n}\right)\right|
$$

не допускает нетривиальной оценки. Таким образом, предполагаем, что

$$
\sum_{x \in X}\left|\sum_{y \in Y} \sum_{n=1}^{N} e_{p}\left(x y s_{n}\right)\right|>M^{2+o(1)} N .
$$

Покажем, что тогда существуют подмножество $Z \subset \mathbb{F}_{p}^{*}$ и константа $\delta>0$ такие, что $|Z|>M^{1+\delta}$ и

$$
\sum_{z \in Z}\left|\sum_{n=1}^{N} e_{p}\left(z s_{n}\right)\right|>|Z|^{1+o(1)} N .
$$

Предположим противное, именно, если (20) имеет место, то $|Z| \leqslant M^{1+o(1)}$. Из (19) следует, что существует подмножество $X^{\prime} \subset X$ мощности $\left|X^{\prime}\right|=M^{1+o(1)}$ такое, что

$$
\left|\sum_{y \in Y} \sum_{n=1}^{N} e_{p}\left(x y s_{n}\right)\right|>M^{1+o(1)} N \quad \text { для любого } x \in X^{\prime} .
$$

(i) Из (21) легко вывести, что существует подмножество $E \subset X^{\prime} \times Y$ мощности $|E|=M^{2+o(1)}$ такое, что для любого $(x, y) \in E$ имеет место оценка

$$
\left|\sum_{n=1}^{N} e_{p}\left(x y s_{n}\right)\right|>N M^{o(1)}
$$

По предположению “от противного” имеем $\left|X^{\prime} \underset{E}{\times} Y\right|=M^{1+o(1)}$. Поэтому из оценки Балога-Семереди-Гауэрса в мультипликативной форме следует, 
что существует подмножество $X^{\prime \prime} \subset X^{\prime}$ мощности $\left|X^{\prime \prime}\right|=M^{1+o(1)}$ такое, что $\left|X^{\prime \prime} X^{\prime \prime}\right|=M^{1+o(1)}$.

(ii) Просуммируем (21) по множеству $x \in X^{\prime \prime}$. Получим

$$
\sum_{y \in Y} \sum_{n=1}^{N}\left|\sum_{x \in X^{\prime \prime}} \alpha(x) e_{p}\left(x y s_{n}\right)\right|>M^{2+o(1)} N,
$$

где $\alpha(x)$ - некоторые комплексные числа, равные по модулю единице. Фиксируя нужным образом элемент $y=y_{0}$ и применяя неравенство Коши-Буняковского по переменной $n$, получим

$$
\sum_{x_{1}, x_{2} \in X^{\prime \prime}}\left|\sum_{n=1}^{N} e_{p}\left(\left(x_{1}-x_{2}\right) y_{0} s_{n}\right)\right|>M^{2+o(1)} N \text {. }
$$

Таким образом, существует подмножество $E \subset X^{\prime \prime} \times X^{\prime \prime}$ мощности $|E|=$ $M^{2+o(1)}$ такое, что для любого $\left(x_{1}, x_{2}\right) \in E$ имеет место оценка

$$
\left|\sum_{n=1}^{N} e_{p}\left(\left(x_{1}-x_{2}\right) y_{0} s_{n}\right)\right|>N M^{o(1)} .
$$

По предположению "от противного" имеем $\left|X^{\prime \prime} \underset{E}{-} X^{\prime \prime}\right|=M^{1+o(1)}$. Поэтому из оценки Балога-Семереди-Гауэрса в аддитивной форме следует, что существует подмножество $X^{\prime \prime \prime} \subset X^{\prime \prime}$ мощности $\left|X^{\prime \prime \prime}\right|=M^{1+o(1)}$ такое, что

$$
\left|X^{\prime \prime \prime}+X^{\prime \prime \prime}\right|=M^{1+o(1)} \text {. }
$$

Остается заметить, что заключения пунктов (i) и (ii) вместе противоречат оценке сумм-произведений для $\max \left\{\left|X^{\prime \prime \prime} X^{\prime \prime \prime}\right|,\left|X^{\prime \prime \prime}+X^{\prime \prime \prime}\right|\right\}$.

Из вышеприведенной схемы и оценки билинейной суммы (1) легко вывести различные нетривиальные результаты по оценкам полилинейных тригонометрических сумм, в том числе результат Бургейна, Глибичука и Конягина [5]. Но можно доказывать специальные варианты сумм-произведений с несоизмеримыми по мощности множествами, которые позволяют для полилинейных сумм, при большом количестве переменных суммирования, получить более точные результаты, в частности, результат Бургейна оценки тригонометрических сумм по подгруппам $H$ порядка $|H|>e^{c \log p / \log \log p}$. С этой целью будем доказывать следующую версию оценок сумм-произведений.

Teоpema 3.1. Пусть $A, B \subset \mathbb{F}_{p}^{*}, L=\min \left\{|B|, p|A|^{-1}\right\}$. Тогда

$$
|A-A|^{2} \frac{|A|^{2}|B|^{2}}{E_{\times}(A, B)} \gg|A|^{3} L^{1 / 9}(\log L)^{-1} .
$$

Теорема 3.1 является явной версией оценки сумм-произведений Бургейна для разных множеств. Еще одна версия этой оценки, без привлечения мультипликативной энергии, имеется в работе [40]. Приложения теоремы 3.1 к оценкам тригонометрических сумм будут даны в разделе 4. В приложениях множество $B$ будет иметь сравнительно маленькую мощность. Если $L \rightarrow \infty$ при 
$p \rightarrow \infty$ и если $|B|<|A|$, то теорема 3.1 уже влечет нетривиальную оценку. Заметим, что мы сформулировали результат с разностью множеств $A-A$ для того, чтобы в приложении к оценкам тригонометрической суммы было более удобным применение оценки типа Балога-Семереди-Гауэрса (см. лемму 2.4, где присутствует множество $\left.A^{\prime}-A^{\prime}\right)$.

3.2.1. Леммы. Для доказательства теоремы 3.1 нам понадобятся несколько дополнительных лемм.

Лемма 3.3. Пусть $X, Y-$ подмножества $\mathbb{F}_{p}^{*},|X| \geqslant 2,|Y| \geqslant 2$. Пусть для некоторого элемента $z_{0} \in \mathbb{F}_{p}^{*}$ и некоторого положительного числа $K$ выполняется условие

$$
\left|y X+z_{0} X\right| \leqslant K|X| \quad \text { для всех } y \in Y .
$$

Тогда существуют элементы $x_{1}, x_{2} \in X, y_{1}, y_{2} \in Y$ такие, что либо

$$
\left|\left(x_{1}-x_{2}\right) Y+\left(y_{1}-y_{2}\right) X+\left(y_{1}-y_{2}\right) X\right| \geqslant \frac{|X||Y|}{4 K},
$$

либо же

$$
\left|\left(x_{1}-x_{2}\right) Y+\left(y_{1}-y_{2}\right) X\right| \geqslant \frac{p}{2} .
$$

ДокАЗАТЕЛЬСтво. Можно предположить, что $|X|>2 K,|Y|>2 K$, так как в противном случае утверждение очевидно. Из условия $\left|y X+z_{0} X\right| \leqslant K|X|$ следует, что для любого $y \in Y$ имеет место оценка

$$
\#\left\{\left(x, x_{1}, x_{2}, x_{3}\right) \in X^{4}: z_{0} x+y x_{2}=z_{0} x_{1}+y x_{3}\right\} \geqslant \frac{|X|^{3}}{K} .
$$

Следовательно, число решений уравнения

$$
z_{0} x+y x_{2}=z_{0} x_{1}+y x_{3}, \quad\left(x, x_{1}, x_{2}, x_{3}, y\right) \in X^{4} \times Y,
$$

не меньше чем $|X|^{3}|Y| / K$. Если $x_{2}=x_{3}$, то $x=x_{1}$; в этом случае мы получим не более, чем $|X|^{2}|Y| \leqslant|X|^{3}|Y| /(2 K)$, решений. Поэтому для некоторого фиксированного $\left(x_{1}, x_{2}, x_{3}\right)=\left(x_{0}, x_{0}^{\prime}, x_{0}^{\prime \prime}\right) \in X^{3}$ с условием $x_{0}^{\prime} \neq x_{0}^{\prime \prime}$ будем иметь

$$
\left|z_{0}\left(X-x_{0}\right) \cap\left(x_{0}^{\prime \prime}-x_{0}^{\prime}\right) Y\right| \geqslant \frac{|Y|}{2 K} .
$$

Пусть

$$
Y_{1}=\frac{z_{0}}{x_{0}^{\prime \prime}-x_{0}^{\prime}}\left(X-x_{0}\right) \cap Y
$$

Тогда

$$
Y_{1} \subset Y, \quad \frac{x_{0}^{\prime \prime}-x_{0}^{\prime}}{z_{0}} Y_{1} \subset X-x_{0}, \quad\left|Y_{1}\right| \geqslant \frac{|Y|}{2 K}>1 .
$$

Применим идею Глибичука и Конягина [26]. Если

$$
\frac{X-X}{Y_{1}-Y_{1}} \neq \mathbb{F}_{p},
$$

то для некоторого $\left(x_{1}, x_{2}, y_{1}, y_{2}\right) \in X^{2} \times Y_{1}^{2}$ имеем

$$
\frac{x_{1}-x_{2}}{y_{1}-y_{2}}+\frac{x_{0}^{\prime \prime}-x_{0}^{\prime}}{z_{0}} \notin \frac{X-X}{Y_{1}-Y_{1}} .
$$


Следовательно,

$$
\left|\left(\frac{x_{1}-x_{2}}{y_{1}-y_{2}}+\frac{x_{0}^{\prime \prime}-x_{0}^{\prime}}{z_{0}}\right) Y_{1}+X\right|=|X|\left|Y_{1}\right| \geqslant \frac{|X||Y|}{2 K} .
$$

Так как

$$
\frac{x_{0}^{\prime \prime}-x_{0}^{\prime}}{z_{0}} Y_{1} \subset X-x_{0},
$$

то в рассматриваемом случае получаем требуемый результат.

Если

$$
\frac{X-X}{Y_{1}-Y_{1}}=\mathbb{F}_{p}
$$

то из леммы 3.1 следует, что существует $\left(x_{1}, x_{2}, y_{1}, y_{2}\right) \in X^{2} \times Y_{1}^{2}$ такое, что

$$
\left|\left(x_{1}-x_{2}\right) Y_{1}+\left(y_{1}-y_{2}\right) X\right| \geqslant \frac{1}{2} \min \left\{|X|\left|Y_{1}\right|, p\right\} \geqslant \frac{1}{2} \min \left\{\frac{|X||Y|}{2 K}, p\right\} .
$$

Это завершает доказательство леммы 3.3.

Следующая лемма позволяет избежать некоторых неудобств, которые появляются при доказательстве теоремы 3.1 в случае, когда величина $p /|A|$ меньше некоторой фиксированной степени $\log |B|$.

Лемма 3.4. Пусть $A, B, C \subset \mathbb{F}_{p}^{*}$. Тогда

$$
|A+C| \frac{|A|^{2}|B|^{2}}{E_{\times}(A, B)} \gg \min \left\{p|A|, \frac{|A|^{2}|B||C|}{p}\right\} .
$$

В частности, если $|A|(\log |B|)^{1000} \geqslant p$, то

$$
|A-A| \frac{|A|^{2}|B|^{2}}{E_{\times}(A, B)} \gg p|A| .
$$

ДокАЗАТЕЛЬСтво. Пусть $J$ - число решений уравнения

$$
a b b_{1}^{-1}+c=x, \quad\left(a, b, b_{1}, c, x\right) \in A \times B \times B \times C \times(A+C) .
$$

Для любого вектора $\left(a, a_{1}, b, b_{1}, c\right) \in A^{2} \times B^{2} \times C$ с условием $a b=a_{1} b_{1}$ вектор

$$
\left(a, b, b_{1}, c, a_{1}+c\right) \in A \times B \times B \times C \times(A+C)
$$

является решением уравнения (22). Разным векторам $\left(a, a_{1}, b, b_{1}, c\right) \in A^{2} \times$ $B^{2} \times C$ с условием $a b=a_{1} b_{1}$ соответствуют разные векторы $\left(a, b, b_{1}, c, a_{1}+c\right)$. Следовательно, число $J$ удовлетворяет неравенству

$$
J \geqslant|C| E_{\times}(A, B) .
$$

Выражая $J$ в терминах тригонометрических сумм и следуя методу доказательства предложения 1.1, получим

$$
J \leqslant \frac{|A||B|^{2}|C||A+C|}{p}+\sqrt{|C||A+C|} \max _{(n, p)=1} W(n),
$$


где

$$
W(n)=\left|\sum_{b_{1} \in B} \sum_{a \in A} \sum_{b \in B} e_{p}\left(n a b b_{1}^{-1}\right)\right| .
$$

Применяя неравенство Коши-Буняковского к сумме по $b_{1}$, при $(n, p)=1$ будем иметь

$$
W(n)^{2} \leqslant|B| \sum_{t=0}^{p-1}\left|\sum_{a \in A} \sum_{b \in B} e_{p}(n a b t)\right|^{2}=p|B| E_{\times}(A, B) .
$$

Таким образом,

$$
J \leqslant \frac{|A||B|^{2}|C||A+C|}{p}+\sqrt{|C||A+C|} \sqrt{p|B| E_{\times}(A, B)} .
$$

Сравнивая полученную оценку с неравенством (23), получим требуемое.

3.2.2. Доказательство теоремы 3.1. Можно предположить, что $|A|>10$, $|B|>10$. Если $|A|(\log |B|)^{200} \geqslant p$, то из леммы 3.4 следует, что

$$
|A-A|^{2} \frac{|A|^{2}|B|^{2}}{E_{\times}(A, B)} \gg p|A|^{2},
$$

откуда вытекает утверждение теоремы. Поэтому можно также считать, что выполняется условие $p /|A|>(\log |B|)^{200}$.

Пусть

$$
\frac{|A|^{2}|B|^{2}}{E_{\times}(A, B)}=|A| \Delta \text {. }
$$

Тогда

$$
\sum_{b \in B} \sum_{b^{\prime} \in B}\left|b A \cap b^{\prime} A\right|=E_{\times}(A, B)=\frac{|A||B|^{2}}{\Delta} .
$$

Следовательно, для некоторого фиксированного $b_{0} \in B$

$$
\sum_{b \in B}\left|b A \cap b_{0} A\right| \geqslant \frac{|A||B|}{\Delta} .
$$

Определим множество

$$
B_{1}=\left\{b \in B:\left|b A \cap b_{0} A\right| \geqslant \frac{|A|}{2 \Delta}\right\} .
$$

Имеем

$$
\sum_{b \in B_{1}}\left|b A \cap b_{0} A\right|=\sum_{b \in B}\left|b A \cap b_{0} A\right|-\sum_{b \in B \backslash B_{1}}\left|b A \cap b_{0} A\right| \geqslant \frac{|A||B|}{2 \Delta} .
$$

В частности, $2\left|B_{1}\right| \Delta \geqslant|B|$. Поэтому можно предположить, что $\left|B_{1}\right|>10$, так как в противном случае мы имели бы $\Delta \gg|B|$ и утверждение теоремы сразу следовало бы.

Далее, из неравенств треугольника Ружи следует, что

$$
\left|b A \pm b_{0} A\right| \leqslant \frac{\left|b A-\left(b A \cap b_{0} A\right)\right| \cdot\left|\left(b A \cap b_{0} A\right)-b_{0} A\right|}{\left|b A \cap b_{0} A\right|} \leqslant \frac{|A-A|^{2}}{\left|b A \cap b_{0} A\right|} .
$$


Ввиду (25), имеет место оценка

$$
\left|b A \pm b_{0} A\right| \leqslant \frac{2|A-A|^{2} \Delta}{|A|} \quad \text { для любого } b \in B_{1} .
$$

Для данного $a \in A$ обозначим $a B_{1}(a)=a B_{1} \cap b_{0} A$. Из (26) следует, что

$$
\sum_{a \in A}\left|B_{1}(a)\right|=\sum_{a \in A}\left|a B_{1} \cap b_{0} A\right|=\sum_{b \in B_{1}}\left|b A \cap b_{0} A\right| \geqslant \frac{|A||B|}{2 \Delta} .
$$

Распределяя значения величин $\left|B_{1}(a)\right|$ в двоичные интервалы, получим, что для некоторого $A_{0} \subset A$ и для некоторого числа $N \geqslant 1$ имеют место оценки

$$
\begin{gathered}
N\left|A_{0}\right| \geqslant \frac{|A||B|}{8 \Delta \log |B|}, \\
N \leqslant\left|B_{1}(a)\right| \leqslant 2 N \quad \text { для любого } a \in A_{0} .
\end{gathered}
$$

В частности,

$$
N \geqslant \frac{|B|}{8 \Delta \log |B|} .
$$

Далее, вплоть до неравенства (34), будем пользоваться идеями Бургейна [20]. Заметим, что

$$
\begin{aligned}
\sum_{\left(a, a^{\prime}\right) \in A_{0}^{2}}\left|B_{1}(a) \cap B_{1}\left(a^{\prime}\right)\right| & =\sum_{\left(a, a^{\prime}\right) \in A_{0}^{2}} \sum_{\substack{\lambda \in B_{1}(a) \\
\lambda \in B_{1}\left(a^{\prime}\right)}} 1 \\
& =\sum_{\lambda \in B_{1}}\left(\sum_{\substack{a \in A_{0}: \\
\lambda \in B_{1}(a)}} 1\right)\left(\sum_{\substack{a^{\prime} \in A_{0}: \\
\lambda \in B_{1}\left(a^{\prime}\right)}} 1\right)=\sum_{\lambda \in B_{1}}\left(\sum_{\substack{a \in A_{0}: \\
\lambda \in B_{1}(a)}} 1\right)^{2} .
\end{aligned}
$$

Применяя к сумме по $\lambda \in B_{1}$ неравенство Коши-Буняковского и учитывая, что

$$
\sum_{\lambda \in B_{1}} \sum_{\substack{a \in A_{0}: \\ \lambda \in B_{1}(a)}} 1=\sum_{a \in A_{0}}\left|B_{1}(a)\right| \geqslant N\left|A_{0}\right|
$$

получим

$$
\sum_{\left(a, a^{\prime}\right) \in A_{0}^{2}}\left|B_{1}(a) \cap B_{1}\left(a^{\prime}\right)\right| \geqslant \frac{N^{2}\left|A_{0}\right|^{2}}{\left|B_{1}\right|} .
$$

Распределяя значения величин $\left|B_{1}(a) \cap B_{1}\left(a^{\prime}\right)\right|$ в двоичные интервалы, получим, что для некоторого $G \subset A_{0} \times A_{0}$ и некоторого числа $M \geqslant 1$ выполняется неравенство

$$
M|G| \geqslant \frac{N^{2}\left|A_{0}\right|^{2}}{10\left|B_{1}\right| \log |B|}
$$

и, кроме того, для любого $\left(a, a^{\prime}\right) \in G$

$$
M \leqslant\left|B_{1}(a) \cap B_{1}\left(a^{\prime}\right)\right| \leqslant 2 M .
$$


В частности, так как $|G| \leqslant\left|A_{0}\right|^{2}$, то

$$
M \geqslant \frac{N^{2}}{10\left|B_{1}\right| \log |B|} .
$$

Далее, при $a \in A_{0}$ через $G(a)$ обозначим множество всех элементов $a^{\prime} \in A_{0}$, для которых $\left(a, a^{\prime}\right) \in G$. Пусть

$$
A_{1}=\left\{a \in A_{0}:|G(a)| \geqslant \frac{N^{2}\left|A_{0}\right|}{20 M\left|B_{1}\right| \log |B|}\right\} .
$$

Так как

$$
\sum_{a \in A_{0}}|G(a)|=|G| \geqslant \frac{N^{2}\left|A_{0}\right|^{2}}{10 M\left|B_{1}\right| \log |B|}
$$

то

$$
M\left|A_{1}\right| \geqslant \frac{N^{2}\left|A_{0}\right|}{20\left|B_{1}\right| \log |B|} .
$$

Если $|A|^{5} M^{2} \leqslant 10 N|A||A-A|^{4} \Delta$, то из (31) и (30) получим

$$
|A|^{4}|B|^{3} \ll|A-A|^{4} \Delta^{4}\left|B_{1}\right|^{2} \log ^{5}|B| .
$$

Учитывая, что $B_{1} \subset B,|A-A| \geqslant|A|$, получим

$$
|A-A|^{2} \frac{|A|^{2}|B|^{2}}{E_{\times}(A, B)} \geqslant|A|^{2}|A-A| \Delta \gg|A|^{3} \frac{|B|^{1 / 4}}{(\log |B|)^{5 / 4}},
$$

откуда следует утверждение теоремы в рассматриваемом случае. Поэтому можно предположить, что

$$
|A|^{5} M^{2}>10 N|A||A-A|^{4} \Delta .
$$

Для данного $a_{1} \in A_{1}$ оценим величину $\left|a_{1} B_{1} \pm b_{0} G\left(a_{1}\right)\right|$ при любом фиксированном выборе знака " \pm ". Пусть $\delta \in\{-1,1\}$. Каждому элементу $x \in$ $a_{1} B_{1}+\delta b_{0} G\left(a_{1}\right)$ поставим в соответствие одно представление

$$
x=a_{1} b+\delta b_{0} a_{1}^{\prime}, \quad b \in B_{1}, \quad a_{1}^{\prime} \in G\left(a_{1}\right),
$$

и определим $B_{11}(x)=B_{1}\left(a_{1}\right) \cap B_{1}\left(a_{1}^{\prime}\right)$. Тогда

$$
\delta b_{0}^{2} A+x B_{11}(x) \subset \delta b_{0}^{2} A+b a_{1} B_{1}\left(a_{1}\right)+\delta b_{0} a_{1}^{\prime} B_{1}\left(a^{\prime}\right) \subset b_{0}\left(b A+\delta b_{0} A+\delta b_{0} A\right),
$$

откуда, в силу следствия 2.2 с $k=3$ и оценки (27), имеем

$$
\left|\delta b_{0}^{2} A+x B_{11}(x)\right| \leqslant \frac{\left|b A-\delta b_{0} A\right||A-A|^{2}}{|A|^{2}} \leqslant \frac{2|A-A|^{4} \Delta}{|A|^{3}} .
$$

Поэтому для данного ненулевого элемента $x \in a_{1} B_{1}+\delta b_{0} G\left(a_{1}\right)$ число решений уравнения

$$
b_{0}^{2} a^{\prime}+x b^{\prime}=b_{0}^{2} a^{\prime \prime}+x b^{\prime \prime}, \quad a^{\prime}, a^{\prime \prime} \in A, \quad b^{\prime}, b^{\prime \prime} \in B_{11}(x),
$$


будет удовлетворять условию

$$
\begin{aligned}
E_{+}\left(b_{0}^{2} A, x B_{11}(x)\right) & =E_{+}\left(\delta b_{0}^{2} A, x B_{11}(x)\right) \geqslant \frac{|A|^{2} M^{2}}{\left|\delta b_{0}^{2} A+x B_{11}(x)\right|} \\
& \geqslant \frac{|A|^{2} M^{2}}{\frac{2|A-A|^{4} \Delta}{|A|^{3}}}=\frac{|A|^{5} M^{2}}{2|A-A|^{4} \Delta} .
\end{aligned}
$$

Так как $B_{11}(x) \subset B_{1}\left(a_{1}\right)$, то для каждого ненулевого элемента $x \in a_{1} B_{1}+$ $\delta b_{0} G\left(a_{1}\right)$ число решений уравнения

$$
b_{0}^{2} a^{\prime}+x b^{\prime}=b_{0}^{2} a^{\prime \prime}+x b^{\prime \prime}, \quad a^{\prime}, a^{\prime \prime} \in A, \quad b^{\prime}, b^{\prime \prime} \in B_{1}\left(a_{1}\right),
$$

также будет не меньше чем $|A|^{5} M^{2} /\left(2|A-A|^{4} \Delta\right)$. Следовательно, число решений уравнения

$$
\begin{gathered}
b_{0}^{2} a^{\prime}+x b^{\prime}=b_{0}^{2} a^{\prime \prime}+x b^{\prime \prime}, \\
a^{\prime}, a^{\prime \prime} \in A, \quad b^{\prime}, b^{\prime \prime} \in B_{1}\left(a_{1}\right), \quad x \in a_{1} B_{1}+\delta b_{0} G\left(a_{1}\right) \backslash\{0\},
\end{gathered}
$$

не меньше, чем

$$
\frac{|A|^{5} M^{2}}{2|A-A|^{4} \Delta}\left(\left|a_{1} B_{1}+\delta b_{0} G\left(a_{1}\right)\right|-1\right) \geqslant \frac{|A|^{5} M^{2}}{4|A-A|^{4} \Delta}\left|a_{1} B_{1}+\delta b_{0} G\left(a_{1}\right)\right| .
$$

Но, с другой стороны, это уравнение имеет не более

$$
2 N|A|\left|a_{1} B_{1}+\delta b_{0} G\left(a_{1}\right)\right|+4 N^{2}|A|^{2}
$$

решений (первый член соответствует случаю $b^{\prime}=b^{\prime \prime}$, а второй член соответствует всем остальным наборам $\left(a^{\prime}, a^{\prime \prime}, b^{\prime}, b^{\prime \prime}\right)$, каждый из которых определяет $x$ однозначно). Поэтому

$$
\frac{|A|^{5} M^{2}}{4|A-A|^{4} \Delta}\left|a_{1} B_{1}+\delta b_{0} G\left(a_{1}\right)\right| \leqslant 2 N|A|\left|a_{1} B_{1}+\delta b_{0} G\left(a_{1}\right)\right|+4 N^{2}|A|^{2} .
$$

Учитывая (33), получим

$$
\left|a_{1} B_{1} \pm b_{0} G\left(a_{1}\right)\right| \ll \frac{N^{2}|A-A|^{4} \Delta}{M^{2}|A|^{3}} \quad \text { для любого } a_{1} \in A_{1} .
$$

Согласно (27), для любого $b \in B_{1}$

$$
\left|b A_{1}+b_{0} A_{1}\right| \leqslant \frac{2|A-A|^{2} \Delta}{|A|}=\frac{2|A-A|^{2} \Delta}{|A|\left|A_{1}\right|}\left|A_{1}\right| .
$$

Применяя лемму 3.3 , получим, что для некоторых элементов $a_{1}, a_{11} \in A_{1}$ и $b_{1}, b_{11} \in B_{1}$ либо ${ }^{7}$

$$
\left|\left(a_{1} B_{1}-a_{11} B_{1}\right)+\left(b_{1}-b_{11}\right) A+\left(b_{1}-b_{11}\right) A\right| \gg \frac{\left|A_{1}\right|^{2}\left|B_{1}\right||A|}{|A-A|^{2} \Delta},
$$

\footnotetext{
${ }^{7}$ Лемма 3.3 требует выполнения условий $\left|A_{1}\right| \geqslant 2,\left|B_{1}\right| \geqslant 2$. Условие $\left|B_{1}\right| \geqslant 2$ выполнено, а так как множество $\left(a_{1}-a_{11}\right) B_{1}$ заменяем на множество $a_{1} B_{1}-a_{11} B_{1}$, то выписанное неравенство верно и при $\left|A_{1}\right|=1$.
} 
либо же

$$
\left|\left(a_{1} B_{1}-a_{11} B_{1}\right)+\left(b_{1}-b_{11}\right) A\right| \gg p .
$$

В первом случае, по следствию 2.2 с $k=3$ и $X=-\left(b_{1}-b_{11}\right) A$, получим

$$
\left|\left(a_{1} B_{1}-a_{11} B_{1}\right)-\left(b_{1}-b_{11}\right) A\right| \gg \frac{\left|A_{1}\right|^{2}|A|^{3}\left|B_{1}\right|}{|A-A|^{4} \Delta},
$$

откуда

$$
\left|a_{1} B_{1}-a_{11} B_{1}-b_{1} A+b_{11} A\right| \gg \frac{\left|A_{1}\right|^{2}|A|^{3}\left|B_{1}\right|}{|A-A|^{4} \Delta} .
$$

Снова по следствию 2.2 с $k=4$ и $X=b_{0} A$, а также по неравенству (27), получим

$$
\left|a_{1} B_{1}+b_{0} A\right|\left|a_{11} B_{1}-b_{0} A\right| \gg \frac{\left|A_{1}\right|^{2}|A|^{8}\left|B_{1}\right|}{|A-A|^{8} \Delta^{3}} .
$$

Далее, к множителям в левой части (35) применим неравенства треугольника Ружи. Учитывая, что $G\left(a_{1}\right) \subset A$, будем иметь

$$
\begin{aligned}
\left|a_{1} B_{1}+b_{0} A\right| & \leqslant \frac{\left|a_{1} B_{1}+b_{0} G\left(a_{1}\right)\right|\left|b_{0} G\left(a_{1}\right)-b_{0} A\right|}{\left|G\left(a_{1}\right)\right|} \\
& \leqslant \frac{\left|a_{1} B_{1}+b_{0} G\left(a_{1}\right)\right|\left|b_{0} A-b_{0} A\right|}{\left|G\left(a_{1}\right)\right|}=\frac{\left|a_{1} B_{1}+b_{0} G\left(a_{1}\right)\right||A-A|}{\left|G\left(a_{1}\right)\right|} .
\end{aligned}
$$

Аналогично,

$$
\left|a_{11} B_{1}-b_{0} A\right| \leqslant \frac{\left|a_{11} B_{1}-b_{0} G\left(a_{11}\right)\right||A-A|}{\left|G\left(a_{11}\right)\right|} .
$$

Перемножая эти два неравенства и учитывая (35), получим

$$
\frac{\left|a_{1} B_{1}+b_{0} G\left(a_{1}\right)\right|}{\left|G\left(a_{1}\right)\right|} \frac{\left|a_{11} B_{1}-b_{0} G\left(a_{11}\right)\right|}{\left|G\left(a_{11}\right)\right|} \gg \frac{\left|A_{1}\right|^{2}|A|^{8}\left|B_{1}\right|}{|A-A|^{10} \Delta^{3}} .
$$

Так как $a_{1}, a_{11} \in A_{1}$, то, согласно определению $A_{1}$, будем иметь

$$
\left|G\left(a_{1}\right)\right|\left|G\left(a_{11}\right)\right| \gg \frac{N^{4}\left|A_{0}\right|^{2}}{M^{2}\left|B_{1}\right|^{2}}(\log |B|)^{-2} .
$$

Из последних двух оценок следует, что

$$
\left|a_{1} B_{1}+b_{0} G\left(a_{1}\right)\right|\left|a_{11} B_{1}-b_{0} G\left(a_{11}\right)\right| \gg \frac{N^{4}|A|^{8}\left|A_{1}\right|^{2}\left|A_{0}\right|^{2}}{M^{2}|A-A|^{10}\left|B_{1}\right| \Delta^{3}}(\log |B|)^{-2} .
$$

Учитывая (34), получим

$$
\frac{N^{4}|A-A|^{8} \Delta^{2}}{M^{4}|A|^{6}} \gg \frac{N^{4}|A|^{8}\left|A_{1}\right|^{2}\left|A_{0}\right|^{2}}{M^{2}|A-A|^{10}\left|B_{1}\right| \Delta^{3}}(\log |B|)^{-2} .
$$

Поэтому

$$
|A-A|^{18}\left|B_{1}\right| \Delta^{5} \gg M^{2}|A|^{14}\left|A_{1}\right|^{2}\left|A_{0}\right|^{2}(\log |B|)^{-2} .
$$

Учитывая (32), а затем (28), получим

$$
|A-A|^{18}\left|B_{1}\right|^{3} \Delta^{9} \gg|A|^{18}|B|^{4}(\log |B|)^{-8},
$$

откуда, ввиду $B_{1} \subset B$, следует искомое утверждение. 
Во втором случае имеем

$$
\left|a_{1} B_{1}-a_{11} B_{1}+b_{1} A-b_{11} A\right| \gg p .
$$

Воспользовавшись следствием 2.2 с $k=4$ и $X=b_{0} A$, а также неравенством (27), получим

$$
\left|a_{1} B_{1}+b_{0} A\right|\left|a_{11} B_{1}-b_{0} A\right||A-A|^{4} \Delta^{2} \gg p|A|^{5} .
$$

Рассуждая так же, как в первом случае, и используя неравенства (34), (31), (28) и (30), в итоге получим

$$
|A-A|^{14} \Delta^{8} \gg p|A|^{13}(\log |B|)^{-8} .
$$

Умножая обе части на $|A-A|^{2} \geqslant|A|^{2}$, будем иметь

$$
|A-A|^{2} \frac{|A|^{2}|B|^{2}}{E_{\times}(A, B)} \gg|A|^{3}\left(\frac{p}{|A|}\right)^{1 / 8}(\log |B|)^{-1} .
$$

Так как $p /|A|>(\log |B|)^{200}$, тем самым завершаем доказательство теоремы.

\section{4. Применения к тригонометрическим суммам}

4.1. Формулировка результатов. В настоящем разделе мы применим вариант оценки сумм-произведений разных множеств (теорема 3.1 ) для получения оценок тригонометрических сумм.

ТеОрема 4.1. Пусть $3 \leqslant n \leqslant 1.44 \log \log p-$ натуральное число, $с>0-$ произвольная фиксированная константа. Пусть $X_{1}, X_{2}, \ldots, X_{n}-$ подмножества $\mathbb{F}_{p}^{*}$, удовлетворяющие условию

$$
\left|X_{1}\right| \cdot\left|X_{2}\right| \cdot\left(\left|X_{3}\right| \cdots\left|X_{n}\right|\right)^{1 / 81}>p^{1+c}
$$

Тогда имеет место оценка

$$
\left|\sum_{x_{1} \in X_{1}} \cdots \sum_{x_{n} \in X_{n}} e_{p}\left(x_{1} \cdots x_{n}\right)\right| \ll\left|X_{1}\right| \cdots\left|X_{n}\right| p^{-0.45 c / 2^{n}} .
$$

Так как $n \leqslant 1.44 \log \log p$, то при большом $p$ имеем

$$
p^{-0.45 c / 2^{n}}<e^{-(\log p)^{0.0018}} .
$$

Взяв $n=[1.44 \log \log p], c=0.001$ и используя представление

$$
\sum_{x \in H} e_{p}(a x)=\frac{1}{|H|^{n-1}} \sum_{x_{1} \in a H} \sum_{x_{2} \in H} \cdots \sum_{x_{n} \in H} e_{p}\left(x_{1} x_{2} \cdots x_{n}\right),
$$

из теоремы 4.1 выводим явный вариант результата Бургейна о равномерном распределении маленьких подгрупп мультипликативной группы $\mathbb{F}_{p}^{*}$. 
СлЕДСТВИЕ 4.1. Пусть $Н$ является подгруппой $\mathbb{F}_{p}^{*}$ мощности

$$
|H|>e^{57 \log p / \log \log p} .
$$

Тогда при $p \rightarrow \infty$ имеет место оченка

$$
\max _{(a, p)=1}\left|\sum_{x \in H} e_{p}(a x)\right|=o(|H|) .
$$

Из теоремы 4.1 также получим следующую оценку тригонометрических сумм по степеням первообразного корня $g$ по модулю $p$.

СлЕДСТВИЕ 4.2. Пусть $N>e^{57 \log p / \log \log p}$. Тогда при $p \rightarrow \infty$ имеет место оценка

$$
\max _{(a, p)=1}\left|\sum_{x \leqslant N} e_{p}\left(a g^{x}\right)\right|=o(N) .
$$

Действительно, для любых натуральных чисел $M=o(N)$ и $x_{1}, \ldots, x_{n} \leqslant M$

$$
\sum_{x \leqslant N} e_{p}\left(a g^{x}\right)=\sum_{x \leqslant N} e_{p}\left(a g^{x+x_{1}+\cdots+x_{n}}\right)+O(n M) .
$$

Произведя суммирование по $x_{1}, \ldots, x_{n} \leqslant M$, получим

$$
\sum_{x \leqslant N} e_{p}\left(a g^{x}\right)=\sum_{x \leqslant N}\left\{\frac{1}{M^{n}} \sum_{x_{1} \leqslant M} \cdots \sum_{x_{n} \leqslant M} e_{p}\left(a g^{x} g^{x_{1}} \cdots g^{x_{n}}\right)\right\}+O(n M) .
$$

Взяв $n=[1.44 \log \log p], c=0.001$, выбрав подходящим образом $M$ и применив теорему 4.1, получим следствие 4.2 .

Teоpema 4.2. Пусть $X, Y, Z \subset \mathbb{F}_{p}^{*}$ таковъ, что

$$
|X||Y|>c p, \quad c>0 .
$$

Тогда для любого $\varepsilon>0$ имеет место оценка

$$
\left|\sum_{x \in X} \sum_{y \in Y} \sum_{z \in Z} e_{p}(x y z)\right| \ll|X||Y||Z|^{539 / 540+\varepsilon}
$$

где константа в символе Виноградова может зависеть только от в и с.

На протяжении всего этого раздела будем пользоваться следующей простой оценкой билинейной тригонометрической суммы (которая распространяет оценку (1) на более общий случай).

Лемма 4.1. Пусть $(a, p)=1$. Тогда

$$
\mid \sum_{n=1}^{p} \sum_{m=1}^{p} \nu(n) \varrho(m) e_{p}(\text { anm }) \mid \leqslant \sqrt{p N M}
$$

где $\nu(n), \varrho(m)$ - комплекснье числа и

$$
\sum_{n=1}^{p}|\nu(n)|^{2}=N, \quad \sum_{m=1}^{p}|\varrho(m)|^{2}=M .
$$


Если $X, Y \subset \mathbb{F}_{p}$, то лемма 4.1 влечет оценку

$$
\left|\sum_{x \in X} \sum_{y \in Y} e_{p}(x y)\right| \leqslant \sum_{x \in X}\left|\sum_{y \in Y} e_{p}(x y)\right| \leqslant \sqrt{p|X||Y|} .
$$

Так же, как и оценка (1), лемма 4.1 выводится применением неравенства КошиБуняковского и основных тригонометрических тождеств.

\section{2. Основное утверждение.}

Лемма 4.2. Пусть $X, Y \subset \mathbb{F}_{p}^{*}, s_{1}, \ldots, s_{N} \in \mathbb{F}_{p}^{*} u \delta>0$. Предположим, что для любого $x \in X$ выполняется неравенство

$$
\left|\sum_{y \in Y} \sum_{n=1}^{N} e_{p}\left(x y s_{n}\right)\right| \geqslant \delta|Y| N
$$

Тогда существует подмножество $Z \subset \mathbb{F}_{p}^{*}$ мощности

$$
|Z| \gg|X||Y|^{1 / 81} \delta^{28 / 9}(\log |Y|)^{-1}
$$

такое, что для любого $z \in Z$ имеет место неравенство

$$
\left|\sum_{n=1}^{N} e_{p}\left(z s_{n}\right)\right| \geqslant 0.5 \delta^{2} N
$$

ДокАЗАТЕльство. Справедливость утверждения нетрудно проверить в случае, когда $\delta^{2}<10|X|^{-1}$. Действительно, зафиксируем произвольный элемент $x_{0} \in X$ и определим

$$
Z=\left\{z \in x_{0} Y:\left|\sum_{n=1}^{N} e_{p}\left(z s_{n}\right)\right| \geqslant 0.5 \delta N\right\} .
$$

Тогда $|Z| \geqslant 0.5 \delta|Y|$. Следовательно, в случае $\delta^{2}|X|<10$ имеем

$$
|Z| \geqslant|X||Y| \delta^{3} \geqslant|X||Y|^{1 / 81} \delta^{28 / 9}(\log |Y|)^{-1},
$$

откуда следует, что множество $Z$ удовлетворяет первому требованию утверждения леммы. Второе требование утверждения леммы следует из самого определения множества $Z$.

Таким образом, можно предположить, что $\delta^{2}|X| \geqslant 10$. Предварительно заметим, что для некоторых комплексных чисел $\alpha_{x}$, равных по модулю единице, имеем

$$
\sum_{x \in X} \sum_{y \in Y} \sum_{n=1}^{N} \alpha_{x} e_{p}\left(x y s_{n}\right) \geqslant \delta|X||Y| N .
$$

Следовательно, для некоторого $n=n_{0}$ имеем

$$
\sum_{y \in Y}\left|\sum_{x \in X} \alpha_{x} e_{p}\left(x y s_{n_{0}}\right)\right| \geqslant \delta|X||Y| .
$$

Ввиду леммы 4.1, это влечет, что $\delta^{2}|X||Y| \leqslant p$. 
Из условия леммы следует, что существует элемент $y_{0} \in Y$ такой, что

$$
\sum_{x \in X}\left|\sum_{n=1}^{N} e_{p}\left(x y_{0} s_{n}\right)\right| \geqslant \delta|X| N
$$

Меняя порядки суммирования, получим, что для некоторых комплексных чисел $\alpha_{x}$, равных по модулю единице, имеет место неравенство

$$
\sum_{n=1}^{N}\left|\sum_{x \in X} \alpha_{x} e_{p}\left(x y_{0} s_{n}\right)\right| \geqslant \delta|X| N
$$

Возводя обе части неравенства в квадрат, применяя неравенство Коши-Буняковского по переменной $n$, а затем меняя порядок суммирования, выводим, что

$$
\sum_{x_{1} \in X} \sum_{x_{2} \in X}\left|\sum_{n=1}^{N} e_{p}\left(\left(x_{1}-x_{2}\right) y_{0} s_{n}\right)\right| \geqslant \delta^{2}|X|^{2} N
$$

Так как $\delta^{2}|X| \geqslant 10$, то получим

$$
\sum_{\substack{x_{1} \in X, x_{2} \in X \\ x_{1} \neq x_{2}}}\left|\sum_{n=1}^{N} e_{p}\left(\left(x_{1}-x_{2}\right) y_{0} s_{n}\right)\right| \geqslant 0.9 \delta^{2}|X|^{2} N .
$$

Пусть

$$
E=\left\{\left(x_{1}, x_{2}\right) \in X \times X: x_{1} \neq x_{2},\left|\sum_{n=1}^{N} e_{p}\left(\left(x_{1}-x_{2}\right) y_{0} s_{n}\right)\right| \geqslant 0.5 \delta^{2} N\right\} .
$$

Тогда

$$
|E| \geqslant 0.4 \delta^{2}|X|^{2} \text {. }
$$

Применим лемму 2.4 с $K=3 \delta^{-2}$. Получим, что существует подмножество $X_{1} \subset X$ мощности $\left|X_{1}\right| \geqslant 0.03 \delta^{2}|X|$ такое, что

$$
|X \underset{E}{-} X|^{4} \gg\left|X_{1}-X_{1}\right||X|^{3} \delta^{10} .
$$

Таким образом, полагая $Z_{1}=(X \underset{E}{-} X) y_{0}$, будем иметь

$$
\left|Z_{1}\right|^{4} \gg\left|X_{1}-X_{1}\right||X|^{3} \delta^{10}
$$

Кроме того, по определению, для любого $z \in Z_{1}$ имеем оценку

$$
\left|\sum_{n=1}^{N} e_{p}\left(z s_{n}\right)\right| \geqslant 0.5 \delta^{2} N
$$

Далее, для данного $x \in X_{1}$ определим множество

$$
Y_{x}=\left\{y \in Y:\left|\sum_{n=1}^{N} e_{p}\left(x y s_{n}\right)\right| \geqslant 0.5 \delta N\right\} .
$$


Тогда $\left|Y_{x}\right| \geqslant 0.5 \delta|Y|$. Пусть

$$
Z_{2}=\left\{x y: x \in X_{1}, y \in Y_{x}\right\} .
$$

Так как число пар $(x, y)$ с условиями $x \in X_{1}, y \in Y_{x}$ не меньше $0.5 \delta\left|X_{1}\right||Y|$, а число решений уравнения

$$
x_{1} y_{1}=x_{2} y_{2}, \quad x_{1} \in X_{1}, \quad y_{1} \in Y_{x_{1}}, \quad x_{2} \in X_{1}, \quad y_{2} \in Y_{x_{2}},
$$

не превосходит величины $E_{\times}\left(X_{1}, Y\right)$, то

$$
\left|Z_{2}\right| \geqslant \frac{\delta^{2}\left|X_{1}\right|^{2}|Y|^{2}}{4 E_{\times}\left(X_{1}, Y\right)} .
$$

Кроме того, для любого элемента $z \in Z_{2}$ имеет место оценка

$$
\left|\sum_{n=1}^{N} e_{p}\left(z s_{n}\right)\right| \geqslant 0.5 \delta N \geqslant 0.5 \delta^{2} N
$$

Поэтому достаточно доказать, что одно из множеств $Z_{1}$ или $Z_{2}$ имеет требуемую в утверждении леммы мощность. Из полученных выше оценок величин $\left|Z_{1}\right|$ и $\left|Z_{2}\right|$ следует, что

$$
\left|Z_{1}\right|^{8}\left|Z_{2}\right| \gg\left|X_{1}-X_{1}\right|^{2} \frac{\left|X_{1}\right|^{2}|Y|^{2}}{E_{\times}\left(X_{1}, Y\right)}|X|^{6} \delta^{22} .
$$

Учитывая теорему 3.1 и оценку $\left|X_{1}\right| \geqslant 0.5 \delta^{2}|X|$, получим

$$
\begin{aligned}
\left|Z_{1}\right|^{8}\left|Z_{2}\right| & \gg|X|^{6}\left|X_{1}\right|^{3} \min \left\{|Y|, \frac{p}{\left|X_{1}\right|}\right\}^{1 / 9} \delta^{22}(\log |Y|)^{-1} \\
& \gg|X|^{9} \min \left\{|Y|, \frac{p}{\delta^{2}|X|}\right\}^{1 / 9} \delta^{28}(\log |Y|)^{-1}
\end{aligned}
$$

Так как $\delta^{2} \leqslant p /(|X||Y|)$, то

$$
\left|Z_{1}\right|^{8}\left|Z_{2}\right| \gg|X|^{9}|Y|^{1 / 9} \delta^{28}(\log |Y|)^{-1},
$$

откуда следует утверждение леммы.

Следствие 4.3. Пусть $X, Y \subset \mathbb{F}_{p}^{*}, s_{1}, \ldots, s_{N} \in \mathbb{F}_{p}^{*} u \Delta>0$. Допустим, чmo

$$
\sum_{x \in X}\left|\sum_{y \in Y} \sum_{n=1}^{N} e_{p}\left(x y s_{n}\right)\right| \geqslant \Delta|X||Y| N .
$$

Тогда существует подмножество $Z \subset \mathbb{F}_{p}^{*}$ мощности

$$
|Z| \gg|X||Y|^{1 / 81} \Delta^{28 / 9}(\log |Y|)^{-5}
$$

такое, чmo

$$
\sum_{z \in Z}\left|\sum_{n=1}^{N} e_{p}\left(z s_{n}\right)\right| \geqslant \frac{\Delta^{2}}{100(\log |Y|)^{2}} N|Z| .
$$


ДоказАТЕЛЬСтво. Существует элемент $y_{0} \in Y$ такой, что

$$
\sum_{x \in X}\left|\sum_{n=1}^{N} e_{p}\left(x y_{0} s_{n}\right)\right| \geqslant \Delta|X| N
$$

Следовательно, если $\Delta \leqslant 10|Y|^{-1}$, то, полагая $Z=y_{0} X$, получим требуемое. Поэтому можно предположить, что $\Delta \geqslant 10|Y|^{-1}$. Тогда

$$
\sum_{x \in X}^{\prime}\left|\sum_{y \in Y} \sum_{n=1}^{N} e_{p}\left(x y s_{n}\right)\right| \geqslant 0.9 \Delta|X||Y| N
$$

где штрих над внешним знаком суммирования означает, что оно ведется только по тем $x \in X$, для которых

$$
N \leqslant\left|\sum_{y \in Y} \sum_{n=1}^{N} e_{p}\left(x y s_{n}\right)\right| \leqslant|Y| N
$$

Интервал $[N,|Y| N]$ можно включить в объединение не более чем $2 \log |Y|$ интервалов вида $\left[2^{j-1} N, 2^{j} N\right)$, где $2 \leqslant 2^{j}<2|Y|$. Поэтому существуют подмножество $X_{1} \subset X$ и число $0<\delta \leqslant 1$ такие, что

$$
\delta N|Y| \leqslant\left|\sum_{y \in Y} \sum_{n=1}^{N} e_{p}\left(x y s_{n}\right)\right|<2 \delta N|Y| \quad \text { для всех } x \in X_{1}
$$

и, кроме того,

$$
\delta\left|X_{1}\right| \log |Y| \geqslant 0.2 \Delta|X|
$$

В частности,

$$
\delta \geqslant 0.2 \Delta(\log |Y|)^{-1}, \quad\left|X_{1}\right| \geqslant 0.2 \Delta|X|(\log |Y|)^{-1} .
$$

Из леммы 4.2 (примененной к $X_{1}$ вместо $X$ ) получим, что существует подмножество $Z \subset \mathbb{F}_{p}^{*}$ мощности

$$
|Z| \gg\left|X_{1}\right||Y|^{1 / 81} \delta^{28 / 9}(\log |Y|)^{-1} \gg|X||Y|^{1 / 81} \Delta^{28 / 9}(\log |Y|)^{-5}
$$

такое, что для всех $z \in Z$ имеют место неравенства

$$
\left|\sum_{n=1}^{N} e_{p}\left(z s_{n}\right)\right| \geqslant 0.5 \delta^{2} N \geqslant \frac{\Delta^{2}}{100(\log |Y|)^{2}} N .
$$

Суммируя это неравенство по множеству $z \in Z$, завершаем доказательство следствия.

4.3. Вывод оценок полилинейных тригонометрических сумм. Докажем теорему 4.1. Заменяя в следствии 4.3 величину $\Delta$ на $\Delta(\log p)^{3}$, для больших $p$ получим следующее утверждение, которое имеет удобную для итерации форму. 
Пусть $X_{1}, X_{2}, \ldots, X_{n}$ являются подмножествами $\mathbb{F}_{p}^{*}, \Delta>0$. Предположим, что

$$
\frac{1}{\left|X_{1}\right|\left|X_{2}\right| \cdots\left|X_{n}\right|(\log p)^{3}} \sum_{x_{1} \in X_{1}}\left|\sum_{x_{2} \in X_{2}} \cdots \sum_{x_{n} \in X_{n}} e_{p}\left(x_{1} x_{2} \cdots x_{n}\right)\right| \geqslant \Delta .
$$

Тогда существует множество $X_{11} \subset \mathbb{F}_{p}^{*}$ мощности

$$
\left|X_{11}\right| \geqslant\left|X_{1}\right|\left|X_{n}\right|^{1 / 81} \Delta^{28 / 9}
$$

такое, что

$$
\frac{1}{\left|X_{11}\right|\left|X_{2}\right| \cdots\left|X_{n-1}\right|(\log p)^{3}} \sum_{x_{1} \in X_{11}}\left|\sum_{x_{2} \in X_{2}} \cdots \sum_{x_{n-1} \in X_{n-1}} e_{p}\left(x_{1} x_{2} \cdots x_{n-1}\right)\right| \geqslant \Delta^{2} .
$$

В частности, после каждой итерации нижняя оценка соответствующей тригонометрической суммы изменится с $\Delta$ на $\Delta^{2}$. Таким образом, после $(n-2)$ шагов придем к некоторому множеству $X_{1}^{\prime}$ мощности

$$
\left|X_{1}^{\prime}\right| \geqslant\left|X_{1}\right|\left(\left|X_{n}\right| \cdots\left|X_{3}\right|\right)^{1 / 81} \Delta^{28 / 9+2 \cdot 28 / 9+\cdots+2^{n-3} \cdot 28 / 9}
$$

такое, что

$$
\frac{1}{\left|X_{1}^{\prime}\right|\left|X_{2}\right|(\log p)^{3}} \sum_{x_{1} \in X_{1}^{\prime}}\left|\sum_{x_{2} \in X_{2}} e_{p}\left(x_{1} x_{2}\right)\right| \geqslant \Delta^{2^{n-2}} .
$$

Последнее неравенство и лемма 4.1 влекут

$$
\Delta^{2^{n-1}} \leqslant \frac{p}{\left|X_{1}^{\prime}\right|\left|X_{2}\right|}
$$

Так как

$$
\left|X_{1}^{\prime}\right|\left|X_{2}\right| \geqslant\left|X_{1}\right|\left|X_{2}\right|\left(\left|X_{n}\right| \cdots\left|X_{3}\right|\right)^{1 / 81} \Delta^{(28 / 9)\left(2^{n-2}-1\right)} \geqslant p^{1+c} \Delta^{(28 / 9)\left(2^{n-2}-1\right)},
$$

то

$$
\Delta^{2^{n+1}} \leqslant p^{-c}
$$

Следовательно, учитывая, что $n \leqslant 1.44 \log \log p$, для достаточно большого $p$ имеем

$$
\left(\Delta(\log p)^{3}\right)^{2^{n+1}} \leqslant p^{-c}(\log p)^{2^{n+3}} \leqslant p^{-0.9 c} .
$$

Таким образом, получаем неравенство

$$
\Delta(\log p)^{3} \leqslant p^{-0.9 c / 2^{n+1}},
$$

которое завершает доказательство теоремы 4.1.

Теперь докажем теорему 4.2. Пусть

$$
\sum_{x \in X}\left|\sum_{y \in Y} \sum_{z \in Z} e_{p}(x y z)\right|=|X||Y||Z| \Delta .
$$


Можно предположить, что $\Delta \geqslant 10|Z|^{-1}$ (в противном случае утверждение тривиально). Аналогично доказательству следствия 4.3, множество элементов $x \in X$, для которых

$$
|Y| \leqslant\left|\sum_{y \in Y} \sum_{z \in Z} e_{p}(x y z)\right| \leqslant|Y||Z|
$$

включаем в объединение не более чем $2 \log |Z|$ подмножеств и находим подмножество $X_{1} \subset X$ и число $0<\delta \leqslant 1$ такие, что

$$
\delta|Y||Z| \leqslant\left|\sum_{y \in Y} \sum_{z \in Z} e_{p}(x y z)\right|<2 \delta|Y||Z| \quad \text { для всех } x \in X_{1}
$$

и, кроме того, $\delta\left|X_{1}\right| \log |Z| \gg \Delta|X|$. В частности,

$$
\delta \gg \Delta(\log |Z|)^{-1}, \quad\left|X_{1}\right| \gg \Delta|X|(\log |Z|)^{-1} .
$$

Так как

$$
\sum_{x \in X_{1}} \sum_{y \in Y}\left|\sum_{z \in Z} e_{p}(x y z)\right| \geqslant \delta\left|X_{1}\right||Y||Z|,
$$

то, фиксируя нужным образом элемент $y=y_{0}$ и применяя лемму 4.1 , предварительно получим, что $\delta^{2}\left|X_{1}\right||Z| \leqslant p$.

Из (36) следует, что для некоторого $z_{0} \in Z$ имеет место оценка

$$
\sum_{x \in X_{1}}\left|\sum_{y \in Y} e_{p}\left(x y z_{0}\right)\right| \geqslant \delta\left|X_{1}\right||Y| .
$$

Будем следовать идеям доказательства леммы 4.2. Меняя в последнем неравенстве порядок суммирования (с вводом комплексных коэффициентов $\alpha_{x}$, по модулю равных единице), применяя неравенство Коши-Буняковского по переменной $y$, а затем еще раз меняя порядки суммирования, получим

$$
\sum_{\left(x_{1}, x_{2}\right) \in X_{1}^{2}}\left|\sum_{y \in Y} e_{p}\left(\left(x_{1}-x_{2}\right) z_{0} y\right)\right| \geqslant \delta^{2}|Y|\left|X_{1}\right|^{2} .
$$

Поэтому множество

$$
E=\left\{\left(x_{1}, x_{2}\right) \in X_{1} \times X_{1}:\left|\sum_{y \in Y} e_{p}\left(\left(x_{1}-x_{2}\right) z_{0} y\right)\right| \geqslant 0.5 \delta^{2}|Y|\right\}
$$

имеет мощность

$$
|E| \geqslant 0.5 \delta^{2}\left|X_{1}\right|^{2}
$$

Так как для любого $t \in X_{1} \underset{E}{-} X_{1}$ выполняется оценка

$$
\left|\sum_{y \in Y} e_{p}\left(t z_{0} y\right)\right|^{2} \gg \delta^{4}|Y|^{2},
$$


TO

$$
\delta^{4}|Y|^{2}\left|X_{1}-X_{E}\right| \ll \sum_{t \in \mathbb{F}_{p}}\left|\sum_{y \in Y} e_{p}\left(t z_{0} y\right)\right|^{2}=p|Y| .
$$

Применяя лемму 2.4 с $K=2 \delta^{-2}$, получим, что существует подмножество $X_{1}^{\prime} \subset X_{1}$ мощности $\left|X_{1}^{\prime}\right| \gg \delta^{2}\left|X_{1}\right|$ такое, что

$$
\left|X_{1} \underset{E}{-} X_{1}\right|^{4} \gg\left|X_{1}^{\prime}-X_{1}^{\prime}\right|\left|X_{1}\right|^{3} \delta^{10}
$$

Следовательно,

$$
p^{4} \gg|Y|^{4}\left|X_{1}\right|^{3}\left|X_{1}^{\prime}-X_{1}^{\prime}\right| \delta^{26}
$$

Далее, имеем

$$
\sum_{x \in X_{1}^{\prime}}\left|\sum_{y \in Y} \sum_{z \in Z} e_{p}(x y z)\right| \geqslant \delta\left|X_{1}^{\prime}\right||Y||Z| .
$$

Таким образом, для некоторых коэффициентов $\alpha_{x}$, равных по модулю единице, имеет место неравенство

$$
\sum_{y \in Y}\left|\sum_{x \in X_{1}^{\prime}} \sum_{z \in Z} \alpha_{x} e_{p}(x y z)\right| \geqslant \delta\left|X_{1}^{\prime}\right||Y||Z| .
$$

Применяя к сумме по переменной $y$ неравенство Коши-Буняковского, а затем распространяя суммирование по $y \in Y$ до полной системы $y \in \mathbb{F}_{p}$, выводим, что

$$
p E_{\times}\left(X_{1}^{\prime}, Z\right) \gg \delta^{2}\left|X_{1}^{\prime}\right|^{2}|Y||Z|^{2} .
$$

Учитывая неравенство (38) и используя теорему 3.1 , получим

$$
p^{9} \gg|Y|^{9}\left|X_{1}\right|^{6} \delta^{54}\left|X_{1}^{\prime}\right|^{3} \min \left\{|Z|, \frac{p}{\left|X_{1}^{\prime}\right|}\right\}^{1 / 9}(\log |Z|)^{-1} .
$$

Так как $\left|X_{1}^{\prime}\right| \gg \delta^{2}\left|X_{1}\right|$, то будем иметь

$$
p^{9} \gg \delta^{60}|Y|^{9}\left|X_{1}\right|^{9} \min \left\{|Z|, \frac{p}{\delta^{2}\left|X_{1}\right|}\right\}^{1 / 9}(\log |Z|)^{-1} .
$$

Вспоминая, что

$$
\delta^{2} \leqslant \frac{p}{\left|X_{1}\right||Z|}, \quad \delta\left|X_{1}\right| \log |Z| \gg|X| \Delta
$$

а также учитывая неравенства (37), получим $\Delta<|Z|^{-1 / 540+\varepsilon}$. Требуемое доказано.

4.4. Тригонометрические суммы Морделла. Напомним, что известная оценка Вейля [41] (см. также [42]) тригонометрической суммы утверждает, что если

$$
f(x)=\sum_{i=1}^{n} a_{i} x^{i} \in \mathbb{Z}[x], \quad\left(a_{n}, p\right)=1,
$$


то выполнено неравенство

$$
\left|\sum_{x=0}^{p-1} e_{p}(f(x))\right| \leqslant n p^{1 / 2}
$$

Так же как и в случае сумм Гаусса, эта оценка нетривиальна при $n<p^{1 / 2}$. Тригонометрической суммой Морделла называется сумма вида

$$
\sum_{x=0}^{p-1} e_{p}\left(a_{1} x^{n_{1}}+\cdots+a_{r} x^{n_{r}}\right), \quad\left(a_{i}, p\right)=1 .
$$

При этом $r$ считается фиксированной константой. Эти суммы были исследованы Морделлом [43] еще до появления работы Вейля [41].

Результаты Морделла были улучшены в работах [44], [45] (на основе метода Степанова). В [46] Бургейн, основываясь на варианте сумм-произведений для подмножеств $\mathbb{F}_{p} \times \mathbb{F}_{p}$, получил следующую оценку тригонометрических сумм Морделла (см. также работы [25] и [47], где приведен ряд аналогичных результатов).

ТЕОРема 4.3. Для данного натурального числа $r$ и положительной константы $\varepsilon>0$ существует $\delta=\delta(r, \varepsilon)>0$ такое, что если

$$
\begin{aligned}
\left(n_{i}, p-1\right) & <p^{1-\varepsilon} & \text { для всех } 1 \leqslant i \leqslant r, \\
\left(n_{i}-n_{j}, p-1\right) & <p^{1-\varepsilon} & \text { для всех } 1 \leqslant i \neq j \leqslant r,
\end{aligned}
$$

то имеет место оченка

$$
\left|\sum_{x=0}^{p-1} e_{p}\left(a_{1} x^{n_{1}}+\cdots+a_{r} x^{n_{r}}\right)\right|<p^{1-\delta} .
$$

При $r=1$ сумма Морделла является суммой Гаусса, поэтому результат вытекает из следствия 4.1. При $r=2$ имеем сумму

$$
S=\sum_{x=1}^{p-1} e_{p}\left(a_{1} x^{n_{1}}+a_{2} x^{n_{2}}\right) .
$$

Заметим, что если $\left(x_{1}, \ldots, x_{s}\right) \in\left(\mathbb{F}_{p}^{*}\right)^{s}$, то

$$
\sum_{x=1}^{p-1} e_{p}\left(a_{1} x^{n_{1}}+a_{2} x^{n_{2}}\right)=\sum_{x=1}^{p-1} e_{p}\left(a_{1} x_{1}^{n_{1}} \cdots x_{s}^{n_{1}} x^{n_{1}}+a_{2} x_{1}^{n_{2}} \cdots x_{s}^{n_{2}} x^{n_{2}}\right) .
$$

Применение идей, использованных при оценке полилинейной тригонометрической суммы, приводит к необходимости изучения проблемы суммы и произведения для подмножеств кольца $\mathbb{F}_{p} \times \mathbb{F}_{p}$. Если $\left(a_{1}, a_{2}\right) \in \mathbb{F}_{p} \times \mathbb{F}_{p},\left(a_{3}, a_{4}\right) \in \mathbb{F}_{p} \times \mathbb{F}_{p}$, то в этом кольце операции суммы и произведения определяются как

$$
\left(a_{1}, a_{2}\right)+\left(a_{3}, a_{4}\right)=\left(a_{1}+a_{3}, a_{2}+a_{4}\right), \quad\left(a_{1}, a_{2}\right)\left(a_{3}, a_{4}\right)=\left(a_{1} a_{3}, a_{2} a_{4}\right) .
$$

По аналогии с одномерным случаем, если $A$ является подмножеством $\mathbb{F}_{p} \times \mathbb{F}_{p}$ мощности близкой к $\left|\mathbb{F}_{p} \times \mathbb{F}_{p}\right|=p^{2}$, то оба множества $A+A$ и $A A$ будут иметь 
мощности близкие к мощности множества $A$. Кроме этого имеются еще следующие исключительные случаи. Если существует элемент $a \in \mathbb{F}_{p}$ такой, что множество $A$ в некотором смысле будет близким к одному из множеств

$$
\{a\} \times \mathbb{F}_{p}, \quad \mathbb{F}_{p} \times\{a\}, \quad\left\{(x, a x): x \in \mathbb{F}_{p}\right\},
$$

то мощности множеств $A+A$ и $A A$ также будут близки к мощности множества $A$. Бургейн [46] доказал, что эти случаи, грубо говоря, являются единственными исключениями.

ПРЕДЛОЖЕНИЕ 4.1. Пусть подмножество $A \subset \mathbb{F}_{p} \times \mathbb{F}_{p}$ имеет мощность $|A|>p^{\varepsilon_{0}}$, где $\varepsilon_{0}>0$ - некоторая фиксированная константа. Предположим, чmo

$$
|A+A|+|A A|<p^{\varepsilon}|A| .
$$

Тогда для некоторого $\varepsilon^{\prime}, \varepsilon^{\prime}=\varepsilon^{\prime}(\varepsilon) \rightarrow 0$ при $\varepsilon \rightarrow 0$, имеет место один из следующих случаев:

(i) $|A|>p^{2-\varepsilon^{\prime}}$;

(ii) существует элемент $a \in \mathbb{F}_{p}$ такой, что либо

$$
\left|A \cap\left(\{a\} \times \mathbb{F}_{p}\right)\right|>p^{-\varepsilon^{\prime}}|A|,
$$

либо же

$$
\left|A \cap\left(\mathbb{F}_{p} \times\{a\}\right)\right|>p^{-\varepsilon^{\prime}}|A| ;
$$

(iii) существует элемент $a \in \mathbb{F}_{p}$ такой, что

$$
\left|A \cap\left\{(x, a x): x \in \mathbb{F}_{p}\right\}\right|>p^{-\varepsilon^{\prime}}|A| .
$$

Более того, в случаях (ii), (iii)

$$
p^{1-\varepsilon^{\prime}}<|A|<p^{1+\varepsilon^{\prime}} .
$$

Заметим, что если выполняется (ii) или (iii), то очевидно, что $|A|<p^{1+\varepsilon^{\prime}}$. Применив оценку сумм-произведений в $\mathbb{F}_{p}$ и заменив при необходимости $\varepsilon^{\prime}$ на $\varepsilon^{\prime \prime}=\varepsilon^{\prime \prime}(\varepsilon) \rightarrow 0$, легко также добиться выполнения условия $|A|>p^{1-\varepsilon^{\prime}}$.

Оценка сумм-произведений в произвольных кольцах получена в недавней работе Тао [48]. Докажем предложение 4.1, следуя схеме доказательства оценки сумм-произведений в простых полях (теорема 1.1). Ниже $c_{1}, \ldots, c_{6}$ обозначают некоторые положительные константы.

Достаточно рассмотреть случай $A \subset \mathbb{F}_{p}^{*} \times \mathbb{F}_{p}^{*}$. Заметим, что элемент $\left(a_{1}, a_{2}\right) \in$ $\mathbb{F}_{p} \times \mathbb{F}_{p}$ обратим тогда и только тогда, когда обе координаты $a_{1}$ и $a_{2}$ отличны от нулевого элемента поля $\mathbb{F}_{p}$.

Будем рассуждать от противного. Предположим, что существует константа $c_{1}=\varepsilon^{\prime}$ такая, что, каким бы маленьким ни было $\varepsilon$, ни одно из условий (i), (ii), (iii) не выполняется.

Следуя доказательству теоремы 1.1 , для некоторого $y_{0} \in A$ будем иметь

$$
\sum_{x \in A}\left|x A \cap y_{0} A\right| \geqslant \frac{|A|^{3}}{|A A|} .
$$


Поэтому, полагая $A_{1}=\left\{x \in A:\left|x A \cap y_{0} A\right| \geqslant|A|^{2} /(2|A A|)\right\}$, получим, что

$$
\left|A_{1}\right| \geqslant \frac{|A|^{2}}{2|A A|}>0.5|A| p^{-\varepsilon},
$$

причем

$$
\left|x A \cap y_{0} A\right| \geqslant \frac{|A|^{2}}{2|A A|}>0.5|A| p^{-\varepsilon} \quad \text { при всех } x \in A_{1} .
$$

По неравенствам треугольника Ружи имеем

$$
\left|x A \pm y_{0} A\right| \leqslant 4|A| p^{-3 \varepsilon} .
$$

Из невыполнения условия (ii) нетрудно вывести, что найдется подмножество $B \subset A_{1}$ с условием $p^{c_{2}}>|B|>p^{c_{3}}$ такое, что попарно различные элементы множества $B$ будут иметь попарно различные первые и попарно различные вторые координаты. Так как

$$
|A-A| \leqslant \frac{|A+A|^{2}}{|A|} \leqslant|A| p^{2 \varepsilon},
$$

то можно считать, что $|A-A||B-B|<p^{2-c_{4}}$. Так как ненулевые элементы множества $B-B$ обратимы, то для любого $\xi \notin\left(A_{1}-A_{1}\right) /(B-B)$ имеет место равенство

$$
\left|\xi B+A_{1}\right|=\left|A_{1}\right||B| .
$$

Зафиксируем произвольный элемент $x_{0} \in A_{1}$. В силу невыполнения условия (iii), можно найти элементы $\left(z_{1}, z_{1}^{\prime}\right) \in A_{1},\left(z_{2}, z_{2}^{\prime}\right) \in A_{1}$ такие, что $z_{1} / z_{1}^{\prime} \neq$ $z_{2} / z_{2}^{\prime}$. Таким образом,

$$
\left\{n \frac{\left(z_{1}, z_{1}^{\prime}\right)}{x_{0}}+m \frac{\left(z_{2}, z_{2}^{\prime}\right)}{x_{0}}:(n, m) \in \mathbb{Z} \times \mathbb{Z}\right\}=\mathbb{F}_{p} \times \mathbb{F}_{p} .
$$

Поэтому при некотором $x \in A_{1}$ будем иметь

$$
\frac{A_{1}-A_{1}}{B-B}+\frac{x}{x_{0}} \notin \frac{A_{1}-A_{1}}{B-B} .
$$

Итак, существуют элементы $x_{1}, x_{2} \in A_{1}, x_{3}, x_{4} \in B$ с условием $x_{3} \neq x_{4}$ такие, что

$$
\frac{x_{1}-x_{2}}{x_{3}-x_{4}}+\frac{x}{x_{0}} \notin \frac{A_{1}-A_{1}}{B-B} .
$$

Следовательно,

$$
\left|\left(\frac{x_{1}-x_{2}}{x_{3}-x_{4}}+\frac{x}{x_{0}}\right) B+A_{1}\right|=\left|A_{1}\right||B| .
$$

Таким образом,

$$
\left|\left(x_{1}-x_{2}\right) x_{0} B+\left(x_{3}-x_{4}\right) x B+\left(x_{3}-x_{4}\right) x_{0} A_{1}\right| \geqslant|A| p^{c_{5}} .
$$

Из этого неравенства следует, что

$$
\left|x_{1} x_{0} A-x_{2} x_{0} A+x_{3} x A-x_{4} x A+x_{3} x_{0} A-x_{4} x_{0} A\right| \geqslant|A| p^{c_{5}} .
$$


Применяя неравенство Ружи-Плюнеке (следствие 2.2 с $k=6$ и $X=y_{0}^{2} A$ ) и неравенства треугольника Ружи, получим, что для некоторых $x_{5}, x_{6} \in A_{1}$ выполняется оценка

$$
\left|x_{5} x_{6} A+y_{0}^{2} A\right| \geqslant|A| p^{c_{6}} .
$$

Снова применяя неравенство треугольника Ружи (следствие 2.2 с $k=2$ и $X=$ $\left.x_{5} y_{0} A\right)$, приходим к противоречию с неравенством (39). Предложение 4.1 доказано.

При $r \geqslant 3$ задача оценки суммы Морделла сводится к случаям $r=1$ и $r=2$. Поясним это для $r=3$. Требуется нетривиально оценить сумму

$$
W:=\sum_{x=0}^{p-1} e_{p}\left(a x^{n}+b x^{m}+c x^{k}\right),
$$

где $a, b, c, n, m, k$ удовлетворяют соответствующим условиям теоремы 4.3. Пусть $J_{s}$ обозначает число решений системы сравнений

$$
\left\{\begin{array}{l}
x_{1}^{m}+\cdots+x_{s}^{m} \equiv x_{s+1}^{m}+\cdots+x_{2 s}^{m}(\bmod p), \\
x_{1}^{k}+\cdots+x_{s}^{k} \equiv x_{s+1}^{k}+\cdots+x_{2 s}^{k}(\bmod p)
\end{array}\right.
$$

в целых числах $0 \leqslant x_{1}, \ldots, x_{2 s} \leqslant p-1$. Представляя $J_{s}$ в виде двойной тригонометрической суммы, получим

$$
J_{s}=\frac{1}{p^{2}} \sum_{a=0}^{p-1} \sum_{b=0}^{p-1}\left|\sum_{x=0}^{p-1} e_{p}\left(a x^{m}+b x^{k}\right)\right|^{2 s} .
$$

Выделяя член, соответствующий $a=b=0$, получим

$$
J_{s}=p^{2 s-2}+\frac{1}{p^{2}} \sum_{\substack{(a, b) \in \mathbb{F}_{p}^{2} \\(a, b) \neq(0,0)}}\left|\sum_{x=0}^{p-1} e_{p}\left(a x^{m}+b x^{k}\right)\right|^{2 s} .
$$

Согласно предположению, при каждом $(a, b) \neq(0,0)$ сумма по $x$ оценивается сверху величиной $p^{1-\delta}$, где $\delta=\delta(\varepsilon)>0$. Поэтому

$$
J_{s}=p^{2 s-2}+\theta p^{2 s(1-\delta)}, \quad|\theta| \leqslant 1 .
$$

В частности, при $s>1 / \delta$ получим $J_{s} \leqslant 2 p^{2 s-2}$.

Теперь заметим, что для любого $y \not \equiv 0(\bmod p)$ выполняется равенство

$$
W=\sum_{x=0}^{p-1} e_{p}\left(a y^{n} x^{n}+b y^{m} x^{m}+c y^{k} x^{k}\right) .
$$

Следовательно,

$$
p|W|^{2 s} \leqslant \sum_{y=0}^{p-1}\left|\sum_{x=0}^{p-1} e_{p}\left(a y^{n} x^{n}+b y^{m} x^{m}+c y^{k} x^{k}\right)\right|^{2 s} .
$$


Отсюда имеем

$$
\begin{gathered}
p|W|^{2 s} \leqslant \sum_{x_{1}=0}^{p-1} \cdots \sum_{x_{2 s}=0}^{p-1} \sum_{y=0}^{p-1} e_{p}\left(a y^{n}\left(x_{1}^{n}+\cdots+x_{s}^{n}-x_{s+1}^{n} \cdots-x_{2 s}^{n}\right)\right. \\
\quad+b y^{m}\left(x_{1}^{m}+\cdots+x_{s}^{m}-x_{s+1}^{m} \cdots-x_{2 s}^{m}\right) \\
\left.\quad+c y^{k}\left(x_{1}^{k}+\cdots+x_{s}^{k}-x_{s+1}^{k} \cdots-x_{2 s}^{k}\right)\right) .
\end{gathered}
$$

Применяя неравенство Гёльдера по переменным $x_{1}, \ldots, x_{2 s}$, получим

$$
p^{2 s}|W|^{4 s^{2}} \leqslant p^{2 s(2 s-1)} \sum_{\lambda=0}^{p-1} \sum_{\mu=0}^{p-1} \sum_{\nu=0}^{p-1} I(\lambda, \mu, \nu)\left|\sum_{y=0}^{p-1} e_{p}\left(a \lambda y^{n}+b \mu y^{m}+c \nu y^{k}\right)\right|^{2 s}
$$

где $I(\lambda, \mu, \nu)$ обозначает число решений системы сравнений

$$
\left\{\begin{array}{l}
x_{1}^{n}+\cdots+x_{s}^{n} \equiv x_{s+1}^{n}+\cdots+x_{2 s}^{n}+\lambda(\bmod p) \\
x_{1}^{m}+\cdots+x_{s}^{m} \equiv x_{s+1}^{m}+\cdots+x_{2 s}^{m}+\mu(\bmod p) \\
x_{1}^{k}+\cdots+x_{s}^{k} \equiv x_{s+1}^{k}+\cdots+x_{2 s}^{k}+\nu(\bmod p)
\end{array}\right.
$$

в целых числах $0 \leqslant x_{1}, \ldots, x_{2 s} \leqslant p-1$. Из представления

$$
I(\lambda, \mu, \nu)=\frac{1}{p^{3}} \sum_{a=0}^{p-1} \sum_{b=0}^{p-1} \sum_{c=0}^{p-1}\left|\sum_{x=0}^{p-1} e_{p}\left(a x^{n}+b x^{m}+c x^{k}\right)\right|^{2 s} e_{p}(-a \lambda-b \mu-c \nu)
$$

следует, что $I(\lambda, \mu, \nu) \leqslant I(0,0,0)$. Поэтому

$$
\begin{aligned}
p^{2 s}|W|^{4 s^{2}} & \leqslant p^{2 s(2 s-1)} I(0,0,0) \sum_{\lambda=0}^{p-1} \sum_{\mu=0}^{p-1} \sum_{\nu=0}^{p-1}\left|\sum_{y=0}^{p-1} e_{p}\left(a \lambda y^{n}+b \mu y^{m}+c \nu y^{k}\right)\right|^{2 s} \\
& =p^{2 s(2 s-1)+3} I(0,0,0)^{2} .
\end{aligned}
$$

Так как $I(0,0,0) \leqslant J_{s} \leqslant 2 p^{2 s-2}$, получим

$$
p^{2 s}|W|^{4 s^{2}} \ll p^{2 s(2 s-1)+3+4 s-4} .
$$

Следовательно,

$$
|W| \ll p^{1-\delta^{\prime}}, \quad \delta^{\prime}=\frac{1}{4 s^{2}} .
$$

Оценка сумм-произведений в $\mathbb{F}_{p} \times \mathbb{F}_{p}$ была применена Бургейном [49] также для оценки тригонометрической суммы

$$
W=W(a, b, c ; \lambda)=\sum_{x=1}^{t} \sum_{y=1}^{t} e_{p}\left(a \lambda^{x}+b \lambda^{y}+c \lambda^{x y}\right), \quad(c, p)=1
$$

где $\lambda$ - элемент поля $\mathbb{F}_{p}$ мультипликативного порядка $t$ (мультипликативный порядок $\lambda$ - это минимальное натуральное число $n$ такое, что $\lambda^{n}=1$ ). Такие суммы были изучены в работах [50], [51], а затем и в работах [52]-[55]. 
Нетривиальные оценки этой суммы влекут свойства равномерного распределения тройки

$$
\left\{\left(\lambda^{x}, \lambda^{y}, \lambda^{x y}\right): 1 \leqslant x, y \leqslant t\right\} \subset \mathbb{F}_{p}^{3}
$$

В работе [54] была получена нетривиальная (и явная) оценка при $t>p^{1 / 2+\varepsilon}$. Бургейн [49] получил нетривиальную оценку при $t>p^{\varepsilon}$ для любой константы $\varepsilon>0$. Чанг и Яо [56] получили явную оценку при $t>p^{1 / 4+\varepsilon}$. Из работы [57] известно, что нетривиальные оценки $W$ имеют интересные приложения в установлении свойств равномерного распределения чисел Мерсенна $M_{n}=2^{q_{n}}-1$ по модулю $p$, где $q_{n}$ обозначает $n$-е простое число. Альтернативные методы (основанные на методе большого решета Линника) по распределению чисел Мерсенна и более общих экспоненциальных последовательностей можно найти в работах [58] и [59].

4.5. Случай составных модулей. Нетривиальные обобщения результатов по оценкам сумм-произведений множеств на случай колец $\mathbb{Z}_{q}$, где $q$ - не обязательно простое число, и их приложения к оценкам тригонометрических сумм в $\mathbb{Z}_{q}$ были получены Бургейном и Чанг [60] для $q$, имеющих не очень много простых делителей, и Бургейном [61]-[63] для произвольных $q$.

Сформулируем несколько результатов Бургейна. Для $A \subset \mathbb{Z}_{q}$ и для делителя $q_{1} \mid q$, пусть $\pi_{q_{1}}(A)$ является проекцией множества $A$ на кольцо $\mathbb{Z}_{q_{1}}$.

ТеОремА 4.4. Для любых положительных констант $\delta_{1}, \delta_{2}$ существуют положительные константы $\varepsilon, \delta_{3}$ такие, что справедливо следующее утверждение. Пусть $A \subset \mathbb{Z}_{q}$ удовлетворяет условиям

(i) $|A|<q^{1-\delta_{1}}$;

(ii) если $q_{1} \mid q$ и $q_{1}>q^{\varepsilon}$, mо $\left|\pi_{q_{1}}(A)\right|>q_{1}^{\delta_{2}}$. Тогда имеет место оценка

$$
|A+A|+|A A|>q^{\delta_{3}}|A| \text {. }
$$

ТЕОРемА 4.5. Для любой константы $\gamma>0$ существуют $\varepsilon>0, \tau>0$ и положительное целое число $k$ такие, что имеет место следующее утверждение. Пусть $A_{1}, \ldots, A_{k} \subset \mathbb{Z}_{q}$ таковы, что если $q_{1} \mid q, q_{1}>q^{\varepsilon}, \xi \in \mathbb{Z}_{q_{1}}$ то

$$
\left|A_{i} \cap \pi_{q_{1}}^{-1}(\xi)\right|<q_{1}^{-\gamma}\left|A_{i}\right|, \quad i=1, \ldots, k .
$$

Тогда

$$
\max _{a \in \mathbb{Z}_{q}^{*}}\left|\sum_{x_{1} \in A_{1}} \cdots \sum_{x_{k} \in A_{k}} e_{q}\left(a x_{1} \cdots x_{k}\right)\right|<q^{-\tau}\left|A_{1}\right| \cdots\left|A_{k}\right| .
$$

ТеОРема 4.6. Пусть Н является мультипликативной подгруппой группъ $\mathbb{Z}_{q}^{*}$ мощности $|H|>q^{\varepsilon}$, где $\varepsilon>0$. Тогда

$$
\max _{a \in \mathbb{Z}_{q}^{*}}\left|\sum_{x \in H} e_{q}(a x)\right|<q^{-\delta}|H|, \quad \delta=\delta(\varepsilon)>0 .
$$




\section{5. $\mathbb{F}_{p}$-аналог теоремы Семереди-Троттера}

Теорема Семереди-Троттера [64] утверждает, что если $\mathscr{P}$ является конечным множеством точек $(x, y) \in \mathbb{R}^{2}$ и если $\mathscr{L}$ является конечным множеством прямых $\ell \subset \mathbb{R}^{2}$, то имеет место следующая оценка для числа инцидентов "точка-прямая":

$$
W(\mathscr{P}, \mathscr{L}):=\#\{((x, y), \ell) \in \mathscr{P} \times \mathscr{L}:(x, y) \in \ell\} \ll|\mathscr{P}|+|\mathscr{L}|+(|\mathscr{P}||\mathscr{L}|)^{2 / 3} .
$$

Элекеш [65] обнаружил оригинальное применение теоремы Семереди-Троттера к проблеме сумм-произведений подмножеств множества действительных чисел $\mathbb{R}$. Пусть $A$ - конечное множество ненулевых действительных чисел. Элекеш рассмотрел множество точек

$$
\mathscr{P}=\{(x, y): x \in A+A, y \in A A\}
$$

и множество прямых $\mathscr{L}=\{\ell=\ell(z, t): z \in A, t \in A\}$, где $\ell(z, t)$ задается уравнением

$$
y-z x+z t=0
$$

В частности,

$$
|\mathscr{P}|=|A A||A+A|, \quad|\mathscr{L}|=|A|^{2} .
$$

Каждая прямая $\ell(z, t)$ содержит не меньше $|A|$ различных точек

$$
(x, y)=(a+t, a z) \in \mathscr{P}, \quad a \in A .
$$

Таким образом, $W(\mathscr{P}, \mathscr{L}) \geqslant|A||\mathscr{L}|=|A|^{3}$. Сопоставляя эту оценку с оценкой Семереди-Троттера, получим

$$
|A|^{3} \ll|A A||A+A|^{2}+|A|^{2}+\left(|A A||A+A||A|^{2}\right)^{2 / 3} .
$$

Отсюда следует известная оценка Элекеша для сумм-произведений конечных множеств действительных чисел: $\max \{|A+A|,|A A|\} \gg|A|^{5 / 4}$. Как мы уже отмечали, к настоящему моменту наилучший результат в этой проблеме принадлежит Шолимоши: $\max \{|A+A|,|A A|\} \gg|A|^{4 / 3-\varepsilon}$.

Приведем еще один пример из недавней работы [66], где показано, что для конечных непустых множеств $A \subset \mathbb{R}$ имеет место оценка $|A(A+1)| \gg|A|^{5 / 4}$. Можно предположить, что $\{0,-1\} \notin A \subset \mathbb{R}$. Положим

$$
\mathscr{P}=\{(x, y) \in A(A+1) \times A(A+1)\}
$$

и определим множество прямых $\mathscr{L}=\{\ell=\ell(z, t): z \in A, t \in A+1\}$ уравнением

$$
y-\frac{z}{t} x-z=0
$$

В частности,

$$
|\mathscr{P}|=|A(A+1)|^{2}, \quad|\mathscr{L}|=|A|^{2} .
$$

Каждая прямая $\ell(z, t) \in \mathscr{L}$ содержит не меньше $|A|$ различных точек $(x, y) \in \mathscr{P}$, определяемых как

$$
(x, y)=(a t,(a+1) z), \quad a \in A .
$$

Поэтому $W(\mathscr{P}, \mathscr{L}) \geqslant|A|^{3}$. Следовательно, применив оценку Семереди-Троттера, получим, что $|A(A+1)| \gg|A|^{5 / 4}$. 
5.1. Аналог теоремы Семереди-Троттера. Доказательство теоремы Семереди-Троттера в $\mathbb{R}^{2}$ использует упорядоченность множества действительных чисел, поэтому оно не проходит в случае $\mathbb{F}_{p}^{2}$. Бургейн, Кац и Тао [6] показали, что, используя оценку сумм-произведений подмножеств $\mathbb{F}_{p}$, можно получить следующий $\mathbb{F}_{p}$-аналог теоремы Семереди-Троттера.

Теорема 5.1. Пусть Я (соответственно $\mathscr{L}$ ) - некоторое множество точек (соответственно прямых) на плоскости $\mathbb{F}_{p} \times \mathbb{F}_{p}$. Пусть для некоторой положительной константы $\alpha<2$ выполняется условие

$$
\max \{|\mathscr{P}|,|\mathscr{L}|\} \leqslant M<p^{\alpha} .
$$

Тогда для некоторой константы $\gamma=\gamma(\alpha)>0$ имеет место оценка

$$
W(\mathscr{P}, \mathscr{L}):=\#\{((x, y), \ell) \in \mathscr{P} \times \mathscr{L}:(x, y) \in \ell\} \ll M^{3 / 2-\gamma} .
$$

Легко доказать, что $W(\mathscr{P}, \mathscr{L}) \ll|\mathscr{P}|^{1 / 2}|\mathscr{L}|+|\mathscr{P}|$. Действительно, по неравенству Коши-Буняковского,

$$
\begin{aligned}
& \#\left\{\left((x, y), \ell, \ell^{\prime}\right) \in \mathscr{P} \times \mathscr{L} \times \mathscr{L}:(x, y) \in \ell \cap \ell^{\prime}\right\} \\
& \quad=\sum_{(x, y) \in \mathscr{P}}|\{\ell \in \mathscr{L}:(x, y) \in \ell\}|^{2} \geqslant \frac{1}{|\mathscr{P}|}(W(\mathscr{P}, \mathscr{L}))^{2} .
\end{aligned}
$$

С другой стороны, случай $\ell=\ell^{\prime}$ вносит в левую часть величину $W(\mathscr{P}, \mathscr{L})$, в то время как случай $\ell \neq \ell^{\prime}$ вносит величину, не превосходящую $|\mathscr{L}|^{2}$ (ввиду того, что $\left.\left|\ell \cap \ell^{\prime}\right| \leqslant 1\right)$. Таким образом, левая часть не превосходит величины

$$
W(\mathscr{P}, \mathscr{L})+|\mathscr{L}|^{2},
$$

откуда следует, что

$$
W(\mathscr{P}, \mathscr{L}) \ll|\mathscr{P}|^{1 / 2}|\mathscr{L}|+|\mathscr{P}| .
$$

В частности, если $|\mathscr{P}| \leqslant M,|\mathscr{L}| \leqslant M$, то получаем (тривиальную) оценку $W(\mathscr{P}, \mathscr{L}) \leqslant M^{3 / 2}$. Без дополнительных предположений эта оценка неулучшаема. Действительно, если взять $M=p^{2}, \mathscr{P}=\mathbb{F}_{p}^{2}$ и $\mathscr{L}$ - множество всех прямых в $\mathbb{F}_{p}^{2}$, то на каждой прямой будет лежать $p$ точек. Поэтому получим $W(\mathscr{P}, \mathscr{L}) \gg M^{3 / 2}$. Таким образом, естественно предположить, что $M<p^{\alpha}$ для некоторой положительной константы $\alpha<2$. Теорема 5.1 утверждает, что при этом предположении имеет место нетривиальная оценка $W(\mathscr{P}, \mathscr{L}) \leqslant$ $M^{3 / 2-\gamma}$. Заметим, что для больших $M$ имеет место более точная оценка

$$
W(\mathscr{P}, \mathscr{L}) \ll \frac{M^{2}}{p}+p^{1 / 2} M
$$

полученная в работе Винха [67].

Некоторые моменты вывода теоремы 5.1 из оценки сумм-произведений мы обсудим в следующем пункте. 
5.2. Растягивающиеся отображения от двух переменных. Из оценки сумм-произведений и неравенств треугольника Ружи легко следует, что многочлен $f(x, y, z)=x y+z: \mathbb{F}_{p}^{3} \rightarrow \mathbb{F}_{p}$ обладает следующим свойством растяжения: для любых подмножеств $A, B, C \subset \mathbb{F}_{p}$ с условием

$$
|A|=|B|=|C|=N \sim p^{\alpha},
$$

где $\alpha<1$ - положительная константа, мощность множества

$$
f(A, B, C)=\{a b+c: a \in A, b \in B, c \in C\}
$$

не меньше $N^{1+\beta}$ для некоторой константы $\beta=\beta(\alpha)>0$. Действительно, пусть $b_{0}$ является ненулевым элементом множества $B$. Тогда из неравенств треугольника Ружи имеем

$$
|A+A|=\left|A b_{0}+A b_{0}\right| \leqslant \frac{\left|A b_{0}+C\right|^{2}}{|C|} \leqslant \frac{|A B+C|^{2}}{N} .
$$

Кроме того, из мультипликативной формы неравенств треугольника Ружи имеем

$$
|A A| \leqslant \frac{|A B|^{2}}{|B|} \leqslant \frac{|A B+C|^{2}}{N} .
$$

Соединяя последние два неравенства с оценкой сумм-произведений, получим

$$
|A B+C| \geqslant N \max \{|A+A|,|A A|\}>N^{1+\beta}, \quad \beta=\beta(\alpha)>0 .
$$

Ввиду важных приложений растягивающихся отображений, Вигдерсон поставил задачу (см. сноску в [68; с. 2] или [69; с. 237]) выписать явную функцию от двух переменных, которая удовлетворяла бы свойству растяжения. Другими словами, требуется найти функцию $f(x, y)$ такую, что для любых подмножеств $A, B \subset \mathbb{F}_{p}$ с условием $|A|=|B|=N \sim p^{\alpha}$, где $\alpha<1$ - положительная константа, выполняется оценка

$$
|\{f(a, b): a \in A, b \in B\}| \geqslant N^{1+\beta}
$$

для некоторой константы $\beta=\beta(\alpha)>0$. Эта задача была решена Бургейном [68]. Используя $\mathbb{F}_{p}$-аналог теоремы Семереди-Троттера, он показал, что, например, многочлен $f(x, y)=x^{2}+x y$ удовлетворяет требуемому свойству растяжения (см. также [70] и [71; гл. 8]).

Теорема 5.2. Пусть $A, B$ - подмножества поля $\mathbb{F}_{p}$ такие, что $|A|=$ $|B|=N<p^{\alpha}$, где $\alpha<1$ - положительная константа. Тогда для некоторой константы $\beta=\beta(\alpha)>0$ имеет место оценка

$$
\left|\left\{a^{2}+a b: a \in A, b \in B\right\}\right| \geqslant N^{1+\beta} .
$$

ДокАзАтельство. Можно предположить, что $\{0\} \notin A \cup B$.

Положим $C=\{a(a+b): a \in A, b \in B\}$. В качестве множества точек возьмем множество $\mathscr{P}=C \times C$. В частности, $|\mathscr{P}|=|C|^{2}$. Рассмотрим множество прямых

$$
\mathscr{L}=\left\{y=\frac{a_{2}}{a_{1}} x+\left(a_{2}^{2}-a_{1} a_{2}\right): a_{1}, a_{2} \in A\right\} .
$$


Ясно, что $N^{2} \ll|\mathscr{L}| \leqslant N^{2} \leqslant|C|^{2}$. Каждая прямая $y=\left(a_{2} / a_{1}\right) x+\left(a_{2}^{2}-a_{1} a_{2}\right)$ содержит как минимум следующие $N$ точек:

$$
\left\{(x, y)=\left(a_{1}\left(a_{1}+b\right), a_{2}\left(a_{2}+b\right)\right) \in \mathscr{P}: b \in B\right\} .
$$

Таким образом, $W(\mathscr{P}, \mathscr{L}) \gg N^{3}$. Поэтому из $\mathbb{F}_{p}$-аналога теоремы СемередиТроттера следует, что $N^{3} \leqslant\left(|C|^{2}\right)^{3 / 2-\gamma}$, откуда получаем требуемое утверждение. Теорема доказана.

Вывод самого аналога теоремы Семереди-Троттера основан на идее работы Каца и Тао [72]. Предполагая противное, можно доказать, что существуют некоторые множества $X, Y$ мощности порядка $N^{1 / 2}$ такие, что

$$
\left|\left\{\left(x_{1}, x_{2}, y\right)\right\} \in X \times X \times Y:(1-y) x_{1}+y x_{2} \in X, y \neq 0,1\right| \geqslant N^{3 / 2-o(1)} .
$$

Затем, с помощью оценки типа Балога-Семереди-Гауэрса, показывается, что последнее неравенство противоречит оценке сумм-произведений подмножеств $\mathbb{F}_{p}$. Эти идеи применительно к множеству $C=\{a(a+b): a \in A, b \in B\}$ будут выглядеть приблизительно следующим образом. Предположим, что $|C|<N^{1+o(1)}$. Пусть вектор $\left(a_{1}, a_{2}, b_{1}, b_{2}, b_{3}\right) \in A^{2} \times B^{3}$ таков, что элементы $b_{1}$, $b_{2}, b_{3}-$ попарно различные. Рассматривая на прямой

$$
y=\frac{a_{2}}{a_{1}} x+\left(a_{2}^{2}-a_{1} a_{2}\right)
$$

три точки

$$
\left(x_{i}, y_{i}\right)=\left(a_{1}^{2}+a_{1} b_{i}, a_{2}^{2}+a_{2} b_{i}\right), \quad i=1,2,3,
$$

получим соотношение

$$
x_{2}\left(y_{3}-y_{1}\right)+x_{3}\left(y_{1}-y_{2}\right)=x_{1}\left(y_{3}-y_{2}\right) .
$$

Так как имеется порядка $N^{5}$ векторов $\left(a_{1}, a_{2}, b_{1}, b_{2}, b_{3}\right) \in A^{2} \times B^{3}$ с попарно различными элементами $b_{1}, b_{2}, b_{3}$, то легко видеть, что имеется по меньшей мере порядка $N^{5}$ векторов $\left(x_{1}, x_{2}, x_{3}, y_{1}, y_{2}, y_{3}\right) \in C^{6}$, удовлетворяющих уравнению (40) и таких, что элементы $y_{1}, y_{2}, y_{3}$ являются попарно различными. Поэтому можно зафиксировать $y_{2}=y_{0}, y_{3}=y_{0}^{\prime}$ таким образом, что

$\#\left\{\left(x_{2}, x_{3}, y_{1}\right) \in C^{3}: y_{1} \neq y_{0}, y_{0}^{\prime}, x_{2}\left(y_{0}^{\prime}-y_{1}\right)+x_{3}\left(y_{1}-y_{0}\right) \in\left(y_{0}^{\prime}-y_{0}\right) C\right\}>N^{3-o(1)}$.

Отсюда легко вывести, что существует подмножество $C^{\prime} \subset C$ порядка $\left|C^{\prime}\right|>$ $N^{1-o(1)}$ такое, что для любого $x_{3} \in C^{\prime}$ выполняется неравенство

$$
\#\left\{\left(x_{2}, y_{1}\right) \in C^{2}: y_{1} \neq y_{0}, y_{0}^{\prime}, x_{2}\left(y_{0}^{\prime}-y_{1}\right)+x_{3}\left(y_{1}-y_{0}\right) \in\left(y_{0}^{\prime}-y_{0}\right) C\right\}>N^{2-o(1)} .
$$

В частности, суммируя это неравенство по множеству $x_{3} \in C^{\prime}$, получим, что для некоторого фиксированного $y_{1}=y_{0}^{\prime \prime} \in C$, отличного от $y_{0}, y_{0}^{\prime}$, имеет место оценка

$\#\left\{\left(x_{2}, x_{3}\right) \in C \times C^{\prime}: y_{1} \neq y_{0}, y_{0}^{\prime}, x_{2}\left(y_{0}^{\prime}-y_{0}^{\prime \prime}\right)+x_{3}\left(y_{0}^{\prime \prime}-y_{0}\right) \in\left(y_{0}^{\prime}-y_{0}\right) C\right\}>N^{2-o(1)}$. 
Применяя оценку Балога-Семереди-Гауэрса получим, что существует подмножество $C^{\prime \prime} \subset C^{\prime}$ мощности $\left|C^{\prime \prime}\right|>N^{1-o(1)}$ такое, что

$$
\left|C^{\prime \prime}+C^{\prime \prime}\right|=\left|\left(y_{0}^{\prime \prime}-y_{0}\right) C^{\prime \prime}+\left(y_{0}^{\prime \prime}-y_{0}\right) C^{\prime \prime}\right|<N^{1+o(1)} .
$$

Далее, неравенство (41) справедливо для любого $x_{3} \in C^{\prime}$, в частности, и для любого $x_{3} \in C^{\prime \prime}$. Поэтому, суммируя (41) по всем $x_{3} \in C^{\prime \prime}$, получим, что для некоторого фиксированного $x_{2}=x_{0} \in C$ имеет место оценка

$$
\#\left\{\left(x_{3}, y_{1}\right) \in C^{\prime \prime} \times C: x_{0}\left(y_{0}^{\prime}-y_{1}\right)+x_{3}\left(y_{1}-y_{0}\right) \in\left(y_{0}^{\prime}-y_{0}\right) C\right\}>N^{2-o(1)} .
$$

Итак,

$$
\#\left\{\left(x_{3}, y_{1}\right) \in C^{\prime \prime} \times C:\left(x_{3}-x_{0}\right)\left(y_{1}-y_{0}\right) \in\left(y_{0}^{\prime}-y_{0}\right) C+x_{0} y_{0}-x_{0} y_{0}^{\prime}\right\}>N^{2-o(1)} .
$$

Применяя оценку Балога-Семереди-Гауэрса в мультипликативной форме, получим, что для некоторого подмножества $C^{\prime \prime \prime} \subset C^{\prime \prime}$ мощности $\left|C^{\prime \prime \prime}\right|>N^{1-o(1)}$ имеет место оценка

$$
\left|\left(C^{\prime \prime \prime}-x_{0}\right)\left(C^{\prime \prime \prime}-x_{0}\right)\right|<N^{1+o(1)} .
$$

Так как

$$
\left|\left(C^{\prime \prime \prime}-x_{0}\right)+\left(C^{\prime \prime \prime}-x_{0}\right)\right|=\left|C^{\prime \prime \prime}+C^{\prime \prime \prime}\right| \leqslant\left|C^{\prime \prime}+C^{\prime \prime}\right| \leqslant N^{1+o(1)},
$$

то последние два неравенства противоречат оценке сумм-произведений.

Касаясь еще раз явных примеров растягивающихся отображений, можно рассматривать более общий случай

$$
f(x, y)=q_{1}(x)+q_{2}(x) y: \mathbb{F}_{p} \times \mathbb{F}_{p} \rightarrow \mathbb{F}_{p},
$$

где $q_{1}(x), q_{2}(x)$ - многочлены такие, что $q_{1}(x) / q_{2}(x)$ не является константой. Можно положить $\mathscr{P}=C \times C$, где $C=\left\{q_{1}(a)+q_{2}(a) b: a \in A, b \in B\right\}$, а в качестве множества прямых можно взять

$$
\mathscr{L}=\left\{y=\frac{q_{2}\left(a_{2}\right)}{q_{2}\left(a_{1}\right)} x+q_{1}\left(a_{2}\right)-\frac{q_{2}\left(a_{2}\right)}{q_{2}\left(a_{1}\right)} q_{1}\left(a_{1}\right): a_{1}, a_{2} \in A\right\} .
$$

При этом каждая прямая

$$
y=\frac{q_{2}\left(a_{2}\right)}{q_{1}\left(a_{1}\right)} x+q_{1}\left(a_{2}\right)-\frac{q_{2}\left(a_{2}\right)}{q_{2}\left(a_{1}\right)} q_{1}\left(a_{1}\right)
$$

будет содержать точки вида

$$
(x, y)=\left(q_{1}\left(a_{1}\right)+q_{2}\left(a_{1}\right) b, q_{1}\left(a_{2}\right)+q_{2}\left(a_{2}\right) b\right) \in \mathscr{P}, \quad b \in B .
$$

5.3. Оценки некоторых двойных тригонометрических сумм. С помощью $\mathbb{F}_{p}$-аналога теоремы Семереди-Троттера, Бургейн [68] получил новые оценки некоторых важных двойных тригонометрических сумм. Приведем один из таких результатов. 
ТеОрема 5.3. Пусть $A, B$ - подмножества поля $\mathbb{F}_{p}$ такие, что $|A|<p^{1 / 2}$, $|B|<p^{1 / 2}$. Тогда

$$
\max _{(a, p)=1}\left|\sum_{x \in A} \sum_{y \in B} e_{p}\left(a\left(x y+x^{2} y^{2}\right)\right)\right|<p^{1-\gamma},
$$

где $\gamma>0$ - абсолютная константа.

Из теоремы 5.3 можно вывести, что существуют абсолютные константы $\rho>0$ и $\gamma>0$ такие, что для любых подмножеств $A, B \subset \mathbb{F}_{p}$ мощностей $|A|>p^{1 / 2-\rho}$, $|B|>p^{1 / 2-\rho}$ имеет место оценка

$$
\left|\sum_{x \in A} \sum_{y \in B} \operatorname{sign} \sin \left(\frac{2 \pi}{p}\left(x y+x^{2} y^{2}\right)\right)\right|<p^{-\gamma}|A||B| .
$$

Таким образом, наряду с оценками двойных тригонометрических сумм, Бургейн получил важные приложения $\mathbb{F}_{p}$-аналога теоремы Семереди-Троттера в теории экстракторов. С проблемами, связанными с экстракторами, можно подробно ознакомиться по работам [68], [70], [73]-[75].

Следуя работе Бургейна, покажем, как применяется аналог теоремы Семереди-Троттера, на примере доказательства теоремы 5.3. По неравенству Гёльдера,

$$
\begin{aligned}
& \left|\sum_{x \in A} \sum_{y \in B} e_{p}\left(a\left(x y+x^{2} y^{2}\right)\right)\right|^{3} \\
& \leqslant|A|^{2} \sum_{x \in A}\left|\sum_{y_{1}, y_{2}, y_{3} \in B} e_{p}\left(a x\left(y_{1}+y_{2}+y_{3}\right)+a x^{2}\left(y_{1}^{2}+y_{2}^{2}+y_{3}^{2}\right)\right)\right| \\
& \quad=|A|^{2} \sum_{y_{1}, y_{2}, y_{3} \in B} \sum_{x \in A} \alpha_{x} e_{p}\left(a x\left(y_{1}+y_{2}+y_{3}\right)+a x^{2}\left(y_{1}^{2}+y_{2}^{2}+y_{3}^{2}\right)\right),
\end{aligned}
$$

где $\alpha_{x}$ - комплексные числа, равные по модулю единице. Еще раз применяя неравенство Гёльдера, получим, что

$$
\begin{aligned}
& \left|\sum_{x \in A} \sum_{y \in B} e_{p}\left(a\left(x y+x^{2} y^{2}\right)\right)\right|^{9} \\
& \quad \leqslant|A|^{6}|B|^{6} \sum_{y_{1}, y_{2}, y_{3} \in B}\left|\sum_{x \in A} \alpha_{x} e_{p}\left(a x\left(y_{1}+y_{2}+y_{3}\right)+a x^{2}\left(y_{1}^{2}+y_{2}^{2}+y_{3}^{2}\right)\right)\right|^{3} .
\end{aligned}
$$

Пусть $I_{B}\left(\mu_{1}, \mu_{2}\right)$ - число решений системы уравнений

$$
\left\{\begin{array}{l}
y_{1}+y_{2}+y_{3}=\mu_{1} \\
y_{1}^{2}+y_{2}^{2}+y_{3}^{2}=\mu_{2}
\end{array}\right.
$$

в переменных $y_{1}, y_{2}, y_{3} \in B$. Тогда

$$
\begin{aligned}
& \left|\sum_{x \in A} \sum_{y \in B} e_{p}\left(a\left(x y+x^{2} y^{2}\right)\right)\right|^{9} \\
& \quad \leqslant|A|^{6}|B|^{6} \sum_{\mu_{1} \in \mathbb{F}_{p}} \sum_{\mu_{2} \in \mathbb{F}_{p}} I_{B}\left(\mu_{1}, \mu_{2}\right)\left|\sum_{x \in A} \alpha_{x} e_{p}\left(a x \mu_{1}+a x^{2} \mu_{2}\right)\right|^{3} .
\end{aligned}
$$


Пусть $J(A)$ - число решений системы сравнений

$$
\left\{\begin{array}{l}
x_{1}+x_{2}+x_{3}=x_{4}+x_{5}+x_{6} \\
x_{1}^{2}+x_{2}^{2}+x_{3}^{2}=x_{4}^{2}+x_{5}^{2}+x_{6}^{2}
\end{array}\right.
$$

в переменных $x_{1}, \ldots, x_{6} \in A$, а $J(B)$ - число решений системы сравнений

$$
\left\{\begin{array}{l}
y_{1}+y_{2}+y_{3}=y_{4}+y_{5}+y_{6} \\
y_{1}^{2}+y_{2}^{2}+y_{3}^{2}=y_{4}^{2}+y_{5}^{2}+y_{6}^{2}
\end{array}\right.
$$

в переменных $y_{1}, \ldots, y_{6} \in B$. Так как

$$
\begin{gathered}
\sum_{\mu_{1} \in \mathbb{F}_{p}} \sum_{\mu_{2} \in \mathbb{F}_{p}} I_{B}\left(\mu_{1}, \mu_{2}\right)^{2}=J(B), \\
\sum_{\mu_{1} \in \mathbb{F}_{p}} \sum_{\mu_{2} \in \mathbb{F}_{p}}\left|\sum_{x \in A} \alpha_{x} e_{p}\left(a x \mu_{1}+a x^{2} \mu_{2}\right)\right|^{6} \leqslant p^{2} J(A),
\end{gathered}
$$

то, возведя обе части (42) в квадрат и применяя неравенство Коши-Буняковского по переменным $\mu_{1}, \mu_{2}$, получим

$$
\left|\sum_{x \in A} \sum_{y \in B} e_{p}\left(a\left(x y+x^{2} y^{2}\right)\right)\right|^{18} \leqslant p^{2}|A|^{12}|B|^{12} J(A) J(B) \ll p^{14} J(A) J(B) .
$$

Таким образом, достаточно доказать, что для некоторой абсолютной положительной константы $c$ имеют место оценки

$$
J(A) \ll p^{2-c}, \quad J(B) \ll p^{2-c}
$$

(новизна как раз и состоит в доказательстве этих оценок). Очевидно, что величина $J(A)$ равна числу решений сравнения

$$
\left\{\begin{array}{l}
x_{1}+x_{2}+x_{3}=x_{4}+x_{5}+x_{6} \\
x_{1} x_{2}+x_{2} x_{3}+x_{3} x_{1}=x_{4} x_{5}+x_{5} x_{6}+x_{6} x_{4}
\end{array}\right.
$$

в переменных $x_{1}, \ldots, x_{6} \in A$. Поэтому

$$
J(A)=\sum_{\lambda_{1} \in \mathbb{F}_{p}} \sum_{\lambda_{2} \in \mathbb{F}_{p}} I\left(\lambda_{1}, \lambda_{2}\right)^{2}
$$

где $I\left(\lambda_{1}, \lambda_{2}\right)$ обозначает число решений системы уравнений

$$
\left\{\begin{array}{l}
x_{1}+x_{2}+x_{3}=\lambda_{1} \\
x_{1} x_{2}+x_{2} x_{3}+x_{3} x_{1}=\lambda_{2}
\end{array}\right.
$$

в переменных $x_{1}, x_{2}, x_{3} \in A$. Пусть $\varepsilon>0$ - очень маленькая абсолютная константа (в несколько раз меньшая, чем константа $\gamma$, которая присутствует в формулировке аналога теоремы Семереди-Троттера, и соответствующая значению, скажем, $\alpha=3 / 2)$. Пусть

$$
G=\left\{\left(\lambda_{1}, \lambda_{2}\right): I\left(\lambda_{1}, \lambda_{2}\right)>p^{1 / 2-\varepsilon}\right\}
$$


Так как

$$
\sum_{\left(\lambda_{1}, \lambda_{2}\right) \notin G} I\left(\lambda_{1}, \lambda_{2}\right)^{2} \leqslant p^{1 / 2-\varepsilon} \sum_{\lambda_{1} \in \mathbb{F}_{p}} \sum_{\lambda_{2} \in \mathbb{F}_{p}} I\left(\lambda_{1}, \lambda_{2}\right)=p^{1 / 2-\varepsilon}|A|^{3} \ll p^{2-\varepsilon},
$$

TO

$$
J(A) \ll \sum_{\left(\lambda_{1}, \lambda_{2}\right) \in G} I\left(\lambda_{1}, \lambda_{2}\right)^{2}+p^{2-\varepsilon} .
$$

Далее, так как

$$
|G| p^{1 / 2-\varepsilon} \leqslant \sum_{\left(\lambda_{1}, \lambda_{2}\right) \in G} I\left(\lambda_{1}, \lambda_{2}\right) \leqslant \sum_{\lambda_{1} \in \mathbb{F}_{p}} \sum_{\lambda_{2} \in \mathbb{F}_{p}} I\left(\lambda_{1}, \lambda_{2}\right)=|A|^{3} \ll p^{3 / 2},
$$

то $|G| \ll p^{1+\varepsilon}$. Из (43) и определения множества $G$ получим, что число решений уравнения

$$
\lambda_{2}=\left(x_{1}+x_{2}\right) \lambda_{1}-\left(x_{1}^{2}+x_{1} x_{2}+x_{2}^{2}\right), \quad\left(\lambda_{1}, \lambda_{2}\right) \in G, \quad x_{1} \in A, \quad x_{2} \in A,
$$

не меньше, чем $|G| p^{1 / 2-\varepsilon}$. Теперь рассмотрим семейство прямых

$$
\mathscr{L}=\left\{\xi-\eta\left(x_{1}+x_{2}\right)+\left(x_{1}^{2}+x_{1} x_{2}+x_{2}^{2}\right)=0: x_{1} \in A, x_{2} \in A\right\}
$$

и множество точек $\mathscr{P}=G$. Ясно, что

$$
|\mathscr{L}| \approx|A|^{2} \ll p, \quad|\mathscr{P}|=|G|
$$

Кроме того,

$$
\left\{\left(\left(\lambda_{2}, \lambda_{1}\right), \ell\right) \in \mathscr{P} \times \mathscr{L}:\left(\lambda_{2}, \lambda_{1}\right) \in \ell\right\} \gg|G| p^{1 / 2-\varepsilon} .
$$

Так как

$$
|\mathscr{P}|+|\mathscr{L}| \ll p+|G|
$$

то применив $\mathbb{F}_{p}$-аналог теоремы Семереди-Троттера, получим, что

$$
|G| p^{1 / 2-\varepsilon} \ll(p+|G|)^{3 / 2-\gamma},
$$

где $\gamma>0$ - константа, которая присутствует в формулировке $\mathbb{F}_{p}$-аналога теоремы Семереди-Троттера, соответствующая значению $\alpha=3 / 2$. Так как $|G| \ll p^{1+\varepsilon}$ и величина $\varepsilon$ значительно меньше, чем $\gamma$, получим

$$
|G| \ll p^{1-0.5 \gamma}
$$

Наконец, используя тривиальную оценку $I\left(\lambda_{1}, \lambda_{2}\right) \ll|A|$, получим

$$
J(A) \ll p^{1-0.5 \gamma}|A|^{2}+p^{2-\varepsilon} \ll p^{2-c}, \quad c>0 .
$$

Аналогично имеем $J(B) \ll p^{2-c}$. Тем самым теорема доказана. 
5.4. Заключение. В настоящей статье мы привели в основном примеры применения оценок сумм-произведений только к оценкам рациональных тригонометрических сумм. Но оценки сумм-произведений нашли также ряд других важных применений. Одно из таких применений содержится в работе Хельфготта [76], где доказано, что если $A$ является подмножеством группы $\mathrm{SL}_{2}\left(\mathbb{F}_{p}\right)$, не содержащимся ни в одной собственной подгруппе, и если $|A|<p^{3-\varepsilon}$ для некоторого $\varepsilon>0$, то имеет место оценка

$$
|A A A| \gg|A|^{1+\delta}, \quad \delta=\delta(\varepsilon)>0 .
$$

Затем Хельфготт вывел, что если $A$ является множеством генераторов группы $\mathrm{SL}_{2}\left(\mathbb{F}_{p}\right)$ (т.е. $A$ такое, что каждый элемент $g \in \mathrm{SL}_{2}\left(\mathbb{F}_{p}\right)$ может быть представлен в виде произведения элементов из $A \cup A^{-1}$ ), то диаметр графа Кэли $\Gamma\left(\mathrm{SL}_{2}\left(\mathbb{F}_{p}\right), A\right)$ не превосходит величины $c_{1}(\log p)^{c_{2}}$, где $c_{1}, c_{2}$ - абсолютные константы. Недавно Хельфготт в [77] получил аналог этого результата и для группы $\mathrm{SL}_{3}\left(\mathbb{F}_{p}\right)$.

Бургейн и Гамбурд [78], [79], основываясь на работах Хельфготта, получили новые результаты по семействам экспандеров. С экспандерами и их приложениями можно ознакомиться по монографии [80], а также по работам [81], [82].

С помощью оценок сумм-произведений Чанг [83] получила новые оценки сумм характеров по специальным множествам. ${ }^{8}$ Ряд интересных задач, касающихся проблемы сумм-произведений и их применений, можно найти в работе Чанг [85].

Автор выражает благодарность С. В. Конягину за ряд ценных замечаний, а также А. Вигдерсону за сообщение о работе [70].

\section{Список литературы}

[1] P. Erdös, E. Szeméredi, "On sums and products of integers", Studies in pure mathematics, Birkhäuser, Basel, 1983, 213-218.

[2] J. Solymosi, "Bounding multiplicative energy by the sumset", Adv. Math., 222:2 (2009), 402-408.

[3] T. Tao, Structure and randomness, Pages from year one of a mathematical blog, Amer. Math. Soc., Providence, RI, 2008, ISBN: 978-0-8218-4695-7, xii+298 pp.

[4] Z. Dvir, "On the size of Kakeya sets in finite fields", J. Amer. Math. Soc., 22:4 (2009), 1093-1097.

[5] J. Bourgain, A. A. Glibichuk, S. V. Konyagin, "Estimates for the number of sums and products and for exponential sums in fields of prime order", J. London Math. Soc. (2), 73:2 (2006), 380-398.

[6] J. Bourgain, N. Katz, T. Tao, "A sum-product estimate in finite fields, and applications", Geom. Funct. Anal., 14:1 (2004), 27-57.

[7] С. А. Степанов, "О числе точек гиперэллиптической кривой над простым конечным полем", Изв. АН СССР. Сер. матем., 33:5 (1969), 1171-1181; англ. пер.: S. A. Stepanov, "On the number of points of a hyperelliptic curve over a finite prime field", Math. USSR-Izv., 3:5 (1969), 1103-1114.

[8] И. М. Виноградов, "О распределении индексов", Докл. АН СССР, 4 (1926), 73-76.

[9] И. М. Виноградов, Основы теории чисел, 7-е изд., Наука, М., 1965, 172 с.

\footnotetext{
${ }^{8}$ Некоторые результаты работы [83] были улучшены Конягиным [84] на основе геометрической теории чисел.
} 
[10] M.Z. Garaev, "The sum-product estimate for large subsets of prime fields", Proc. Amer. Math. Soc., 136:8 (2008), 2735-2739.

[11] D. Hart, A. Iosevich, J. Solymosi, "Sum product estimates in finite fields via Kloosterman sums", Int. Math. Res. Not. IMRN, 2007:5, Art. ID rnm007, 14 pp.

[12] M. Z. Garaev, "An explicit sum-product estimate in $\mathbb{F}_{p}$ ", Int. Math. Res. Not. IMRN, 2007:11, Art. ID rnm035, 11 pp.; arXiv: math/0702780.

[13] S. V. Konyagin, I. E. Shparlinski, Character sums with exponential functions and their applications, Cambridge Tracts in Math., 136, Cambridge Univ. Press, Cambridge, 1999, ISBN: 0-521-64263-9, viii+163 pp.

[14] Н. М. Коробов, Тригонометрические суммы и их приложения, Наука, М., 1989, $240 \mathrm{c}$.

[15] G.H. Hardy, J.E. Littlewood, "A new solution of Waring's problem", Quart. J. Math. (2), 48 (1919), 272-293.

[16] A. Garcia, J. F. Voloch, "Fermat curves over finite fields", J. Number Theory, 30:3 (1988), 345-356.

[17] И.Е. Шпарлинский, "Об оценках сумм Гаусса", Матем. заметки, 50:1 (1991), 122-130; англ. пер.: I. E. Shparlinskii, "Estimates of Gaussian sums", Math. Notes, 50:1-2 (1991), 740-746.

[18] D. R. Heath-Brown, S. V. Konyagin, "New bounds for Gauss sums derived from $k$ th powers, and for Heilbronn's exponential sum", Q. J. Math., 51:2 (2000), 221-235.

[19] С. В. Конягин, "Оценки тригонометрических сумм по подгруппам и сумм Гаусса", Труды IV международной конференции "Современные проблемы теории чисел и ее приложения" (Тула, 2001), Изд-во МГУ, М., 2002, 86-114.

[20] J. Bourgain, "Multilinear exponential sums in prime fields under optimal entropy condition on the sources", Geom. Funct. Anal., 18:5 (2009), 1477-1502.

[21] А.А. Карацуба, "Об оценках полных тригонометрических сумм", Матем. заметки, 1:2 (1967), 199-208; англ. пер.: А. A. Karatsuba, "On estimates of complete trigonometric sums", Math. Notes, 1:2 (1967), 133-139.

[22] H.L. Montgomery, Turan’s method, Report № 15, Inst. Mittag-Leffler, Stockholm, 1978.

[23] S. M. Gonek, "A note on Turán's method", Michigan Math. J., 28:1 (1981), 83-87.

[24] H. L. Montgomery, R. C. Vaughan, T. D. Wooley, "Some remarks on Gauss sums associated with kth powers", Math. Proc. Cambridge Philos. Soc., 118:1 (1995), 21-33.

[25] S. V. Konyagin, "Good distribution of values of sparce polynomials modulo a prime", Analytic number theory, Cambridge Univ. Press, Cambridge, 2009, 289-296.

[26] A. A. Glibichuk, S. V. Konyagin, "Additive properties of product sets in fields of prime order", Additive combinatorics, CRM Proc. Lecture Notes, 43, Amer. Math. Soc., Providence, RI, 2007, 279-286.

[27] А.А. Глибичук, "Комбинаторные свойства множеств вычетов по простому модулю и задача Эрдёша-Грэхэма", Матем. заметки, 79:3 (2006), 384-395; англ. пер.: A. A. Glibichuk, "Combinational properties of sets of residues modulo a prime and the Erdös-Graham problem", Math. Notes, 79:3-4 (2006), 356-365.

[28] T. Tao, V. Vu, Additive combinatorics, Cambridge Stud. Adv. Math., 105, Cambridge Univ. Press, Cambridge, 2006, ISBN: 0-521-85386-9, xviii+512 pp.

[29] N.H. Katz, C.-Y. Shen, "A slight improvement to Garaev's sum product estimate", Proc. Amer. Math. Soc., 136:7 (2008), 2499-2504.

[30] N. H. Katz, C.-Y. Shen, "Garaev's inequality in finite fields not of prime order", Online J. Anal. Comb., 2008, № 3, Art. 3, 6 pp.

[31] J. Bourgain, M. Z. Garaev, "On a variant of sum-product estimates and explicit exponential sum bounds in prime fields", Math. Proc. Cambridge Philos. Soc., 146:1 (2009), 1-21. 
[32] I. Z. Ruzsa, "On the cardinality of $A+A$ and $A-A$ ", Combinatorics (Proc. Fifth Hungarian Colloq., Keszthely, 1976), Colloq. Math. Soc. János Bolyai, 18, II, North-Holland, Amsterdam-New York, 1978, 933-938.

[33] I. Z. Ruzsa, "An application of graph theory to additive number theory", Sci. Ser. A Math. Sci. (N. S.), 3 (1989), 97-109.

[34] M.B. Nathanson, Additive number theory. Inverse problems and the geometry of sumsets, Grad. Texts in Math., 165, Springer-Verlag, New York, 1996, ISBN: 0-387-94655-1, 293 pp.

[35] I. Z. Ruzsa, "Sums of finite sets", Number theory (New York, 1991-1995), Springer, New York, 1996, 281-293.

[36] A. Balog, E. Szemerédi, "A statistical theorem of set addition", Combinatorica, 14:3 (1994), 263-268.

[37] W.T. Gowers, "A new proof of Szemerédi's theorem", Geom. Funct. Anal., 11:3 (2001), 465-588.

[38] A. Balog, "Many additive quadruples", Additive combinatorics, CRM Proc. Lecture Notes, 43, Amer. Math. Soc., Providence, RI, 2007, 39-49.

[39] B. Sudakov, E. Szemerédi, V.H. Vu, "On a question of Erdös and Moser", Duke Math. J., 129:1 (2005), 129-155.

[40] M.Z. Garaev, "A quantified version of Bourgain's sum-product estimate in $\mathbb{F}_{p}$ for subsets of incomparable sizes", Electron. J. Combin., 15:1 (2008), Research paper 58, 8 pp.

[41] A. Weil, "On some exponential sums", Proc. Natl. Acad. Sci. USA, 34:5 (1948), 204-207.

[42] С. А. Степанов, Арифметика алгебраических кривых, Наука, M., 1991, ISBN: 5-02-014607-2, 368 с.; англ. пер.: S. A. Stepanov, Arithmetic of algebraic curves, Monogr. Contemp. Math., Consultants Bureau, New York, 1994, ISBN: 0-306-11036-9, xiv $+422 \mathrm{pp}$.

[43] L. J. Mordell, "On a sum analogous to Gauss's sum", Quart. J. Math., 3 (1932), 161-167.

[44] T. Cochrane, Ch. Pinner, "An improved Mordell type bound for exponential sums", Proc. Amer. Math. Soc., 133:2 (2005), 313-320.

[45] T. Cochrane, J. Coffelt, Ch. Pinner, "A further refinement of Mordell's bound on exponential sums", Acta Arith., 116:1 (2005), 35-41.

[46] J. Bourgain, "Mordell's exponential sum estimate revisited", J. Amer. Math. Soc., 18:2 (2005), 477-499.

[47] J. Bourgain, "Some arithmetical applications of the sum-product theorems in finite fields", Geometric aspects of functional analysis, Lecture Notes in Math., 1910, Springer, Berlin, 2007, 99-116.

[48] T. Tao, "The sum-product phenomenon in arbitrary rings", Contrib. Discrete Math., 4:2 (2009), 59-82.

[49] J. Bourgain, "Estimates on exponential sums related to the Diffie-Hellman distributions", Geom. Funct. Anal., 15:1 (2005), 1-34.

[50] R. Canetti, J. Friedlander, S. Konyagin, M. Larsen, D. Lieman, I. Shparlinski, "On the statistical properties of Diffie-Hellman distributions", Israel J. Math., 120 (2000), 23-46.

[51] R. Canetti, J. Friedlander, I. Shparlinski, "On certain exponential sums and the distribution of Diffie-Hellman triples", J. London Math. Soc. (2), 59:3 (1999), 799-812.

[52] J.B. Friedlander, S. Konyagin, I. E. Shparlinski, "Some doubly exponential sums over $\mathbb{Z}_{m} "$, Acta Arith., 105:4 (2002), 349-370.

[53] J. B. Friedlander, I. E. Shparlinski, "Double exponential sums over thin sets", Proc. Amer. Math. Soc., 129:6 (2001), 1617-1621. 
[54] M. Z. Garaev, "Double exponential sums related to Diffie-Hellman distributions", Int. Math. Res. Not., 2005:17 (2005), 1005-1014.

[55] M. Z. Garaev, A. A. Karatsuba, "New estimates of double trigonometric sums with exponential functions", Arch. Math. (Basel), 87:1 (2006), 33-40.

[56] M.-Ch. Chang, Ch. Zh. Yao, "An explicit bound on double exponential sums related to Diffie-Hellman distributions", SIAM J. Discrete Math., 22:1 (2008), 348-359.

[57] W. D. Banks, A. Conflitti, J. B. Friedlander, I. E. Shparlinski, "Exponential sums with Mersenne numbers", Compos. Math., 140:1 (2004), 15-30.

[58] M. Z. Garaev, "The large sieve inequality for the exponential sequence $\lambda^{\left[O\left(n^{15 / 14+o(1)}\right)\right]}$ modulo primes", Canad. J. Math., 61:2 (2009), 336-350.

[59] M. Z. Garaev, I. E. Shparlinski, "The large sieve inequality with exponential functions and the distribution of Mersenne numbers modulo primes", Int. Math. Res. Not., 2005:39 (2005), 2391-2408.

[60] J. Bourgain, M.-C. Chang, "Exponential sum estimates over subgroups and almost subgroups of $\mathbb{Z}_{Q}^{*}$, where $Q$ is composite with few prime factors", Geom. Funct. Anal., 16:2 (2006), 327-366.

[61] J. Bourgain, "The sum-product theorem in $\mathbb{Z}_{q}$ with $q$ arbitrary", J. Anal. Math., 106:1 (2008), 1-93.

[62] J. Bourgain, "Sum-product theorems and exponential sum bounds in residue classes for general modulus", C. R. Math. Acad. Sci. Paris, 344:6 (2007), 349-352.

[63] J. Bourgain, "Exponential sum estimates over subgroups of $\mathbb{Z}_{q}^{*}, q$ arbitrary", J. Anal. Math., 97:1 (2005), 317-355.

[64] E. Szemerédi, W.T. Trotter Jr., "Extremal problems in discrete geometry", Combinatorica, 3:3-4 (1983), 381-392.

[65] G. Elekes, "On the number of sums and products", Acta Arith., 81:4 (1997), 365-367.

[66] M. Z. Garaev, Ch.-Y. Shen, "On the size of the set $A(A+1)$ ", Math. Z., 265:1 (2010), 125-132.

[67] Le Anh Vinh, Szemerédi-Trotter type theorem and sum-product estimate in finite fields, arXiv: $0711.4427 \mathrm{v} 1$.

[68] J. Bourgain, "More on sum-product phenomenon in prime fields and its applications", Int. J. Number Theory, 1:1 (2005), 1-32.

[69] M.-Ch. Chang, "Some problems related to sum-product theorems", Additive combinatorics, CRM Proc. Lecture Notes, 43, Amer. Math. Soc., Providence, RI, 2007, 235-240.

[70] N. Hegyvari, F. Hennecart, "Explicit constructions of extractors and expanders", Acta Arith., 140:3 (2009), 233-249.

[71] D. Zuckerman, "Linear degree extractors and the inapproximability of max clique and chromatic number", Theory Comput., 3 (2007), 103-128.

[72] N. H. Katz, T. Tao, "Some connections between Falconer's distance set conjecture and sets of Furstenburg type", New York J. Math., 7 (2001), 149-187.

[73] B. Barak, R. Implagliazzo, A. Wigderson, "Extracting randomness using few independent sources", SIAM J. Comput., 36:4 (2006), 1095-1118.

[74] B. Barak, G. Kindler, R. Shaltiel, B. Sudakov, A. Wigderson, "Simulating independence: new constructions of condensers, Ramsey graphs, dispersers, and extractors", STOC'05: Proceedings of the 37th Annual ACM Symposium on Theory of Computing, ACM, New York, 2005, 1-10.

[75] J. Bourgain, "On the construction of affine extractors", Geom. Funct. Anal., 17:1 (2007), 33-57.

[76] H. A. Helfgott, "Growth and generation in $\mathrm{SL}_{2}(\mathbb{Z} / p \mathbb{Z})$ ", Ann. of Math. (2), 167:2 (2008), 601-623. 
[77] H. A. Helfgott, "Growth in $\mathrm{SL}_{3}(\mathbb{Z} / p \mathbb{Z})$ ", J. Eur. Math. Soc. (to appear); arXiv: 0807.2027.

[78] J. Bourgain, A. Gamburd, "New results on expanders", C. R. Math. Acad. Sci. Paris, 342:10 (2006), 717-721.

[79] J. Bourgain, A. Gamburd, "Uniform expansion bounds for Cayley graphs of $\mathrm{SL}_{2}\left(\mathbb{F}_{p}\right)$ ", Ann. of Math. (2), 167:2 (2008), 625-642.

[80] G. Davidoff, P. Sarnak, A. Valette, Elementary number theory, group theory, and Ramanujan graphs, London Math. Soc. Stud. Texts, 55, Cambridge Univ. Press, Cambridge, 2003, ISBN: 0-521-82426-5, x+144 pp.

[81] Sh. Hoory, N. Linial, A. Wigderson, "Expander graphs and their applications", Bull. Amer. Math. Soc. (N. S.), 43:4 (2006), 439-561.

[82] P. Sarnak, "What is .. an expander?", Notices Amer. Math. Soc., 51:7 (2004), $762-763$.

[83] M.-Ch. Chang, "On a question of Davenport and Lewis and new character sum bounds in finite fields", Duke Math. J., 145:3 (2008), 409-442.

[84] С. В. Конягин, "Оценки сумм характеров в конечных полях", Матем. заметки (в печати).

[85] M.-C. Chang, "Some problems in combinatorial number theory", Integers, 8:2 (2008), A1, 11 pp.

\section{3. Гараев (M. Z. Garaev)}

National Autonomous University of Mexico,

Institute of Mathematics

E-mail: garaev@matmor.unam.mx
Поступила в редакцию

14.05 .2009 